\title{
On The Representation Theory of $\mathrm{GL}\left(2, \mathbb{F}_{q}\right)$
}

\author{
by
}

\section{Gurjyot Kaur}

\begin{abstract}
A Thesis submitted to the Faculty of Graduate Studies of
The University of Manitoba

in partial fulfilment of the requirements of the degree of
\end{abstract}

MASTER OF SCIENCE

Department of Mathematics

University of Manitoba

Winnipeg, Canada

Copyright (c) 2020 by Gurjyot Kaur 


\section{Abstract}

Let $G=\mathrm{GL}\left(2, \mathbb{F}_{q}\right)$ denote the group of invertible $2 \times 2$ matrices over the finite field $\mathbb{F}_{q}$, where $q$ is the power of a prime number. This thesis investigates the complex irreducible representations of $G$. First, we give an overview of the general theory of finite-dimensional representations of a finite group over the complex numbers. Then we discuss the different types of conjugacy classes in $G$. It will turn out that there are four types, which altogether give $q^{2}-1$ conjugacy classes. By general representation theory, the number of distinct irreducible representations of $G$ is also equal to $q^{2}-1$. We proceed to construct these representations explicitly. Some of them are derived from the permutation representation of $G$ on the projective line $\mathbb{P}^{1}\left(\mathbb{F}_{q}\right)$, while the rest are induced from smaller subgroups of $G$. In the concluding chapter, we compute the irreducible decompositions of pairwise tensor products of these representations by analysing the inner products of their characters. These computations are an original contribution of this thesis. 


\section{Acknowledgments}

First of all thanks to almighty 'Guru Granth Sahib Ji' for His countless blessings, providing me the opportunity, and giving me the capability to complete my Master's thesis successfully. I would like to express my earnest thanks and incalculable respect to my supervisors Dr. Jaydeep Chipalkatti and Dr. Siddarth Sankaran whose sincere supervision has led me to this success. Their invaluable advice, learned guidance and timely suggestions helped me to bring my dissertation into final form and also lighted new ways to seek knowledge for me. Dr. Jaydeep gave me the motivation and the spirit to help me achieve my goals. Without his guidance, I could not have completed this research. I would like to thank Dr. Derek Krepski and Dr. Yang Zhang for serving on my thesis committee. I would also like to acknowledge the financial support given to me by my supervisors' NSERC grants and the Department of Mathematics.

I wish to extend my utmost thank to my dear father Late Mahender Singh Gandhi and my mother Sukhvinder Kaur, for their great role in my life and for believing in my ability to be successful in the every field. I would like to express my sincere thankfulness to my brother Atinder Singh, my sister-in-law Chanpreet Kaur, my little nephew Manchit Singh and my fiancé Guriqbal Singh. Without their prayers, it was indeed impossible to successfully complete this assignment. I would like to thank my family and friends for their love and care. 


\section{Contents}

1 Introduction

$1.1 \quad$ Group Representations $\ldots \ldots \ldots$. . . . . . . . . . . . . . 6

1.2 Outline of the Thesis $\ldots \ldots \ldots \ldots \ldots \ldots \ldots \ldots$

2 Representation Theory of Finite Groups 10

$2.1 \quad$ Representations $\ldots \ldots \ldots \ldots \ldots \ldots$

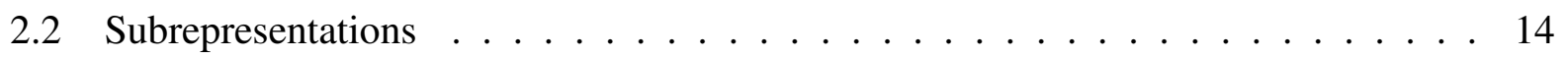

$2.3 \quad G$-equivariant Morphisms $\ldots \ldots \ldots \ldots \ldots \ldots$

2.4 Irreducible Representations $\ldots \ldots \ldots \ldots$

$2.5 \quad$ Irreducible Representations and Conjugacy Classes $\ldots \ldots \ldots$. . . . . . . 17

2.6 Character Theory for Finite Groups $\ldots \ldots \ldots$

2.7 The Character Table . . . . . . . . . . . . . . . . . . . . . . . 2 21

2.8 Tensor Product of Representations $\ldots \ldots \ldots \ldots$

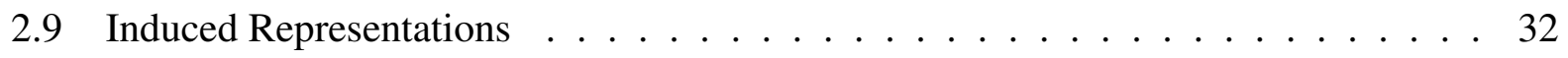

3 Conjugacy Classes in $\mathbf{G L}\left(2, \mathbb{F}_{q}\right)$

3.1 The $\operatorname{Order}$ of $G \ldots \ldots \ldots \ldots \ldots \ldots \ldots$

3.2 Eigenvalues $\ldots \ldots \ldots \ldots \ldots \ldots$

3.3 Stabilizers of Group Elements $\ldots \ldots \ldots \ldots$ 
4 The Irreducible Representations of $\mathbf{G L}\left(2, \mathbb{F}_{q}\right)$

4.1 The Permutation Representation $\ldots \ldots \ldots$. . . . . . . . . . . . 48

4.2 Character Values of Permutation Representation . . . . . . . . . . . . . . . 499

4.3 Complementary $q$-Dimensional Representation $\ldots \ldots \ldots \ldots$. . . . . . . . . .

4.4 One-dimensional Representations $\ldots \ldots \ldots \ldots \ldots$

4.5 The Representations $W_{\alpha, \beta} \quad \ldots \ldots \ldots \ldots \ldots \ldots \ldots$

4.6 The Representation $X_{\phi} \ldots \ldots \ldots \ldots \ldots$. . . . . . . . . . . . . . .

4.7 The Character Table of $\mathrm{GL}\left(2, \mathbb{F}_{q}\right) \quad \ldots \ldots \ldots \ldots \ldots$

5 Clebsch-Gordan Decompositions 72

5.1 Preliminary Results $\ldots \ldots \ldots \ldots \ldots \ldots \ldots \ldots$

5.2 Tensor Products with $U_{\alpha} \ldots \ldots \ldots \ldots \ldots \ldots \ldots \ldots$

5.3 The Decomposition of $V \otimes V] \ldots \ldots \ldots \ldots \ldots$

5.4 The Decomposition of $V \otimes W_{\alpha, 1} \ldots \ldots \ldots \ldots \ldots$

5.5 The Decomposition of $V \otimes X_{\phi} \ldots \ldots \ldots \ldots \ldots \ldots$

5.6 The Decomposition of $W_{\alpha, 1} \otimes X_{\phi} \ldots \ldots \ldots \ldots \ldots \ldots \ldots \ldots$

$5.7 \quad$ The Decomposition of $X_{\phi} \otimes X_{\psi} \ldots \ldots \ldots \ldots$

$5.8 \quad$ The Decomposition of $W_{\alpha, 1} \otimes W_{\beta, 1} \ldots \ldots \ldots \ldots \ldots \ldots \ldots$

Bibliography 


\section{Chapter 1}

\section{Introduction}

\subsection{Group Representations}

Representation theory is an important branch of mathematics. The beauty of the theory is that it relates two different algebraic structures, namely groups and vector spaces. The purpose of representation theory is to understand how a group can act on vector spaces. This thesis is concerned with finite-dimensional complex representations of a finite group, and specifically the group $\mathrm{GL}\left(2, \mathbb{F}_{q}\right)$ of nonsingular $2 \times 2$ matrices over the finite field $\mathbb{F}_{q}$.

Let $G$ be a finite group. A representation of $G$ is a complex vector space $V$ together with a homomorphism

$$
\rho: G \longrightarrow \mathrm{GL}(V) .
$$

In other words, for every $g \in G$ we have an invertible linear transformation $\rho_{g}: V \longrightarrow V$ such that

- $\rho_{g h}=\rho_{g} \circ \rho_{h}$ for any $g, h \in G$, and

- $\rho_{g^{-1}}=\rho_{g}^{-1}$ for any $g \in G$.

A subspace $W \subseteq V$ is called a subrepresentation if, for any $g \in G$, the map $\rho_{g}$ takes $W$ to $W$. The 
zero space $\{0\}$ and the whole space $V$ are always subrepresentations of $V$. A subrepresentation $W \subseteq V$ is called non-trivial if $W \neq\{0\}, V$.

If $V$ has a non-trivial subrepresentation then it is called reducible, otherwise it is called irreducible. For a finite group $G$, there are only finitely many irreducible representations up to isomorphism. They are the basic building blocks for all other representations in the sense that any finite-dimensional representation is a direct sum of irreducibles.

\subsection{Outline of the Thesis}

Let $G=\operatorname{GL}\left(2, \mathbb{F}_{q}\right)$ denote the group of invertible $2 \times 2$ matrices over the finite field $\mathbb{F}_{q}$, where $q$ is the power of a prime number. This thesis investigates the finite-dimensional irreducible representations of $G$ over the field of complex numbers.

\subsection{1}

Chapter 2 is devoted to the basics of representation theory. The topics include irreducible representations, tensor product of representations, character theory and the notion of an induced representation. We have explained these concepts by including several examples such as the Dihedral group $D_{8}$ and the Symmetric groups $S_{3}, S_{4}$. We have included some essential results such as Maschke's theorem as well as orthogonality of characters.

\subsection{2}

Chapter 3 describes the conjugacy classes in $G$. It turns out that there are altogether $q^{2}-1$ conjugacy classes, which divide into four types. Given a $2 \times 2$ matrix $A \in \mathrm{GL}\left(2, \mathbb{F}_{q}\right)$, its eigenvalues may lie in $\mathbb{F}_{q}$ or a quadratic extension of $\mathbb{F}_{q}$. In the former case, there may be two distinct eigenvalues or a single eigenvalue of multiplicity two. If the multiplicity is two, then $A$ is either diagonalizable 
or has a non-trivial Jordan canonical form. These divisions lead to the four types.

\subsection{3}

Chapter 4 describes all the irreducible representations of $G$. By general theory, we know that the number of irreducible representations is equal to the number of conjugacy classes. Hence $G$ must have $q^{2}-1$ mutually non-isomorphic irreducible representations.

- First, we start with the permutation representation of $G$ on the projective line $\mathbb{P}^{1}\left(\mathbb{F}_{q}\right)$. By its decomposition into irreducibles we get a $q$-dimensional irreducible representation $V$. Each character $\alpha: \mathbb{F}_{q}^{*} \longrightarrow \mathbb{C}^{*}$ determines a one-dimensional (irreducible) representation $U_{\alpha}$ of $G$. Then the tensor product of $V$ and $U_{\alpha}$ gives us another $q$-dimensional irreducible representation $V_{\alpha}$.

- Let $B \subseteq G$ be the Borel subgroup of upper triangular matrices, and let $N \subseteq B$ be the subgroup of unipotent upper triangular matrices. Then we have an isomorphism

$$
B / N \cong \mathbb{F}_{q}^{*} \times \mathbb{F}_{q}^{*}
$$

Given two distinct characters $\alpha, \beta$ of $\mathbb{F}_{q}^{*}$, the composite map

$$
B \rightarrow B / N \stackrel{\sim}{\longrightarrow} \mathbb{F}_{q}^{*} \times \mathbb{F}_{q}^{*} \stackrel{\alpha \times \beta}{\longrightarrow} \mathbb{C}^{*} \times \mathbb{C}^{*} \stackrel{\text { mult }}{\longrightarrow} \mathbb{C}^{*}
$$

gives a character $B \longrightarrow \mathbb{C}^{*}$. Let $W_{\alpha, \beta}$ denote the corresponding induced representation of $G$. It turns out to be irreducible. The distinct unordered pairs $\{\alpha, \beta\}$ give mutually nonisomorphic representations.

- Finally, we have a subgroup $K \subseteq \mathrm{GL}\left(2, \mathbb{F}_{q}\right)$ which is isomorphic to the multiplicative group $\mathbb{F}_{q^{2}}^{*}$. Consider a character $\phi:(K \simeq) \mathbb{F}_{q^{2}}^{*} \longrightarrow \mathbb{C}^{*}$ such that $\phi \neq \phi^{q}$, and let $Y_{\phi}$ be the 
induced representation of $G$. It turns out that $Y_{\phi}$ is reducible, but it gives rise to a new type of irreducible subrepresentation $X_{\phi}$. We have $X_{\phi} \cong X_{\phi^{q}}$, but two characters $\phi$ and $\psi$ of $\mathbb{F}_{q^{2}}^{*}$ such that $\phi \neq \psi, \psi^{q}$ give rise to non-isomorphic representations $X_{\phi}$ and $X_{\psi}$.

In summary, we get four types of irreducible representations of $G$, namely $U_{\alpha}, V_{\alpha}, W_{\alpha, \beta}$ and $X_{\phi}$. We also calculate a comprehensive character table for all of these representations.

\subsection{4}

If $E_{1}$ and $E_{2}$ are any two such irreducible representations, then their tensor product $E_{1} \otimes E_{2}$ will in general decompose into a direct sum of irreducibles. These are usually called Clebsch-Gordan decompositions, since in the 19th century Clebsch and Gordan studied them in the context of representations of the group $\mathrm{GL}(2, \mathbb{C})$ (see [9, Ch. 4]). In Chapter 5, we calculate the pairwise tensor product decompositions of these irreducible representations by using the character table. Altogether we carry out six different computations, namely the irreducible decompositions in the following cases:

$$
V \otimes V, \quad V \otimes W_{\alpha, 1}, \quad V \otimes X_{\phi}, \quad W_{\alpha, 1} \otimes X_{\phi}, \quad X_{\phi} \otimes X_{\psi}, \quad W_{\alpha, 1} \otimes W_{\beta, 1} .
$$

These computations are an original contribution of this thesis in the sense that we haven't found them in the existing literature. Given arbitrary irreducible representations $E_{1}$ and $E_{2}$, the decomposition of $E_{1} \otimes E_{2}$ can be inferred from these computations.

For the theory described in Chapters 2 through 4, we have broadly followed the treatment in the text 'Representation Theory, A First Course' by Fulton and Harris [4]. For basic representation theory, we have also referred to the texts by Alperin and Bell [1] as well as James and Liebeck [7]. We use the text by Gallian [5] as a standard reference for abstract algebra, and in particular the theory of finite fields. 


\section{Chapter 2}

\section{Representation Theory of Finite Groups}

In this chapter we will recall the basic representation theory of finite groups over the field $\mathbb{C}$ of complex numbers. Throughout this chapter, let $G$ denote a finite group. All the vector spaces will be finite-dimensional and defined over $\mathbb{C}$. If $V$ is such a vector space, then $\mathrm{GL}(V)$ denotes the group of invertible $\mathbb{C}$-linear maps $V \rightarrow V$. A good expository account of basic representation theory can be found in the paper [10] by Teleman. All the necessary preliminaries for linear algebra may be found in [6].

\subsection{Representations}

Definition 2.1. A representation of a group $G$ on a vector space $V$ is a homomorphism

$$
\rho: G \rightarrow \mathrm{GL}(V)
$$

In other words, defining a representation of $G$ on $V$ translates into assigning for each $g \in G$ an invertible linear map $\rho(g): V \rightarrow V$ such that

$$
\rho(e)=1_{V}, \quad \rho(g h)=\rho(g) \rho(h) \quad \text { and } \quad \rho\left(g^{-1}\right)=\rho(g)^{-1} .
$$


We will denote this representation by $(\rho, V)$, or just by $V$ if $\rho$ is understood from the context.

We enclose a few examples of representations.

Example 2.2. Let $V$ be a one-dimensional vector space. We can define $\rho(g)=1_{V}$ for every $g \in G$, where $1_{V}$ denotes the identity map $V \rightarrow V$. This is called the trivial representation.

Example 2.3. Let $G=C_{4}=\left\{e, g, g^{2}, g^{3}\right\}$ denote the cyclic group of order 4 . Let $V=\mathbb{C}^{2}$ be the 2-dimensional vector space over $\mathbb{C}$, so that $\mathrm{GL}(V)$ can be identified with the group $\mathrm{GL}(2, \mathbb{C})$ of invertible $2 \times 2$ matrices. Now define $\rho: G \rightarrow \mathrm{GL}(2, \mathbb{C})$ as follows:

$$
\rho(e)=\left(\begin{array}{ll}
1 & 0 \\
0 & 1
\end{array}\right), \rho(g)=\left(\begin{array}{cc}
0 & -1 \\
1 & 0
\end{array}\right), \rho\left(g^{2}\right)=\left(\begin{array}{cc}
-1 & 0 \\
0 & -1
\end{array}\right), \rho\left(g^{3}\right)=\left(\begin{array}{cc}
0 & 1 \\
-1 & 0
\end{array}\right) .
$$

We can easily see that $\rho$ satisfies the equations 2.1). So, we have defined a representation of the group $C_{4}$.

Two representations $(\rho, V)$ and $(\psi, W)$ are said to be isomorphic, if there is a vector space isomorphism $f: V \rightarrow W$ such that we have

$$
f \circ \rho(g)=\psi(g) \circ f
$$

for all $g \in G$.

\subsubsection{Representations of $D_{8}$}

Let $D_{8}$ denote the dihedral group with 8 elements, generated by the elements $r, s$ subject to the relations

$$
r^{4}=s^{2}=e, \quad s r=r^{3} s .
$$

Thus

$$
D_{8}=\left\{e, r, r^{2}, r^{3}, s, r s, r^{2} s, r^{3} s\right\}
$$


We will determine all one-dimensional representations up to isomorphism. Let $\rho: D_{8} \rightarrow \operatorname{GL}(\mathbb{C}) \simeq$ $\mathbb{C}^{*}$ be any one-dimensional representation.

We know that $\rho(r)^{4}=\rho\left(r^{4}\right)=\rho(e)=1$. Since $\rho$ is a homomorphism, we must have $\rho(r)=$ $\pm 1, \pm i$. Similarly $\rho(s)^{2}=\rho\left(s^{2}\right)=\rho(e)=1$, which gives $\rho(s)= \pm 1$. Moreover, $\rho(r s)=$ $\rho\left(s r^{-1}\right) \Longrightarrow \rho(r) \rho(s)=\rho(s) \rho(r)^{-1} \Longrightarrow \rho(r)^{2}=1$. Hence $\rho(r)= \pm 1$. Thus we get the following four possibilities:

$$
(\rho(r), \rho(s))=(1,1), \quad(1,-1), \quad(-1,1), \quad(-1,-1)
$$

Any of them defines the representation completely.

Finally we will give an example of a two-dimensional representation $\phi: D_{8} \rightarrow \operatorname{GL}(2, \mathbb{C})$. Define

$$
\phi(r)=\left(\begin{array}{cc}
i & 0 \\
0 & -i
\end{array}\right), \quad \text { and } \phi(s)=\left(\begin{array}{ll}
0 & 1 \\
1 & 0
\end{array}\right)
$$

We can easily verify that $\phi$ satisfies the equations in 2.1 , and hence $\left(\phi, \mathbb{C}^{2}\right)$ is a representation.

\subsubsection{The symmetric group $S_{3}$}

Consider the symmetric group $S_{3}$ on the set $\{1,2,3\}$. Let $g=(123)$, where the length 3 -cycle (123) is a permutation sending 1 to 2,2 to 3 and 3 to 1 . Let $r=(23)$. We can easily compute

$$
\begin{aligned}
g^{3} & =(123)(123)(123)=(123)(132)=e, \\
r^{2} & =(23)(23)=e, \\
g r g & =(123)(23)(123)=(123)(13)=(23)=r .
\end{aligned}
$$


Hence we have a presentation

$$
S_{3}=\{e,(12),(13),(23),(123),(132)\} \simeq\left\langle g, r \mid g^{3}=r^{2}=e, g r=r g^{-1}\right\rangle .
$$

\subsection{3}

Now we will define some representations of $S_{3}$.

The trivial representation $\sigma: S_{3} \rightarrow \mathrm{GL}(\mathbb{C}) \simeq \mathbb{C}^{*}$ is defined by letting $(\sigma(g), \sigma(r))=(1,1)$. The sign representation (or alternating representation) $\sigma: S_{3} \rightarrow \mathrm{GL}(\mathbb{C}) \simeq \mathbb{C}^{*}$ is defined by

$$
\sigma(a)= \begin{cases}1 & \text { if } a \text { is an even permutation } \\ -1 & \text { if } a \text { is an odd permutation }\end{cases}
$$

for $a \in S_{3}$.

We proceed to define the so-called 'standard' representation. Let $e_{1}, e_{2}, e_{3}$ be the standard basis of $\mathbb{C}^{3}$, and let $E \subseteq \mathbb{C}^{3}$ the one-dimensional subspace generated by the vector $e_{1}+e_{2}+e_{3}$. Define $W$ to be the 2-dimensional quotient vector space $\mathbb{C}^{3} / E$. Now the standard representation

$$
\sigma: S_{3} \rightarrow \mathrm{GL}(W)
$$

is defined by the recipe that $\sigma(g)$ will send $e_{i}+E$ to $e_{g(i)}+E$ for all $g \in S_{3}$.

For instance, let $g=(12) \in S_{3}$. Then $\sigma_{(12)}\left(e_{1}+E\right)=e_{2}+E$ and $\sigma_{(12)}\left(e_{2}+E\right)=e_{1}+E$. If we use $\left\{e_{1}+E, e_{2}+E\right\}$ as a basis of $W$, then $\sigma_{(12)}$ is given by the matrix $\left(\begin{array}{ll}0 & 1 \\ 1 & 0\end{array}\right)$.

Now take $g=(132) \in S_{3}$. Then $\sigma_{(132)}\left(e_{1}+E\right)=e_{3}+E=-e_{1}-e_{2}+E$ and $\sigma_{(132)}\left(e_{2}+E\right)=$ $e_{1}+E$. Therefore, $\sigma_{(132)}$ is given by the matrix $\left(\begin{array}{cc}-1 & 1 \\ -1 & 0\end{array}\right)$.

The representation theory of the groups $D_{8}$ and $S_{3}$ is also discussed in [2]. 


\subsection{Subrepresentations}

Let $\rho: G \rightarrow \mathrm{GL}(V)$ be a representation.

Definition 2.4. A subrepresentation of $V$ is a subspace $W \subseteq V$ with the property that we have $\rho(g)(w) \in W$ for all $w \in W$ and $g \in G$. Then $W$ itself becomes a representation of $G$. We also say that $W \subseteq V$ is a $G$-invariant subspace.

We will see some examples of subrepresentations.

Example 2.5. There are two trivial cases; namely the zero subspace $W=\{0\}$ and the whole space $W=V$ are always subrepresentations.

Example 2.6. Let $G=\mathbb{Z}$, where $\mathbb{Z}$ is a group under addition. Consider the two-dimensional representation $\phi: \mathbb{Z} \rightarrow \mathrm{GL}(2, \mathbb{C})$ given by

$$
\phi(n)=\left(\begin{array}{ll}
1 & n \\
0 & 1
\end{array}\right), \quad \text { for } n \in \mathbb{Z}
$$

Let $e_{1}=\left(\begin{array}{l}1 \\ 0\end{array}\right)$, and $V_{1}=\operatorname{span}\left\{e_{1}\right\} \subset \mathbb{C}^{2}$. We will show $V_{1}$ is $G$-invariant. If $v \in V_{1}$, then there exists $a \in \mathbb{C}$ such that $v=a e_{1}$. Now, $\phi(n)(v)=\left(\begin{array}{ll}1 & n \\ 0 & 1\end{array}\right)\left(\begin{array}{l}a \\ 0\end{array}\right)=\left(\begin{array}{l}a \\ 0\end{array}\right) \in V_{1}$. So, $V_{1}$ is $G$-invariant.

Now let $V_{2}=\operatorname{span}\left\{\left(\begin{array}{l}0 \\ 1\end{array}\right)\right\} \subset \mathbb{C}^{2}$. Since $\phi(1)\left(e_{2}\right) \notin V_{2}$, the subspace $V_{2}$ is not $G$-invariant. 


\section{3 $G$-equivariant Morphisms}

Definition 2.7. Let $\left(\rho_{1}, V_{1}\right)$ and $\left(\rho_{2}, V_{2}\right)$ be two representations of a group $G$. A map $\phi: V_{1} \rightarrow V_{2}$ is said to be morphism of representations if it is $G$-equivariant; that is to say, we have

$$
\rho_{2}(g)(\phi(v))=\phi\left(\rho_{1}(g)(v)\right)
$$

for all $v \in V_{1}$ and $g \in G$. In other words, the following diagram commutes:

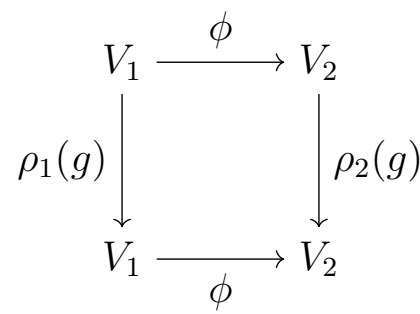

In particular, we say that the representations are isomorphic if there exists a vector space isomorphism $\phi: V_{1} \longrightarrow V_{2}$ which is $G$-equivariant.

Proposition 2.8. Let $\phi$ be a morphism of representations as above. Then

(i) $\operatorname{Ker}(\phi)$ is a subrepresentation of $V_{1}$.

(ii) $\operatorname{Im}(\phi)$ is a subrepresentation of $V_{2}$.

ProOF. (i) Let $v \in \operatorname{Ker}(\phi)$. Then $\phi(v)=0$. Since

$$
\phi\left(\rho_{1}(g)(v)\right)=\rho_{2}(g)(\phi(v))=0
$$

it follows that $\rho_{1}(g)(v) \in \operatorname{Ker}(\phi)$. Hence, $\operatorname{Ker}(\phi)$ is a subrepresentation of $V_{1}$.

(ii) Let $v \in \operatorname{Im}(\phi)$. Then there exist $v^{\prime} \in V_{1}$ such that $\phi\left(v^{\prime}\right)=v$. Now,

$$
\phi\left(\rho_{1}(g)\left(v^{\prime}\right)\right)=\rho_{2}(g)\left(\phi\left(v^{\prime}\right)\right)=\rho_{2}(g)(v)
$$


Therefore, $\rho_{2}(g)(v) \in \operatorname{Im}(\phi)$. Hence $\operatorname{Im}(\phi)$ is a subrepresentation of $V_{2}$.

\subsection{Irreducible Representations}

Definition 2.9. A representation $(\rho, V)$ of $G$ is called irreducible if it contains no non-trivial subrepresentations. That is to say, if $W \subset V$ is a subrepresentation, then either $W=\{0\}$ or $W=V$. Notice that all one-dimensional representations are irreducible.

Example 2.10. Recall the two-dimensional representation $\phi: D_{8} \rightarrow \mathrm{GL}(2, \mathbb{C})$ from Section 2.1.1 We will prove that $\phi$ is irreducible.

Suppose that $\phi$ is reducible. Then there must exist a one-dimensional subrepresentation $W \subset \mathbb{C}^{2}$. Assume that $W$ have a basis vector $\left(\begin{array}{l}a \\ b\end{array}\right)$. Now $\phi(r)\left(\begin{array}{l}a \\ b\end{array}\right)=\left(\begin{array}{cc}i & 0 \\ 0 & -i\end{array}\right)\left(\begin{array}{l}a \\ b\end{array}\right)=\left(\begin{array}{c}a i \\ -b i\end{array}\right)$ must be a scalar multiple of $\left(\begin{array}{l}a \\ b\end{array}\right)$, which is only possible if either $a=0$ or $b=0$.

Assume $a=0$, and hence $b \neq 0$. But then $\phi(s)\left(\begin{array}{l}0 \\ b\end{array}\right)=\left(\begin{array}{ll}0 & 1 \\ 1 & 0\end{array}\right)\left(\begin{array}{l}0 \\ b\end{array}\right)=\left(\begin{array}{l}b \\ 0\end{array}\right) \notin W$, which contradicts the property of $G$-invariance. We get a similar contraction if $b=0$. Hence $\phi$ must be irreducible.

Example 2.11. By the same approach as in the previous example, we will prove that the standard representation $\sigma: S_{3} \rightarrow G L(W)$ from Section 2.1.2 is irreducible.

Suppose that $\sigma$ is reducible, and assume that $U \subset W$ is a one-dimensional subrepresentation. Since $\sigma_{(12)}$ takes $U$ to $U$, it is easy to see that $q=e_{1}+e_{2}+E$ is a basis vector for $U$. But then $\sigma_{(123)}(q)=-e_{1}+E$ is not a scalar multiple of $q$, which is a contradiction. Hence $\sigma$ must be irreducible.

Lemma 2.12 (Schur's lemma). Let $(\rho, V)$ be an irreducible representation of $G$. Every morphism of representations $\phi: V \rightarrow V$ is a multiplication by scalar. 
Proof. Let $\lambda \in \mathbb{C}$ be an eigenvalue of $\phi$. Consider the eigenspace $E_{\lambda}=\{v \in V \mid \phi(v)=\lambda v\}$. First we will prove $E_{\lambda} \subset V$ is a subrepresentation of $V$. That is, we will show $E_{\lambda}$ is $G$-invariant. Let $v \in E_{\lambda}$. We claim that $\rho(g)(v) \in E_{\lambda}$. Consider,

$$
\begin{aligned}
\phi(\rho(g)(v)) & =\rho(g) \phi(v) \text { (Since } \phi \text { is } G \text {-equivariant) } \\
& =\rho(g)(\lambda v)=\lambda \rho(g)(v) .
\end{aligned}
$$

Therefore, $\rho(g)(v) \in E_{\lambda}$. This implies that $E_{\lambda}$ is a subrepresentation of $V$. Since $V$ is irreducible, we must have $E_{\lambda}=V$, i.e., $\phi(v)=\lambda v$ for all $v \in V$.

The following theorem shows that irreducible representations form 'building blocks' of all representations.

Theorem 2.13. (Maschke's Theorem) For any representation $V$ of a finite group $G$, there is a decomposition

$$
V=V_{1}^{\oplus a_{1}} \oplus V_{2}^{\oplus a_{2}} \oplus \ldots \oplus V_{k}^{\oplus a_{k}}
$$

where the $V_{i}$ are pairwise non-isomorphic irreducible representations. The decomposition of $V$ into a direct sum of the $k$ factors is unique, as are the $V_{i}$ that occur and their multiplicities $a_{i}$.

PROOF. The proof may be found in the standard text [4, Ch. 1].

\subsection{Irreducible Representations and Conjugacy Classes}

It is the case that a finite group $G$ admits only finitely many irreducible representations up to isomorphism. Moreover, it is a very useful fact that the number of such mutually non-isomorphic irreducible representations is equal to the number of conjugacy classes in $G$.

Definition 2.14. [Conjugacy Class] Two elements $a$ and $b$ in a group $G$ are said to be conjugate if there exists an element $g \in G$ such that $a=g b g^{-1}$. For $a \in G$, its conjugacy class is defined to 
the set

$$
C(a)=\left\{g a g^{-1} \mid g \in G\right\}
$$

These classes form a set partition of $G$.

Example 2.15. $D_{8}=\left\{e, r, r^{2}, r^{3}, s, r s, r^{2} s, r^{3} s\right\}$.

We will determine the conjugacy classes in $D_{8}$. We have

$$
\begin{gathered}
s^{-1} r s=s^{-1} s r^{-1}\left[\text { By using } r s=s r^{-1}\right] \\
=r^{-1}=r^{3}\left[\text { By using } r^{4}=e\right] \Longrightarrow r^{3} \in C(r) . \\
r^{-1} s r=r^{3} s r\left[\text { By using } r^{4}=e\right] \\
=r^{2} r s r=r^{2} s r^{3}\left[\text { By using } r s=s r^{-1}\right]=r^{2} s \Longrightarrow r^{2} s \in C(s) . \\
s^{-1} r s s=s^{-1} s r^{3} s\left[\text { By using } r s=s r^{-1}=s r^{3}\right] \\
=r^{3} s \Longrightarrow r^{3} s \in C(r s) .
\end{gathered}
$$

It is easy to check that there are no further conjugate pairs, and therefore $D_{8}$ has five conjugacy classes as follows: $\{e\},\left\{r, r^{3}\right\},\left\{s, r^{2} s\right\},\left\{r^{2}\right\},\left\{r s, r^{3} s\right\}$.

Theorem 2.16. Let $G$ be a finite group. Then the number of irreducible representations of $G$ is equal to the number of the conjugacy classes of $G$.

The proof may be found in the standard text [4, Ch. 2].

We have already seen five irreducible representations of $D_{8}$ in Section 2.1.1. The theorem tells us that any irreducible representation of $D_{8}$ is isomorphic to exactly one of the five.

Example 2.17. Consider the symmetric group

$$
S_{3}=\{e,(12),(13),(23),(123),(132)\}
$$


We proceed to compute the conjugacy classes of $S_{3}$.

$$
\begin{aligned}
& (12)(23)(12)=(12)(132)=(13) \quad \Longrightarrow(13) \in C((12)), \\
& (13)(12)(13)=(13)(132)=(23) \quad \Longrightarrow(23) \in C((12)), \\
& (12) 123)(12)=(12)(13)=(132) \quad \Longrightarrow(132) \in C((123)) .
\end{aligned}
$$

Therefore, $S_{3}$ has the following three conjugacy classes:

$$
\{e\}, \quad\{(12),(13),(23)\}, \quad\{(123),(132)\} \text {. }
$$

In Section 2.1.2, we have described three irreducible representations of $S_{3}$. As before, the theorem implies that any irreducible representation of $S_{3}$ must be isomorphic to exactly one of the three.

Proposition 2.18. Let $G$ be a finite group. Let $V_{1}, V_{2}, \ldots, V_{m}$ be the all the pairwise non-isomorphic irreducible representations of $G$ up to isomorphism. Then

$$
|G|=\sum_{i=1}^{m}\left(\operatorname{dim} V_{i}\right)^{2} .
$$

The proof may be found in [4, Ch. 2].

\subsection{Character Theory for Finite Groups}

In this section, we will discuss character theory for finite groups. This is an important tool in determining the irreducible representations of a group. A comprehensive discussion of character theory may be found in [7, 8]. 


\subsubsection{Characters}

Let $(\rho, V)$ be a representation of $G$. Then for each $g \in G$, we have a linear map $\rho(g): V \rightarrow V$ whose trace is well-defined.

Definition 2.19. The character of $(\rho, V)$ is defined as the complex valued function

$$
\chi_{V}: G \rightarrow \mathbb{C}, \quad \chi_{V}(g)=\operatorname{trace}(\rho(g)) .
$$

Example 2.20. We will calculate the characters of the standard representation $(\sigma, W)$ from Section 2.1.2. We have

$$
\begin{gathered}
\chi_{W}((12))=\operatorname{trace}(\sigma((12)))=\operatorname{trace}\left(\begin{array}{ll}
0 & 1 \\
1 & 0
\end{array}\right)=0 \\
\chi_{W}((132))=\operatorname{trace}(\sigma((132)))=\operatorname{trace}\left(\begin{array}{ll}
-1 & 1 \\
-1 & 0
\end{array}\right)=-1 .
\end{gathered}
$$

The following proposition collects together some basic results about characters. The proofs may be found in [4, Ch. 2].

Proposition 2.21. Let $(\rho, V)$ and $(\sigma, W)$ be representations of $G$. Then

(i) $\chi_{V}\left(h g h^{-1}\right)=\chi_{V}(g)$,

(ii) $\chi_{V}\left(1_{G}\right)=\operatorname{dim} V$,

(iii) $\chi_{V}\left(g^{-1}\right)=\overline{\chi_{V}(g)}$,

(iv) $\chi_{V \oplus W}(g)=\chi_{V}(g)+\chi_{W}(g)$. 


\subsubsection{The inner product of characters}

Let $(\rho, V)$ and $(\sigma, W)$ be representations of $G$. Their inner product is defined to be

$$
\left\langle\chi_{V}, \chi_{W}\right\rangle=\frac{1}{|G|} \sum_{g \in G} \chi_{V}(g) \overline{\chi_{W}(g)}
$$

We will mention several properties of characters which will be useful in the sequel. The proofs of all of these can be found in [4, Ch. 2]. In the next section, we will illustrate these properties using the examples of groups $D_{8}$ and $S_{3}$.

Proposition 2.22. Let $(\rho, V)$ and $(\sigma, W)$ be representations of $G$.

(i) If $V$ is irreducible then $\left\langle\chi_{V}, \chi_{V}\right\rangle=1$ that is, $\left\|\chi_{V}\right\|^{2}=1$.

(ii) If $V$ and $W$ are irreducible and non-isomorphic, then $\left\langle\chi_{V}, \chi_{W}\right\rangle=0$.

(iii) Two irreducible representations $V$ and $W$ are isomorphic, if and only if we have $\chi_{V}(g)=$ $\chi_{W}(g)$ for all $g \in G$.

Proposition 2.23. Let $W$ be a possibly reducible representation of $G$. Suppose we have an isomorphism of representations

$$
W \simeq \bigoplus_{i=1}^{r} V_{i}^{n_{i}}
$$

where $V_{i}$ is irreducible with multiplicity $n_{i}$ and $V_{1}, \ldots, V_{r}$ are pairwise non-isomorphic. Then $n_{i}=\left\langle\chi_{V_{i}}, \chi_{W}\right\rangle$.

\subsection{The Character Table}

Let $G$ be a finite group. Let $C_{1}, C_{2}, \ldots, C_{m}$ be the conjugacy classes in $G$ so that we have a set partition

$$
G=C_{1} \cup C_{2} \cup \cdots \cup C_{m}
$$


Let $V_{1}, V_{2}, \ldots, V_{m}$ denote all the irreducible representations of $G$ up to isomorphism. The character table of $G$ is an array in which rows are labelled by irreducible representations and columns are labelled by the conjugacy classes. The entry in row $i$ and column $j$ is $\chi_{V_{i}}\left(C_{j}\right)$.

\subsubsection{Orthogonality relations}

As mentioned earlier, if $\chi_{i}, \chi_{j}$ are two distinct irreducible characters, then

$$
\left\langle\chi_{i}, \chi_{j}\right\rangle=0
$$

Now $c_{i}, c_{j}$ denote two columns of the table, considered as vectors in $\mathbb{C}^{m}$. Then we have

$$
\left\langle c_{i}, c_{j}\right\rangle= \begin{cases}0 & \text { if } i \neq j \\ \frac{|G|}{\left|C_{i}\right|} & \text { if } i=j .\end{cases}
$$

Example 2.24. Recall Section 2.1.1. The dihedral group

$$
D_{8}=\left\{e, r, r^{2}, r^{3}, s, r s, r^{2} s, r^{3} s\right\},
$$

has five conjugacy classes:

$$
\{e\},\left\{r, r^{3}\right\},\left\{s, r^{2} s\right\},\left\{r^{2}\right\},\left\{r s, r^{3} s\right\} .
$$

Recall that there are four one-dimensional representations $\rho: D_{8} \rightarrow \mathbb{C}^{*}$ corresponding to the choices

$$
(\rho(r), \rho(s))=(1,1), \quad(1,-1), \quad(-1,1), \quad(-1,-1) .
$$

Let $V_{1}, V_{2}, V_{3}, V_{4}$ denote these representations respectively. Let $V_{5}$ be the two-dimensional repre- 
sentation space corresponding to $(\phi(r), \phi(s))=\left(\left(\begin{array}{cc}i & 0 \\ 0 & -i\end{array}\right),\left(\begin{array}{ll}0 & 1 \\ 1 & 0\end{array}\right)\right)$.

We can easily compute that

$$
\chi_{V_{5}}(e)=2, \quad \chi_{V_{5}}(r)=0, \quad \chi_{V_{5}}(s)=0, \quad \chi_{V_{5}}\left(r^{2}\right)=-2, \quad \text { and } \quad \chi_{V_{5}}(r s)=0 .
$$

Therefore, the character table of $D_{8}$ is as follows:

\begin{tabular}{|r||r|r|r|r|r|}
\hline & $\{e\}$ & $\left\{r, r^{3}\right\}$ & $\left\{s, r^{2} s\right\}$ & $\left\{r^{2}\right\}$ & $\left\{r s, r^{3} s\right\}$ \\
\hline \hline$\chi_{V_{1}}$ & 1 & 1 & 1 & 1 & 1 \\
\hline$\chi_{V_{2}}$ & 1 & 1 & -1 & 1 & -1 \\
\hline$\chi_{V_{3}}$ & 1 & -1 & 1 & 1 & -1 \\
\hline$\chi_{V_{4}}$ & 1 & -1 & -1 & 1 & 1 \\
\hline$\chi_{V_{5}}$ & 2 & 0 & 0 & -2 & 0 \\
\hline
\end{tabular}

The reader should also see [7, Ch. II, $\S 9$ ] for a discussion of the representation theory of $D_{8}$. Now we can verify the orthogonality relations from Section 2.7.1. For instance, we have

$$
\left\langle\chi_{V_{1}}, \chi_{V_{5}}\right\rangle=\frac{1}{8}[1(1.2)+2(1.0)+2(1.0)+1(1 .(-2))+2(1.0)]=0 .
$$

Similarly, we can check that $\left\langle\chi_{V_{i}}, \chi_{V_{j}}\right\rangle=0$ for all $i, j \in\{1,2,3,4,5\}$ where $i \neq j$. On the other hand,

$$
\left\|\chi_{V_{5}}\right\|^{2}=\frac{1}{|G|} \sum_{g \in G} \overline{\chi_{V_{5}}(g)} \chi_{V_{5}}(g)=\frac{1}{8}[1(2.2)+2(0.0)+2(0.0)+1((-2) \cdot(-2))+2(0.0)]=1 .
$$


Similarly, we can check that $\left\|\chi_{V_{i}}\right\|^{2}=\left\langle\chi_{V_{i}}, \chi_{V_{i}}\right\rangle=1$ for all $i \in\{1,2,3,4,5\}$. Notice that

$$
\left|D_{8}\right|=8=\left(\operatorname{dim} V_{1}\right)^{2}+\left(\operatorname{dim} V_{2}\right)^{2}+\left(\operatorname{dim} V_{3}\right)^{2}+\left(\operatorname{dim} V_{4}\right)^{2}+\left(\operatorname{dim} V_{5}\right)^{2}=1+1+1+1+4
$$

Let $c_{1}, \ldots, c_{5}$ denote the columns of the character table. Then it is easy to see that the inner product of any two distinct columns is zero. On the other hand, for instance

$$
\left\|c_{3}\right\|^{2}=1^{2}+1^{2}+1^{2}+1^{2}=4=\frac{|G|}{\left|C_{3}\right|}=\frac{8}{2} .
$$

Example 2.25. Recall Example 2.1.2, where we have described the irreducible representations of the symmetric group

$$
S_{3} \simeq\left\langle g, r \mid g^{3}=r^{2}=e, g r=r g^{-1}\right\rangle .
$$

of order 6. Its conjugacy classes are

$$
\{e\}, \quad\{(12),(13),(23)\}, \quad\{(123),(132)\} .
$$

Let $V_{1}, V_{2}, V_{3}$ denote the representation spaces for the trivial, sign and standard representation respectively. Thus $\operatorname{dim} V_{1}=\operatorname{dim} V_{2}=1$ and $\operatorname{dim} V_{3}=2$. From the corresponding matrix descriptions it is easy to compute that

$$
\chi_{V_{2}}(e)=1, \quad \chi_{V_{2}}(12)=-1, \quad \chi_{V_{2}}(123)=1, \quad \chi_{V_{3}}(e)=2, \quad \chi_{V_{3}}(12)=0, \quad \chi_{V_{3}}(123)=-1 .
$$


Therefore, the character table of $S_{3}$ is

\begin{tabular}{|l||r|r|r|}
\hline & $\{e\}$ & $\{(12),(13),(23)\}$ & $\{(123),(132)\}$ \\
\hline \hline$\chi_{V_{1}}$ & 1 & 1 & 1 \\
\hline$\chi_{V_{2}}$ & 1 & -1 & 1 \\
\hline$\chi_{V_{3}}$ & 2 & 0 & -1 \\
\hline
\end{tabular}

Let us verify some properties from Section 2.6.2 on this example. We have

$$
\left\langle\chi_{V_{1}}, \chi_{V_{2}}\right\rangle=\frac{1}{|G|} \sum_{g \in G} \overline{\chi_{V_{1}}(g)} \chi_{V_{2}}(g)=\frac{1}{6}[1(1.1)+3(1 .(-1))+2(1.1)]=0,
$$

and similarly

$$
\left\langle\chi_{V_{1}}, \chi_{V_{3}}\right\rangle=\left\langle\chi_{V_{2}}, \chi_{V_{3}}\right\rangle=0
$$

The self inner products are

$$
\left\|\chi_{V_{1}}\right\|^{2}=\left\langle\chi_{V_{1}}(g), \chi_{V_{1}}(g)\right\rangle=\frac{1}{|G|} \sum_{g \in G} \overline{\chi_{V_{1}}(g)} \chi_{V_{1}}(g)=\frac{1}{6}[1(1.1)+3(1.1)+2(1.1)]=1,
$$

and similarly

$$
\left\|\left.\chi_{V_{2}}\right|^{2}=\right\| \chi_{V_{3}} \|^{2}=1
$$

Moreover,

$$
\left|S_{3}\right|=6=\left(\operatorname{dim} V_{1}\right)^{2}+\left(\operatorname{dim} V_{2}\right)^{2}+\left(\operatorname{dim} V_{3}\right)^{2}=1^{2}+1^{2}+2^{2} .
$$

\subsubsection{The symmetric group $S_{4}$}

Consider the symmetric group $S_{4}$ of order 24 . In this section we will determine the character table for $S_{4}$ using the properties in Section 2.6.2.

Let $V_{1}$ be the trivial representation of $S_{4}$, and $V_{2}$ the sign representation defined as in Section 2.1.2 
on page 12. Let $e_{1}, \ldots, e_{4}$ be the standard basis of $\mathbb{C}^{4}$, and $E \subseteq \mathbb{C}^{4}$ the subspace generated by the vector $e_{1}+\cdots+e_{4}$. Let $V_{3}=\mathbb{C}^{4} / E$ with the standard representation $\sigma: S_{4} \rightarrow \operatorname{GL}\left(V_{3}\right)$ defined as in Section 2.1.2.

By the general theory of symmetric groups (see [3, Ch. 1]), we know that two permutations in $S_{4}$ are conjugate if and only if they have the same cycle type. Therefore, each cycle type of $S_{4}$ corresponds to a conjugacy class. Following are the five conjugacy classes:

$$
\begin{aligned}
& \{e\}, \quad\{(12),(13),(14),(23),(24),(34)\}, \\
& \{(123),(132),(142),(124),(234),(243),(134),(143)\}, \\
& \{(1234),(1432),(1342),(1423),(1324),(1243)\}, \quad\{(12)(34),(13)(24),(14)(23)\} .
\end{aligned}
$$

We will calculate the character values $\chi_{V_{3}}(g)$ for one $g$ in each conjugacy class. It is clear that $\rho_{e}$ corresponds to the matrix $\rho_{e}=\left(\begin{array}{ccc}1 & 0 & 0 \\ 0 & 1 & 0 \\ 0 & 0 & 1\end{array}\right)$, and therefore $\chi_{V_{3}}(e)=3$.

For $g=(12)$, we have

$$
\rho_{g}\left(e_{1}+E\right)=e_{2}+E, \quad \rho_{g}\left(e_{2}+E\right)=e_{1}+E, \quad \rho_{g}\left(e_{3}+E\right)=e_{3}+E,
$$

which gives $\rho_{(12)}=\left(\begin{array}{ccc}0 & 1 & 0 \\ 1 & 0 & 0 \\ 0 & 0 & 1\end{array}\right)$. Hence $\chi_{V_{3}}(12)=1$.

For $g=(123)$, we have

$$
\rho_{g}\left(e_{1}+E\right)=e_{2}+E, \quad \rho_{g}\left(e_{2}+E\right)=e_{3}+E, \quad \rho_{g}\left(e_{3}+E\right)=e_{1}+E,
$$


which gives $\rho_{(123)}=\left(\begin{array}{lll}0 & 0 & 1 \\ 1 & 0 & 0 \\ 0 & 1 & 0\end{array}\right)$ and hence $\chi_{V_{3}}((123)=0$.

For $g=(1234)$, we have

$\rho_{g}\left(e_{1}+E\right)=e_{2}+E, \quad \rho_{g}\left(e_{2}+E\right)=e_{3}+E, \quad \rho_{g}\left(e_{3}+E\right)=e_{4}+E=-e_{1}-e_{2}-e_{3}+E$.

This gives $\rho_{(1234)}=\left(\begin{array}{ccc}0 & 0 & -1 \\ 1 & 0 & -1 \\ 0 & 1 & -1\end{array}\right)$, and hence $\chi_{V_{3}}(1234)=-1$.

For $g=(12)(34)$, we have

$\rho_{g}\left(e_{1}+E\right)=e_{2}+E, \quad \rho_{g}\left(e_{2}+E\right)=e_{1}+E, \quad \rho_{g}\left(e_{3}+E\right)=e_{4}+E=-e_{1}-e_{2}-e_{3}+E$.

This gives $\rho_{((12)(34))}=\left(\begin{array}{ccc}0 & 1 & -1 \\ 1 & 0 & -1 \\ 0 & 0 & -1\end{array}\right)$, and therefore $\chi_{V_{3}}((12)(34))=-1$. It is easy to check that $\left\|\chi_{V_{3}}\right\|^{2}=1$, and hence $V_{3}$ is irreducible.

\subsection{3}

We know that the number of irreducible representations is equal to the number of conjugacy classes. Therefore $S_{4}$ must have two other irreducible representations. Let $V_{4}$ and $V_{5}$ denote the other two irreducible representations of $S_{4}$. By Proposition 2.18, we have $|G|=\sum_{i}\left(\operatorname{dim} V_{i}\right)^{2}$. Therefore,

$$
24=\left|S_{4}\right|=1+1+9+\left(\operatorname{dim} V_{4}\right)^{2}+\left(\operatorname{dim} V_{5}\right)^{2},
$$


which implies that $\left(\operatorname{dim} V_{4}\right)^{2}+\left(\operatorname{dim} V_{5}\right)^{2}=13$. Hence we may assume that

$$
\operatorname{dim} V_{4}=3, \quad \text { and } \quad \operatorname{dim} V_{5}=2 .
$$

Our next step is to determine the character values of $V_{4}$ and $V_{5}$. At this point, we can write the character table is as follows :

\begin{tabular}{|r||r|r|r|r|r|}
\hline & $\{\mathrm{e}\}$ & $\{(12)\}$ & $\{(123)\}$ & $\{(1234)\}$ & $\{(12)(34)\}$ \\
\hline \hline$\chi_{V_{1}}$ & 1 & 1 & 1 & 1 & 1 \\
\hline$\chi_{V_{2}}$ & 1 & -1 & 1 & -1 & 1 \\
\hline$\chi_{V_{3}}$ & 3 & 1 & 0 & -1 & -1 \\
\hline$\chi_{V_{4}}$ & 3 & $a$ & $b$ & $c$ & $d$ \\
\hline$\chi_{V_{5}}$ & 2 & $u$ & $v$ & $w$ & $x$ \\
\hline
\end{tabular}

Here $a, b, c, d, u, v, w, x$ stand for the unknown values which we will try to determine.

\subsection{4}

Let $C_{1}, \ldots, C_{5}$ denote the conjugacy classes. They have $1,6,8,6,3$ elements respectively. We will use the orthogonality relations from Section 2.7.1. Taking the inner product of columns $c_{1}$ and $c_{2}$, we get $1-1+3+3 a+2 u=0$, i.e.,

$$
3 a+2 u=-3
$$

Using the fact that $\left\|c_{2}\right\|^{2}=\frac{|G|}{\left|C_{2}\right|}=\frac{24}{6}=4$, we get

$$
a^{2}+u^{2}=1
$$


These equations have the following two possible solutions:

$$
(a, u)=(-1,0) \quad \text { or } \quad(-5 / 13,-12 / 13) \text {. }
$$

Similarly, using columns $c_{1}$ and $c_{5}$, we get the equations

$$
d^{2}+x^{2}=5, \quad 3 d+2 x=1 .
$$

These have possible solutions

$$
(d, x)=(-1,2), \quad \text { or } \quad(19 / 13,-22 / 13) .
$$

But now, using $c_{2}$ and $c_{5}$ we have $a d+u x=1$, and hence the only possibility is

$$
(a, u)=(-1,0), \quad \text { and } \quad(d, x)=(-1,2) .
$$

Using similar calculations of columns $c_{1}, c_{2}, c_{3}$ we get $(b, v)=(0,-1)$, and using $c_{1}, c_{2}, c_{4}$ we get $(c, w)=(1,0)$. Hence, the character table of $S_{4}$ is fully determined as follows:

\begin{tabular}{|r||r|r|r|r|r|}
\hline & $\{\mathrm{e}\}$ & $\{(12)\}$ & $\{(123)\}$ & $\{(1234)\}$ & $\{(12)(34)\}$ \\
\hline \hline$\chi_{V_{1}}$ & 1 & 1 & 1 & 1 & 1 \\
\hline$\chi_{V_{2}}$ & 1 & -1 & 1 & -1 & 1 \\
\hline$\chi_{V_{3}}$ & 3 & 1 & 0 & -1 & -1 \\
\hline$\chi_{V_{4}}$ & 3 & -1 & 0 & 1 & -1 \\
\hline$\chi_{V_{5}}$ & 2 & 0 & -1 & 0 & 2 \\
\hline
\end{tabular}

The general theory of irreducible representations for the symmetric group $S_{n}$ may be found in [4. Ch. 4]. In particular, the representation spaces $V_{i}$ can be concretely described in terms of 
Young tableaux corresponding to the partitions of $n$.

\subsection{Tensor Product of Representations}

In this section, we will discuss the tensor product of two representations of a group $G$. For the definition of the tensor product of two vector spaces, see [3, Section 11.5] and [2, Ch. II, §12].

Definition 2.26. Let $(\rho, V)$ and $(\sigma, W)$ be representations of $G$. Their tensor product representation $(\rho \otimes \sigma, V \otimes W)$ is defined on pure tensors by

$$
(\rho \otimes \sigma)(g)(v \otimes w)=\rho(g)(v) \otimes \sigma(g)(w),
$$

for $g \in G$, and then extended by linearity. It is easy to see that this construction preserves equations (2.1) on page 10 .

The following examples will clarify this definition.

Example 2.27. In Example 2.3, we have defined a representation $\rho: C_{4} \rightarrow \operatorname{GL}(V)$ via the matrix $\rho(g)=\left(\begin{array}{cc}0 & -1 \\ 1 & 0\end{array}\right)$, where $g$ is a cyclic generator of $C_{4}$. Recall that $V=\mathbb{C}^{2}$ is two-dimensional vector space with standard basis $\left\{e_{1}, e_{2}\right\}$. Therefore, $V \otimes V$ has basis

$$
\left\{e_{1} \otimes e_{1}, e_{1} \otimes e_{2}, e_{2} \otimes e_{1}, e_{2} \otimes e_{2}\right\}
$$

Notice that $\rho(g)$ sends $e_{1}, e_{2}$ respectively to $e_{2},-e_{1}$. Now we have

$$
\begin{aligned}
& (\rho \otimes \rho)(g)\left(e_{1} \otimes e_{1}\right)=\rho(g)\left(e_{1}\right) \otimes \rho(g)\left(e_{1}\right)=e_{2} \otimes e_{2}, \\
& (\rho \otimes \rho)(g)\left(e_{1} \otimes e_{2}\right)=\rho(g)\left(e_{1}\right) \otimes \rho(g)\left(e_{2}\right)=e_{2} \otimes\left(-e_{1}\right)=-\left(e_{2} \otimes e_{1}\right) .
\end{aligned}
$$


Similarly, $(\rho \otimes \rho)(g)\left(e_{2} \otimes e_{2}\right)=e_{1} \otimes e_{1}$ and $(\rho \otimes \rho)(g)\left(e_{2} \otimes e_{1}\right)=-\left(e_{1} \otimes e_{2}\right)$.

Therefore, $(\rho \otimes \rho, V \otimes V)$ is defined via the matrix $\left(\begin{array}{cccc}0 & 0 & 0 & 1 \\ 0 & 0 & -1 & 0 \\ 0 & -1 & 0 & 0 \\ 1 & 0 & 0 & 0\end{array}\right)$ which specifies the action of $(\rho \otimes \rho)(g)$.

Definition 2.28. Let $A=\left[a_{i j}\right]$ and $B=\left[b_{k l}\right]$ be two matrices of size $m \times n$ and $r \times s$, respectively. The Kronecker product of $A$ and $B$ is defined as $A \otimes B=\left(\begin{array}{cccc}a_{11} B & a_{12} B & \cdots & a_{1 n} B \\ a_{21} B & a_{22} B & \cdots & a_{2 n} B \\ \vdots & \vdots & \vdots & \vdots \\ a_{m 1} B & a_{m 2} B & \cdots & a_{m n} B\end{array}\right)=\left[a_{i j} B\right]$. Notice that the size of the new matrix is $m r \times n s$. Also, $\operatorname{trace}(A \otimes B)=\operatorname{trace}(A) \operatorname{trace}(B)$.

If the matrices for $\rho(g)$ and $\sigma(g)$ are given, then the matrix for $(\rho \otimes \sigma)(g)$ is given by the Kronecker product of the two matrices. This implies the formula

$$
\chi_{V \otimes W}(g)=\chi_{V}(g) \chi_{W}(g)
$$

for any $g \in G$.

\subsubsection{The Dual Representation}

Let $V$ be a $G$-representation, corresponding to the map $\rho: G \longrightarrow \operatorname{GL}(V)$. Then the dual vector space $V^{\vee}=\operatorname{Hom}(V, \mathbb{C})$ is also $G$-representation in a natural way (see [4, Ch. 1]). The corresponding map

$$
\rho^{\vee}: G \longrightarrow \mathrm{GL}\left(V^{\vee}\right)
$$


takes the functional $\varphi \in V^{\vee}$ to the functional

$$
V \longrightarrow \mathbb{C}, \quad v \longrightarrow \varphi\left(\rho_{g^{-1}}(v)\right)
$$

If $\rho_{g}$ is given by the matrix $A$ with respect to a basis of $V$, then $\rho_{g}^{\vee}$ is given by $\left(A^{-1}\right)^{T}$ with respect to the dual basis. Hence we have

$$
\chi_{V} \vee(g)=\operatorname{trace}\left(\rho_{g}^{\vee}\right)=\chi_{V}\left(g^{-1}\right)=\overline{\chi_{V}(g)} .
$$

\subsection{Induced Representations}

Let $G$ be a finite group. Let $H$ be a subgroup of $G$. In this section we will discuss an important construction called 'induced representation'. Given a representation of $(\rho, W)$ of $H$, it gives a representation $(\psi, V)$ of the whole group $G$. We use the following steps:

- For every $h \in H$, we have a linear map

$$
\rho_{h}: W \longrightarrow W
$$

- Let $G / H$ be the set of left cosets of $H$ in $G$. For every coset $\sigma \in G / H$, choose a representative $g_{\sigma} \in \sigma$. Then $\sigma=g_{\sigma} H$. We have a set partition

$$
G=\bigcup_{\sigma \in G / H} g_{\sigma} H
$$

- Given an element $z \in G$, the element $z . g_{\sigma} \in G$ belongs to a unique left coset $\sigma_{z}$. Then we have a unique element $h_{z} \in H$ such that

$$
z \cdot g_{\sigma}=g_{\sigma_{z}} \cdot h_{z}
$$


- Take a copy $W^{\sigma}$ of $W$ for each left coset $\sigma \in G / H$. For $w \in W$, let $g_{\sigma} w$ denote the element of $W^{\sigma}$ corresponding to $w \in W$. Define the vector space

$$
V=\bigoplus_{\sigma \in G / H} W^{\sigma}
$$

so that every element of $V$ has a unique expression $v=\sum g_{\sigma} w_{\sigma}$ for some elements $w_{\sigma} \in W$.

- For $z \in G$, define a map

$$
\psi_{z}: V \longrightarrow V
$$

by the formula

$$
\psi_{z}\left(\sum g_{\sigma} w_{\sigma}\right)=\sum g_{\sigma_{z}} \rho_{h_{z}}\left(w_{\sigma}\right)
$$

This makes $V$ into a representation of $G$. The pair $(\psi, V)$ is called the representation induced from $(\rho, W)$. Sometimes we write $V=\operatorname{Ind}_{H}^{G} W$ if $\rho$ is understood.

Let $n=[G: H]$ be the index of $H$ in $G$. Then

$$
\operatorname{dim}(V)=n \cdot \operatorname{dim}(W)
$$

Example 2.29. Consider the symmetric group

$$
G=S_{3}=\{e,(12),(13),(23),(123),(132)\}
$$

Consider the subgroup $H=\{e,(12)\}$. Then $[G: H]=3$. It is easy to see that we have a coset decomposition

$$
G=H \cup(13) H \cup(23) H
$$

Let $\sigma_{1}, \sigma_{2}, \sigma_{3}$ denote these cosets respectively. Let $W=\langle w\rangle$ be a one-dimensional vector space, 
and consider the representation

$$
\rho: H \rightarrow \mathrm{GL}(W) \simeq \mathbb{C}^{*}
$$

such that $\rho(e)=1$ and $\rho((12))=-1$. In other words, $\rho_{e}(w)=w$ and $\rho_{(12)}(w)=-w$.

By the definition of the induced representation,

$$
V=W^{\sigma_{1}} \oplus W^{\sigma_{2}} \oplus W^{\sigma_{3}}
$$

Hence $\{w,(13) w,(23) w\}$ is a basis of $V$.

First, we will see an example of how $(12)$ acts on $V$. We have $(12)(13)=(132)=(23)(12)$, hence

$$
\psi_{(12)}((13) w)=(23) \rho_{(12)}(w)=-(23) w
$$

Using similar calculations, we see that with respect to the given basis of $V$, the action of (12) is represented by the matrix $\left(\begin{array}{ccc}-1 & 0 & 0 \\ 0 & 0 & -1 \\ 0 & -1 & 0\end{array}\right)$. Similarly, we can see that the action of $(123)$ following character table of $V$ :

\begin{tabular}{|c||c|c|c|}
\hline & $e$ & $\{(12),(13),(23)\}$ & $\{(123),(132)\}$ \\
\hline \hline$\chi_{V}$ & 3 & -1 & 0 \\
\hline
\end{tabular}

Then, $\left\|\chi_{V}\right\|^{2}=\frac{1}{6}\left(3^{2}+3(-1)(-1)\right)=2$. Hence $V$ is reducible. Comparing with the table in Example 2.25, we see that $V \simeq V_{2} \oplus V_{3}$. 


\subsubsection{The Character of an Induced Representation}

As before, let $W$ be a representation of $H$, and $V$ the induced representation of $G$. In this section, we will give a formula which gives the character of $V$ in terms of the character of $W$.

For $g \in G$, let $C_{G}(g)$ and $C_{H}(g)$ denote its conjugacy classes in $G, H$ respectively. In general, $C_{G}(g) \cap H$ will be a disjoint union of $H$-conjugacy classes

$$
C_{G}(g) \cap H=\bigcup_{i=1}^{m} C_{H}\left(x_{i}\right),
$$

where $x_{i} \in H$ are the representatives of distinct $H$-conjugacy class. If $C_{G}(g) \cap H=\emptyset$, then $m$ is understood to be zero. Recall that, for $g \in G$ we have the stabilizer subgroup

$$
G_{g}=\left\{s \in G: s^{-1} g s=g\right\} .
$$

Proposition 2.30. If $m=0$, then $\chi_{V}(g)=0$. Otherwise,

$$
\chi_{V}(g)=\left|G_{g}\right| \sum_{i=1}^{m} \frac{\chi_{W}\left(x_{i}\right)}{\left|H_{x_{i}}\right|} .
$$

A proof of this formula may be found in [1, Chapter 6].

Example 2.31. Let $G=S_{4}$ be the symmetric group and let

$$
H=\langle(1234)\rangle=\{e,(1234),(13)(24),(1432)\} .
$$

Since $H$ is abelian, every conjugacy class in $H$ is singleton.

Consider the group homomorphism $H \longrightarrow \mathbb{C}^{*}$ which sends $(1234)$ to $\iota$. This gives a onedimensional representation $W$ of $H$. Then the induced representation $V$ of $G$ has dimension $\left[S_{4}: H\right]=6$.

We already know that $\chi_{V}(e)=6$. If $g=(12)$ or $(123)$, then $C_{G}(g) \cap H=\emptyset$, and then $\chi_{V}(g)=0$. 
If $g=(13)(24)$, then $C_{G}(g) \cap H=\{(13)(24)\}$. In that case,

$$
\chi_{V}(g)=\left|G_{g}\right| \frac{\chi_{W}(g)}{\left|H_{g}\right|}=\frac{24}{3} \cdot \frac{-1}{4}=-2 .
$$

If $g=(1234)$, then $C_{G}(g) \cap H=\{(1234)\} \cup\{(1432)\}$ is the decomposition into $H$-conjugacy classes. Hence

$$
\chi_{V}(g)=\frac{24}{6}\left(\frac{\iota}{4}-\frac{\iota}{4}\right)=0
$$

We can easily check that $\left\|\chi_{V}\right\|^{2}=2 \neq 1$, and hence $V$ is a reducible representation of $S_{4}$. Thus, it may happen that although $W$ is irreducible, the induced representation $V$ is reducible. 


\section{Chapter 3}

\section{Conjugacy Classes in $\mathbf{G L}\left(2, \mathbb{F}_{q}\right)$}

In the previous chapter, we have seen that the number of conjugacy classes in a group equals the number of its irreducible representations. In this chapter, we will describe the conjugacy classes in $G=\mathrm{GL}\left(2, \mathbb{F}_{q}\right)$. Its irreducible representations will be described in the next chapter. Recall that

$$
G=\mathrm{GL}\left(2, \mathbb{F}_{q}\right)=\left\{A=\left(\begin{array}{ll}
a & b \\
c & d
\end{array}\right) \mid a, b, c, d \in \mathbb{F}_{q} \text { and } a d-b c \neq 0\right\}
$$

\subsection{The Order of $G$}

Let $A \in G$ denote a typical element. There are $q^{2}-1$ ways to select the pair $(a, b)$, since $a, b$ are elements from $\mathbb{F}_{q}$ but they cannot be both zero at the same time. Given $(a, b)$, there are $q^{2}-q$ ways of selecting the pair $(c, d)$ since $c, d$ are elements in $\mathbb{F}_{q}$ but the vector $\langle c, d\rangle$ cannot be a scalar multiple of $\langle a, b\rangle$. (If it were, we would have $\operatorname{det}(A)=0$.) Therefore,

$$
|G|=\left(q^{2}-1\right)\left(q^{2}-q\right)=q(q+1)(q-1)^{2} .
$$




\subsection{1}

For $a \in G$, let

$$
C(a)=\left\{h^{-1} a h \mid h \in G\right\}
$$

denote its conjugacy class. Recall that the stabilizer subgroup of $a$ is defined to be

$$
G_{a}=\left\{h \in G: h^{-1} a h=a\right\} .
$$

Then we have the basic equation

$$
|C(a)|=\frac{|G|}{\left|G_{a}\right|} .
$$

In other words, the size of the conjugacy class $C(a)$ is equal to the index of the stabilizer $G_{a}$.

The following theorem gives complete information about the conjugacy classes in $G$. Once and for all, we choose an element $\epsilon \in \mathbb{F}_{q}$ such that $t^{2}-\epsilon \in \mathbb{F}_{q}[t]$ is an irreducible polynomial. In other words, $\epsilon$ is not the square of any element in $\mathbb{F}_{q}$.

Theorem 3.1. There are four types of conjugacy classes in $G$.

- Type I classes: Let $x \in \mathbb{F}_{q}$ be a nonzero element, and $a_{x}=\left(\begin{array}{ll}x & 0 \\ 0 & x\end{array}\right)$. Then we have the singleton conjugacy class $C\left(a_{x}\right)=\left\{a_{x}\right\}$. There are $q-1$ such classes.

- Type II classes: Let $x \in \mathbb{F}_{q}$ be a nonzero element, and $b_{x}=\left(\begin{array}{cc}x & 1 \\ 0 & x\end{array}\right)$. Then we have the conjugacy class $C\left(b_{x}\right)$ with $q^{2}-1$ elements. There are $q-1$ such classes.

- Type III classes: Let $x, y \in \mathbb{F}_{q}$ be distinct nonzero elements, and $c_{x, y}=\left(\begin{array}{ll}x & 0 \\ 0 & y\end{array}\right)$. Then we have the conjugacy class $C\left(c_{x, y}\right)$ with $q^{2}+q$ elements. Moreover, $C\left(c_{x, y}\right)=C\left(c_{y, x}\right)$, and hence there are $\left(\begin{array}{c}q-1 \\ 2\end{array}\right)$ such classes corresponding to unordered pairs $\{x, y\}$. 
- Type IV classes: Let $x, y$ be (not necessarily distinct) elements in $\mathbb{F}_{q}$ such that $y \neq 0$. Let $d_{x, y}=\left(\begin{array}{cc}x & \epsilon y \\ y & x\end{array}\right)$. Then we have the conjugacy class $C\left(d_{x, y}\right)$ with $q^{2}-q$ elements. Moreover, $C\left(d_{x, y}\right)=C\left(d_{x,-y}\right)$, and hence there are $\frac{q(q-1)}{2}$ such classes.

The total number of conjugacy classes is

$$
(q-1)+(q-1)+(q-1)(q-2) / 2+q(q-1) / 2=q^{2}-1 .
$$

The theorem will be proved in several steps below.

\subsection{Eigenvalues}

Let $A=\left(\begin{array}{ll}a & b \\ c & d\end{array}\right)$ be an element in $G$. Let $\lambda_{1}, \lambda_{2}$ be the eigenvalues of $A$ in the algebraic closure of $\mathbb{F}_{q}$. There are the following possibilities:

- We have $\lambda_{1}=\lambda_{2}$. Since this common value $=\frac{1}{2} \operatorname{trace}(A)$, it must lie in $\mathbb{F}_{q}$.

(I) If $A$ is diagonalizable, then we say that $A$ is of type $\mathrm{I}$.

(II) If $A$ is not diagonalizable, then we say that $A$ is of type II.

- The other possibility is that $\lambda_{1}, \lambda_{2}$ are distinct. In that case, they are both in $\mathbb{F}_{q}$ or both outside it.

(III) If both $\lambda_{i}$ are in $\mathbb{F}_{q}$, then we say that $A$ is of type III.

(IV) If both $\lambda_{i}$ are outside $\mathbb{F}_{q}$, then we say that $A$ is of type IV.

We can deduce the following information about $A \in G$ from its type. 
- Suppose $\lambda_{1}=\lambda_{2}=x$. If $A$ is of type I, then it is conjugate to the diagonal matrix $a_{x}$. If it is of type II, then it is conjugate to its Jordan canonical form, which is $b_{x}$. Since $x \neq 0$, there are $q-1$ matrices $a_{x}$ or $b_{x}$.

- Suppose $\lambda_{1} \neq \lambda_{2}$. If $A$ is of type III, then it is diagonalizable in $\mathbb{F}_{q}$ and hence conjugate to the matrix $c_{\lambda_{1}, \lambda_{2}}$. Moreover, there are $\left(\begin{array}{c}q-1 \\ 2\end{array}\right)$ such matrices up to interchanging $\lambda_{1}, \lambda_{2}$.

- If $A$ is of type IV, then it will follow from Lemma 3.5 below that it is conjugate to an element of the form $d_{x, y}$.

\subsection{Stabilizers of Group Elements}

Now we will find the stabilizers of elements

$$
a_{x}, \quad b_{x}, \quad c_{x, y}, \quad d_{x, y} .
$$

Proposition 3.2. Let $A=\left(\begin{array}{ll}a & 0 \\ 0 & b\end{array}\right) \in G$, where $a, b \in \mathbb{F}_{q}$. If $a=b$, then the stabilizer of $A$ is $G$. If $a \neq b$, then the stabilizer of $A$ is the set of all diagonal matrices in $G$.

Proof. Let $A=\left(\begin{array}{ll}a & 0 \\ 0 & b\end{array}\right) \in G$. If $a=b$, then $A=a I$. Then for any matrix $P \in G$ we have $P^{-1} A P=P^{-1} a I P=a I$. Hence the stabilizer of $A$ is all of $G$.

Now assume $a \neq b$, and let $P=\left(\begin{array}{cc}c & r \\ s & t\end{array}\right) \in G$. Then

$$
P^{-1} A P=\left(\begin{array}{cc}
\frac{t}{c t-r s} & \frac{-r}{c t-r s} \\
\frac{-s}{c t-r s} & \frac{c}{c t-r s}
\end{array}\right)\left(\begin{array}{ll}
a & 0 \\
0 & b
\end{array}\right)\left(\begin{array}{cc}
c & r \\
s & t
\end{array}\right)=\underbrace{\left(\begin{array}{cc}
\frac{a c t-b r s}{c t-r s} & \frac{a t r-b r t}{c t-r s} \\
\frac{-a s c+b c s}{c t-r s} & \frac{-a s r+b c t}{c t-r s}
\end{array}\right)}_{Q} .
$$


If $P$ is diagonal, then $r=s=0$. In that case $c, t \neq 0$. Then it is easy to see that $Q=A$ and hence $P \in G_{A}$.

Now assume $P \in G_{A}$, and hence $Q=A$. The off-diagonal entries in $Q$ are zero. Since $a \neq b$, this gives equations

- $r=0$ or $t=0$, and

- $s=0$ or $c=0$.

If $r=0$, then $c t \neq 0$ and hence $s=0$. Similarly if $s=0$, then $c t \neq 0$ and hence $r=0$. Thus $r, s$ are both zero or both nonzero. If they are both zero, then $P$ is diagonal. If they are both nonzero, then $c=t=0$. Comparing the $(1,1)$ entry of $Q$ with $A$, we get $\frac{-b r s}{-r s}=a \Longrightarrow a=b$, which is a contradiction. This proves the proposition.

The sizes of the stabilizers and conjugacy classes are easy to determine. If $a=b$, then $\left|G_{A}\right|=|G|$. In that case, the conjugacy class $C(A)$ is a singleton set. If $a \neq b$, then $\left|G_{A}\right|=(q-1)^{2}$. In that case,

$$
|C(A)|=\frac{q(q+1)(q-1)^{2}}{(q-1)^{2}}=q^{2}+q .
$$

Proposition 3.3. Let $A=\left(\begin{array}{ll}d & 1 \\ 0 & d\end{array}\right) \in G$, where $d \in \mathbb{F}_{q}$ and $d \neq 0$. Then the stabilizer of $A$ consists of all upper-triangular nonsingular matrices with equal diagonal entries. In other words,

$$
G_{A}=\left\{\left(\begin{array}{cc}
a_{1} & b_{1} \\
0 & a_{1}
\end{array}\right) \mid a_{1} \neq 0\right\}
$$


Proof. Let $P=\left(\begin{array}{cc}a_{1} & b_{1} \\ b_{2} & a_{2}\end{array}\right) \in G$. Then

$$
\begin{aligned}
P^{-1} A P & =\left(\begin{array}{ll}
\frac{a_{2}}{a_{1} a_{2}-b_{1} b_{2}} & \frac{-b_{1}}{a_{1} a_{2}-b_{1} b_{2}} \\
\frac{-b_{2}}{a_{1} a_{2}-b_{1} b_{2}} & \frac{a_{1}}{a_{1} a_{2}-b_{1} b_{2}}
\end{array}\right)\left(\begin{array}{ll}
d & 1 \\
0 & d
\end{array}\right)\left(\begin{array}{ll}
a_{1} & b_{1} \\
b_{2} & a_{2}
\end{array}\right) \\
& =\underbrace{\left(\begin{array}{cc}
\frac{a_{1} a_{2} d+a_{2} b_{2}-b_{1} b_{2} d}{a_{1} a_{2}-b_{1} b_{2}} & \frac{a_{2}^{2}}{a_{1} a_{2}-b_{1} b_{2}} \\
\frac{-b_{2}^{2}}{a_{1} a_{2}-b_{1} b_{2}} & \frac{-b_{1} b_{2} d-a_{2} b_{2}+a_{1} a_{2} d}{a_{1} a_{2}-b_{1} b_{2}}
\end{array}\right)}_{Q} .
\end{aligned}
$$

If $a_{1}=a_{2}$ and $b_{2}=0$, then it is easy to see that $Q=A$ and hence $P \in G_{A}$.

Conversely assume $P \in G_{A}$, i.e., $Q=A$. Then $b_{2}=0$. Now the $(1,2)$ entry of $Q$ is $=1$, which implies $\frac{a_{2}^{2}}{a_{1} a_{2}}=1 \Longrightarrow a_{1}=a_{2}$. This proves the proposition.

In this case, $\left|G_{A}\right|=q(q-1)$, because $a_{1}, b_{1}$ are arbitrary with $a_{1} \neq 0$. Hence

$$
|C(A)|=\frac{q(q+1)(q-1)^{2}}{q(q-1)}=q^{2}-1
$$

Hence, the total number of elements of types I, II or III is

$$
(q-1)+\left(q^{2}-1\right)(q-1)+\left(q^{2}+q\right)(q-1)(q-2) / 2=\underbrace{\frac{1}{2} q(q+2)(q-1)^{2}}_{(\star)}
$$

Let $F \subseteq G$ be the set of type IV elements. Then

$$
|F|=|G|-(\star)=\frac{1}{2} q^{2}(q-1)^{2} .
$$




\subsection{1}

Recall that we have fixed a non-square element $\epsilon \in \mathbb{F}_{q}$. Identify the field $\mathbb{F}_{q^{2}}$ with $\mathbb{F}_{q}[t] /\left(t^{2}-\epsilon\right)$. When no confusion is likely, we will write $\sqrt{\epsilon}$ for the element $t \in \mathbb{F}_{q^{2}}$. Let

$$
d_{x, y}=\left(\begin{array}{cc}
x & \epsilon y \\
y & x
\end{array}\right)
$$

where $x, y \in \mathbb{F}_{q}$ and $y \neq 0$. Thus there are $q(q-1)$ such matrices.

The characteristic polynomial of $d_{x, y}$ is

$$
p(z)=\operatorname{det}\left(d_{x, y}-z I_{2}\right)=\operatorname{det}\left(\begin{array}{cc}
x-z & \epsilon y \\
y & x-z
\end{array}\right)=z^{2}-2 x z+x^{2}-\epsilon y^{2} .
$$

Its roots are

$$
x \pm y \sqrt{\epsilon} \in \mathbb{F}_{q^{2}} \backslash \mathbb{F}_{q}
$$

and hence $d_{x, y}$ is of type IV. We will find its stabilizer in the next proposition.

Proposition 3.4. (i) Let $A=\left(\begin{array}{ll}x & \epsilon y \\ y & x\end{array}\right)$ be as above. Then the stabilizer $G_{A}$ consists of all matrices of the form $\left(\begin{array}{cc}d & c \epsilon \\ c & d\end{array}\right) \in G$, where $c, d \in \mathbb{F}_{q}$ are arbitrary elements which are not both zero.

(ii) The elements $d_{x, y}$ and $d_{x^{\prime}, y^{\prime}}$ are conjugate, if and only if $x=x^{\prime}$ and $y^{\prime}= \pm y$.

Proof. First we prove (i). Let $P=\left(\begin{array}{ll}a & b \\ c & d\end{array}\right) \in G$. Now $A=x I_{2}+y T$, where $T=\left(\begin{array}{ll}0 & \epsilon \\ 1 & 0\end{array}\right)$ 
and $I_{2}$ is the $2 \times 2$ identity matrix. Hence

$$
P^{-1} A P=x I_{2}+y P^{-1} T P .
$$

Since $y \neq 0$, it follows that $P^{-1} A P=A$ exactly when $P^{-1} T P=T$. Now

$$
P^{-1} T P=\left(\begin{array}{cc}
\frac{d}{a d-b c} & \frac{-b}{a d-b c} \\
\frac{-c}{a d-b c} & \frac{a}{a d-b c}
\end{array}\right)\left(\begin{array}{cc}
0 & \epsilon \\
1 & 0
\end{array}\right)\left(\begin{array}{cc}
a & b \\
c & d
\end{array}\right)=\underbrace{\left(\begin{array}{cc}
\frac{-a b+\epsilon c d}{a d-b c} & \frac{-b^{2}+\epsilon d^{2}}{a d-b c} \\
\frac{a^{2}-\epsilon c^{2}}{a d-b c} & \frac{a b-\epsilon c d}{a d-b c}
\end{array}\right)}_{Q}
$$

First, if $a=d, b=c \epsilon$, then it is easy to see that $Q=T$. Conversely, assume $Q=T$. Then the following three equations hold:

$$
a b=\epsilon c d, \quad-b^{2}+\epsilon d^{2}=a^{2} \epsilon-\epsilon^{2} c^{2}, \quad a^{2}-\epsilon c^{2}=a d-b c .
$$

If $d=0$, then $a b=0$. However, $b \neq 0$, since $\operatorname{det}(P) \neq 0$. Hence we must have $a=0$. Since $c \neq 0$, we must have $b=\epsilon c$. Assume $d \neq 0$. Then we have $a d \epsilon-b c \epsilon=-b^{2}+\epsilon d^{2}$. After substituting $c=a b / \epsilon d$ and rearranging terms, we get

$$
a\left(\epsilon d^{2}-b^{2}\right)=d\left(\epsilon d^{2}-b^{2}\right)
$$

But $\epsilon d^{2} \neq b^{2}$, since $\epsilon$ is a non-square element of $\mathbb{F}_{q}$. Hence we must have $a=d$, and then $b=\epsilon c$. Thus, in either case $P$ must have the form given above. This proves (i). 
Now we prove (ii). Let $P=\left(\begin{array}{cc}a & -\epsilon c \\ c & -a\end{array}\right) \in G$. Then

$$
\begin{aligned}
P^{-1} d_{x, y} P & =\left(\begin{array}{cc}
\frac{-a}{\epsilon c^{2}-a^{2}} & \frac{\epsilon c}{\epsilon c^{2}-a^{2}} \\
\frac{-c}{\epsilon c^{2}-a^{2}} & \frac{a}{\epsilon c^{2}-a^{2}}
\end{array}\right)\left(\begin{array}{ll}
x & \epsilon y \\
y & x
\end{array}\right)\left(\begin{array}{cc}
a & -\epsilon c \\
c & -a
\end{array}\right)=\left(\begin{array}{cc}
\frac{-a x+\epsilon c y}{\epsilon c^{2}-a^{2}} & \frac{\epsilon c x-\epsilon a y}{\epsilon c^{2}-a^{2}} \\
\frac{-c x+a y}{\epsilon c^{2}-a^{2}} & \frac{a x-\epsilon c y}{\epsilon c^{2}-a^{2}}
\end{array}\right)\left(\begin{array}{ll}
a & -\epsilon c \\
c & -a
\end{array}\right) \\
& =\left(\begin{array}{cc}
x & -\epsilon y \\
-y & x
\end{array}\right)=d_{x,-y} .
\end{aligned}
$$

Hence $d_{x, y}, d_{x,-y}$ are conjugate. Conversely, suppose that $d_{x, y}$ and $d_{x^{\prime}, y^{\prime}}$ are conjugate. Then they must have the same trace and determinant. Hence $2 x=2 x^{\prime} \Longrightarrow x=x^{\prime}$. Moreover, $x^{2}-\epsilon y^{2}=x^{2}-\epsilon y^{\prime 2} \Longrightarrow y^{\prime}= \pm y$. The proposition is proved.

Since the stabilizer of $d_{x, y}$ has size $q^{2}-1$, it follows that $C\left(d_{x, y}\right)=\frac{q(q+1)(q-1)^{2}}{q^{2}-1}=q^{2}-q$. Moreover, there are $q(q-1) / 2$ such conjugacy classes. Let $T \subseteq G$ be the subset of elements which are conjugate to some $d_{x, y}$. Then

$$
|T|=\left(q^{2}-q\right) \frac{q(q-1)}{2}=\frac{q^{2}(q-1)^{2}}{2} .
$$

Lemma 3.5. Each type IV element is conjugate to $d_{x, y}$ for some $x, y$.

Proof. We have $T \subseteq F$, since each $d_{x, y}$ is of type IV. However, $|T|=|F|$ and hence these two sets are equal. This proves the lemma.

At this point, Theorem 3.1 is completely proved. The following table summarizes the information about these four different types of conjugacy classes. 


\begin{tabular}{|c||r|r|}
\hline Class representative & Class size & Number of Classes \\
\hline \hline$a_{x}$ & 1 & $q-1$ \\
\hline$b_{x}$ & $q^{2}-1$ & $q-1$ \\
\hline$c_{x, y}$ & $q^{2}+q$ & $(q-1)(q-2) / 2$ \\
\hline$d_{x, y}$ & $q^{2}-q$ & $q(q-1) / 2$ \\
\hline
\end{tabular}

The total number of conjugacy classes is

$$
(q-1)+(q-1)+\frac{(q-1)(q-2)}{2}+\frac{q(q-1)}{2}=q^{2}-1 .
$$

Hence $\operatorname{GL}\left(2, \mathbb{F}_{q}\right)$ has the same number of mutually non-isomorphic irreducible representations. We will construct them explicitly in the next chapter. 


\section{Chapter 4}

\section{The Irreducible Representations of}

\section{$\mathbf{G L}\left(2, \mathbb{F}_{q}\right)$}

In the previous chapter we have discussed the conjugacy classes in $G=\mathrm{GL}\left(2, \mathbb{F}_{q}\right)$. It turns out that there are altogether $q^{2}-1$ classes. According to Theorem 2.16, we have the same number of irreducible representations of $G$ up to isomorphism. In this chapter, we will construct these irreducible representations.

In summary, it will turn out that there are four kinds of irreducible representations:

- For every group homomorphism $\alpha: \mathbb{F}_{q}^{*} \longrightarrow \mathbb{C}^{*}$, we have a one-dimensional representation $U_{\alpha}$ and a $q$-dimensional representation $V_{\alpha}$.

- For a pair of such homomorphisms $\alpha, \beta$ (such that $\alpha \neq \beta$ ) we have a $(q+1)$-dimensional representation $W_{\alpha, \beta}$. These are usually called principal series representations.

- Let $E \supseteq \mathbb{F}_{q}$ be a quadratic extension of $\mathbb{F}_{q}$. For every group homomorphism $\phi: E^{*} \longrightarrow \mathbb{C}^{*}$ such that $\phi \neq \phi^{q}$, we have a $(q-1)$-dimensional representation $X_{\phi}$. These are usually called discrete series representations. 


\subsection{The Permutation Representation}

\subsubsection{Projective Line Over a Field}

Definition 4.1. (Homogeneous Coordinates)

Let $F$ be a field. Consider the column matrix $\left(\begin{array}{l}x \\ y\end{array}\right)$, where $x, y \in F$ and $x, y$ are not both zero. We write $\left(\begin{array}{l}x \\ y\end{array}\right) \equiv\left(\begin{array}{l}\lambda x \\ \lambda y\end{array}\right)$, for any $\lambda \in F^{*}$. This is an equivalence relation on column vectors. The projective line over $F$ is the set of all such equivalence classes. It is denoted by $\mathbb{P}^{1}(F)$.

Note that the equivalence classes in $\mathbb{P}^{1}\left(\mathbb{F}_{q}\right)$ are represented by points $\left(\begin{array}{l}1 \\ x\end{array}\right)$ for $x \in \mathbb{F}_{q}$, together with $\left(\begin{array}{l}0 \\ 1\end{array}\right)$. The point $\left(\begin{array}{l}0 \\ 1\end{array}\right)$ is usually called the point at infinity. Hence we can say that $\mathbb{P}^{1}\left(\mathbb{F}_{q}\right)$ has $q+1$ distinct points.

\subsubsection{Permutation Representation}

Let

$$
r_{1}=\left(\begin{array}{l}
1 \\
0
\end{array}\right), r_{2}=\left(\begin{array}{l}
1 \\
1
\end{array}\right), \ldots, r_{q}=\left(\begin{array}{c}
1 \\
q-1
\end{array}\right), r_{\infty}=\left(\begin{array}{l}
0 \\
1
\end{array}\right) .
$$

Let $\left[r_{i}\right]$ denote the equivalence class of $r_{i}$. Let $M$ be a vector space over $\mathbb{F}_{q}$ with basis

$$
\left\{\left[r_{1}\right],\left[r_{2}\right], \ldots,\left[r_{q}\right],\left[r_{\infty}\right]\right\}
$$

Each element $v \in M$ has a unique representation $v=\sum c_{i}\left[r_{i}\right]$. Let $g \in G$ be a $2 \times 2$ matrix, and consider the matrix multiplication $g . r_{i}$. Then we have $\left[g . r_{i}\right]=\left[r_{j}\right]$ for some $j$. 
Define $\rho_{g}: M \longrightarrow M$ by the formula

$$
\rho_{g}(v)=\sum c_{i}\left[g \cdot r_{i}\right] .
$$

It is easy to see that this converts $M$ into a representation of $G$. This is called the permutation representation of $G$ on $\mathbb{P}^{1}\left(\mathbb{F}_{q}\right)$. Now we will calculate the character values of this representation. Then we will check whether $M$ is an irreducible representation.

\subsubsection{Character Values of the Permutation Representation}

Recall the four types of conjugacy classes of $G$ that we have discussed in the previous chapter.

$$
c_{x, y}=\left(\begin{array}{ll}
x & 0 \\
0 & y
\end{array}\right), \quad a_{x}=\left(\begin{array}{ll}
x & 0 \\
0 & x
\end{array}\right), \quad b_{x}=\left(\begin{array}{ll}
x & 1 \\
0 & x
\end{array}\right), \quad d_{x, y}=\left(\begin{array}{cc}
x & \epsilon y \\
y & x
\end{array}\right) .
$$

For any of these elements $g \in G$, we will see how $\rho_{g}$ acts on $M$. The character of the permutation representation is computed by the formula

$$
\chi_{M}(g)=\text { number of points fixed by } \rho_{g} .
$$

\subsection{Character Values of Permutation Representation}

Let $r \in \mathbb{F}_{q}$. Then $\rho_{c_{x, y}}\left(\left(\begin{array}{l}1 \\ r\end{array}\right)\right)=\left(\begin{array}{ll}x & 0 \\ 0 & y\end{array}\right)\left(\begin{array}{l}1 \\ r\end{array}\right)=\left(\begin{array}{l}x \\ y r\end{array}\right)$. In order to calculate the character value, we will see when the element $\left(\begin{array}{l}1 \\ r\end{array}\right)$ is fixed by $\rho_{c_{x, y}}$, that is, $\left(\begin{array}{c}x \\ y r\end{array}\right)=\lambda\left(\begin{array}{l}1 \\ r\end{array}\right)$. This implies, $x=\lambda$ and $y r=\lambda r \Longrightarrow y r=x r \Longrightarrow(x-y) r=0$, since $x \neq y \Longrightarrow r=0$. Also 
$\left.\rho_{c_{x, y}}\left(\begin{array}{l}0 \\ 1\end{array}\right)\right)=\left(\begin{array}{ll}x & 0 \\ 0 & y\end{array}\right)\left(\begin{array}{l}0 \\ 1\end{array}\right)=\left(\begin{array}{l}0 \\ y\end{array}\right)=y\left(\begin{array}{l}0 \\ 1\end{array}\right)=\left(\begin{array}{l}0 \\ 1\end{array}\right)$.

Therefore, the elements $\left(\begin{array}{l}1 \\ 0\end{array}\right),\left(\begin{array}{l}0 \\ 1\end{array}\right)$ of $M$ are fixed by $\rho_{c_{x, y}}$. Hence, $\chi_{M}\left(c_{x, y}\right)=2$.

Now $\rho_{a_{x}}\left(\left(\begin{array}{l}1 \\ r\end{array}\right)\right)=\left(\begin{array}{ll}x & 0 \\ 0 & x\end{array}\right)\left(\begin{array}{l}1 \\ r\end{array}\right)=\left(\begin{array}{l}x \\ x r\end{array}\right)=x\left(\begin{array}{l}1 \\ r\end{array}\right)=\left(\begin{array}{l}1 \\ r\end{array}\right)$.

Also, $\left.\rho_{a_{x}}\left(\begin{array}{l}0 \\ 1\end{array}\right)\right)=\left(\begin{array}{ll}x & 0 \\ 0 & x\end{array}\right)\left(\begin{array}{l}0 \\ 1\end{array}\right)=\left(\begin{array}{l}0 \\ x\end{array}\right)=x\left(\begin{array}{l}0 \\ 1\end{array}\right)=\left(\begin{array}{l}0 \\ 1\end{array}\right)$.

Therefore, all the elements of $M$ are fixed by $\rho_{a_{x}}$. Hence, $\chi_{M}\left(a_{x}\right)=q+1$. Now $\left.\rho_{b_{x}}\left(\begin{array}{l}1 \\ r\end{array}\right)\right)=$ $\left(\begin{array}{ll}x & 1 \\ 0 & x\end{array}\right)\left(\begin{array}{l}1 \\ r\end{array}\right)=\left(\begin{array}{c}x+r \\ x r\end{array}\right)$

In order to calculate the character value, we will see when the element $\left(\begin{array}{l}1 \\ r\end{array}\right)$ is fixed by $\rho_{b_{x}}$, that is, $\left(\begin{array}{c}x+r \\ x r\end{array}\right)=\lambda\left(\begin{array}{l}1 \\ r\end{array}\right)$. This implies that, $(x+r)=\lambda$ and $x r=\lambda r \Longrightarrow x r=(x+r) r \Longrightarrow$ $x r=x r+r^{2} \Longrightarrow r^{2}=0 \Longrightarrow r=0$.

Also, $\left.\rho_{b_{x}}\left(\begin{array}{l}0 \\ 1\end{array}\right)\right)=\left(\begin{array}{ll}x & 1 \\ 0 & x\end{array}\right)\left(\begin{array}{l}0 \\ 1\end{array}\right)=\left(\begin{array}{l}1 \\ x\end{array}\right) \neq\left(\begin{array}{l}0 \\ 1\end{array}\right)$.

Therefore, only one element of $M$ is fixed by $\rho_{b_{x}}$. Hence, $\chi_{M}\left(b_{x}\right)=1$.

Now $\rho_{d_{x, y}}\left(\begin{array}{l}1 \\ r\end{array}\right)=\left(\begin{array}{ll}x & \epsilon y \\ y & x\end{array}\right)\left(\begin{array}{l}1 \\ r\end{array}\right)=\left(\begin{array}{c}x+\epsilon y r \\ x r+y\end{array}\right)$.

In order to calculate the character value, we will see when the element $\left(\begin{array}{l}1 \\ r\end{array}\right)$ is fixed by $\rho_{d_{x, y}}$, 
that is, $\left(\begin{array}{c}x+\epsilon y r \\ x r+y\end{array}\right)=\lambda\left(\begin{array}{l}1 \\ r\end{array}\right)$. This implies, $x+\epsilon y r=\lambda$ and $x r+y=\lambda r \quad \Longrightarrow \quad x r+$ $y=(x+\epsilon y r) r \Longrightarrow x r+y=x r+\epsilon y r^{2} \Longrightarrow y=\epsilon y r^{2} \Longrightarrow y\left(1-\epsilon r^{2}\right)=0$ since $y \neq 0 \Longrightarrow 1-\epsilon r^{2}=0 \Longrightarrow r=\sqrt{\frac{1}{\epsilon}} \notin \mathbb{F}_{q}$.

Also, $\left.\rho_{d_{x, y}}\left(\begin{array}{l}0 \\ 1\end{array}\right)\right)=\left(\begin{array}{cc}x & \epsilon y \\ y & x\end{array}\right)\left(\begin{array}{l}0 \\ 1\end{array}\right)=\left(\begin{array}{l}\epsilon y \\ x\end{array}\right)$

In order to calculate the character value, we will see when the element $\left(\begin{array}{l}0 \\ 1\end{array}\right)$ is fixed by $\rho_{d_{x, y}}$ $\left(\begin{array}{c}\epsilon y \\ x\end{array}\right)=\lambda\left(\begin{array}{l}0 \\ 1\end{array}\right) \Longrightarrow \epsilon y=0$, which is not possible because $y, \epsilon \neq 0$. Therefore, no element of $M$ is fixed by $\rho_{d_{x, y}}$. Hence, $\chi_{M}\left(d_{x, y}\right)=0$.

The following table gives the character values of $M$ at different conjugacy classes.

\begin{tabular}{|c||c|c|c|c|}
\hline & $a_{x}$ & $b_{x}$ & $c_{x, y}$ & $d_{x, y}$ \\
\hline Number of elements & & & & \\
in the conjugacy class & $q-1$ & $(q-1)^{2}(q+1)$ & $\frac{q(q-1)(q-2)(q+1)}{2}$ & $\frac{q^{2}(q-1)^{2}}{2}$ \\
\hline \hline$\chi_{M}$ & $q+1$ & 1 & 2 & 0 \\
\hline
\end{tabular}

Note that the character values are in fact independent of $x, y$. To check irreducibility of $M$, we use 
Theorem 2.22(i). We have

$$
\begin{aligned}
\left\|\chi_{M}\right\|^{2} & =\left\langle\chi_{M}, \chi_{M}\right\rangle \\
& =\frac{1}{|G|}\left[(q-1)^{2}(q+1)+(q-1)(q+1)^{2}+\left(\frac{q(q-1)(q-2)(q+1)}{2}\right) 2.2\right] \\
& =\frac{1}{q(q-1)^{2}(q+1)}\left[(q-1)^{2}(q+1)+(q-1)(q+1)^{2}+2 q(q-1)(q-2)(q+1)\right] \\
& =\frac{1}{q(q-1)^{2}(q+1)}(q-1)(q+1)\left[(q-1)+(q+1)+\left(2 q^{2}-4 q\right)\right] \\
& =\frac{1}{q(q-1)}\left[\left(2 q^{2}-2 q\right)\right]=2 \neq 1 .
\end{aligned}
$$

Hence $M$ is not irreducible.

\subsection{Complementary $q$-Dimensional Representation}

We will denote the trivial representation by $\mathbb{T}$. First we will find how many copies of trivial representation are in the permutation representation. By Proposition 2.23 ,

$$
\begin{aligned}
& \left\langle\chi_{M}, \chi_{\mathbb{T}}\right\rangle \\
= & \frac{1}{|G|}\left[(q-1)^{2}(q+1) 1.1+(q-1)(q+1) .1+\left(\frac{q(q-1)(q-2)(q+1)}{2}\right) 2.1+\left(\frac{q^{2}(q-1)^{2}}{2}\right) 0.1\right] \\
= & \frac{1}{q(q-1)^{2}(q+1)}\left[(q-1)^{2}(q+1)+(q-1)(q+1)+q(q-1)(q-2)(q+1)\right] \\
= & \frac{1}{q(q-1)^{2}(q+1)}(q-1)(q+1)\left[(q-1)+1+\left(q^{2}-2 q\right)\right] \\
= & \frac{1}{q(q-1)}\left(q^{2}-q\right)=1 .
\end{aligned}
$$

Therefore, there is only one copy of trivial representation in the permutation representation. Explicitly, we can say that

$$
\mathbb{T}=\operatorname{span}\left\{\left[r_{1}\right]+\left[r_{2}\right]+\cdots+\left[r_{q}\right]+\left[r_{\infty}\right]\right\}
$$


Therefore, $M=\mathbb{T} \bigoplus V$, where $V$ is the complementary $q$-dimensional representation. We already know the character values of $M$ and $\mathbb{T}$, hence we can compute the character values of $V$ using Proposition 2.21(iv). They are as follows:

\begin{tabular}{|c||c|c|c|c|}
\hline & $a_{x}$ & $b_{x}$ & $c_{x, y}$ & $d_{x, y}$ \\
\hline \hline$\chi_{V}$ & $q$ & 0 & 1 & -1 \\
\hline
\end{tabular}

Then

$$
\begin{aligned}
& \left\|\chi_{V}\right\|^{2} \\
& =\frac{1}{|G|}\left[(q-1)^{2}(q+1) .0+(q-1) q^{2}+\left(\frac{q(q-1)(q-2)(q+1)}{2}\right) 1.1+\left(\frac{q^{2}(q-1)^{2}}{2}\right)(-1)(-1)\right] \\
& =\frac{1}{q(q-1)^{2}(q+1)}\left[(q-1) q^{2}+\left(\frac{q(q-1)(q-2)(q+1)}{2}\right)+\left(\frac{q^{2}(q-1)^{2}}{2}\right)\right] \\
& =\frac{1}{q(q-1)^{2}(q+1)} q(q-1)\left[\frac{2 q+q^{2}-q-2+q^{2}-q}{2}\right] \\
& =\frac{1}{\left(q^{2}-1\right)}\left[\left(q^{2}-1\right)\right]=1
\end{aligned}
$$

Hence $V$ is an irreducible representation.

\subsection{One-dimensional Representations}

In this section we will define some one-dimensional representations of $G=\mathrm{GL}\left(2, \mathbb{F}_{q}\right)$. It will turn out later that these are all of its one-dimensional representations up to isomorphism.

Define a map

$$
\delta: G \rightarrow \mathbb{F}_{q}^{*}, \quad \delta(g)=\operatorname{det}(g)
$$

Since

$$
\operatorname{det}\left(g_{1} g_{2}\right)=\operatorname{det}\left(g_{1}\right) \operatorname{det}\left(g_{2}\right), \quad \text { and } \quad \operatorname{det}\left(g^{-1}\right)=\operatorname{det}(g)^{-1}
$$


it follows that $\delta$ is a group homomorphism.

If $F$ is a field, then a homomorphism of multiplicative groups $F^{*} \longrightarrow \mathbb{C}^{*}$ is called a character of $F^{*}$. In our context, we will usually have $F=\mathbb{F}_{q}$ or $\mathbb{F}_{q^{2}}$.

\subsubsection{Characters of $\mathbb{F}_{q}^{*}$}

We will find all the group homomorphisms

$$
\alpha: \mathbb{F}_{q}^{*} \rightarrow \mathbb{C}^{*}
$$

Recall that $\mathbb{F}_{q}^{*}$ is a cyclic group of order $q-1$ (see [3, Section 14.3]). Let $r$ be one of its generators. Then $1=\alpha(1)=\alpha\left(r^{q-1}\right)=\alpha(r)^{q-1}$, and hence $\alpha(r)$ must be a $(q-1)$-th root of unity. Let

$$
z=\exp \left(\frac{2 k \pi \iota}{q-1}\right)
$$

be a $(q-1)$-th root of unity for some $k$ in the range $0 \leqslant k \leqslant q-2$. Then the formula $\alpha\left(r^{i}\right)=z^{i}$ defines a group homomorphism $\mathbb{F}_{q}^{*} \stackrel{\alpha}{\longrightarrow} \mathbb{C}^{*}$, and all possible $\alpha$ are obtained this way.

Since $\alpha(x)$ is a root of unity for any $x \in \mathbb{F}_{q}^{*}$, we have $\alpha^{-1}(x)=\overline{\alpha(x)}$.

\subsection{2}

Now consider the composite map

$$
G \stackrel{\delta}{\longrightarrow} \mathbb{F}_{q}^{*} \stackrel{\alpha}{\longrightarrow} \mathbb{C}^{*}
$$

It defines a one-dimensional (and hence irreducible) representation of $G$. Let $U_{\alpha} \simeq \mathbb{C}$ denote the underlying vector space. Thus we have defined $q-1$ such representations for all possible values of $k$.

Since the composite map sends $\left(\begin{array}{cc}x_{1} & x_{2} \\ x_{3} & x_{4}\end{array}\right) \in G$ to $\alpha\left(x_{1} x_{4}-x_{2} x_{3}\right)$, the character table of $U_{\alpha}$ is as 
follows:

\begin{tabular}{|c||c|c|c|c|}
\hline & $a_{x}$ & $b_{x}$ & $c_{x, y}$ & $d_{x, y}$ \\
\hline \hline$\chi_{U_{\alpha}}$ & $\alpha\left(x^{2}\right)$ & $\alpha\left(x^{2}\right)$ & $\alpha(x y)$ & $\alpha\left(x^{2}-\epsilon y^{2}\right)$ \\
\hline
\end{tabular}

\subsection{3}

Now, we will define another representation of $G$ using $V$ and $U_{\alpha}$. Let

$$
V_{\alpha}=V \otimes U_{\alpha}
$$

Since $\chi_{V_{\alpha}}=\chi_{V} \chi_{U_{\alpha}}$, the character table for $V_{\alpha}$ is as follows:

\begin{tabular}{|c||c|c|c|c|}
\hline & $a_{x}$ & $b_{x}$ & $c_{x, y}$ & $d_{x, y}$ \\
\hline \hline$V_{\alpha}$ & $q \alpha(x)^{2}$ & 0 & $\alpha(x) \alpha(y)$ & $-\alpha\left(x^{2}-\epsilon y^{2}\right)$ \\
\hline
\end{tabular}

If we take $k=0$, then $z=1$. In that case $U_{\alpha}$ is the trivial representation and $V_{\alpha} \simeq V$. For $q-1$ possibilities of $\alpha$, we get $(q-1)$ possibilities for $V_{\alpha}$. Henceforth, we denote the one-dimension trivial representation by $U_{\mathbb{T}}$.

Lemma 4.2. The representation $V_{\alpha}$ is irreducible.

PROOF. A non-trivial decomposition $V_{\alpha} \cong E \oplus E^{\prime}$ would correspond to a non-trivial decomposition

$$
V \cong V_{\alpha} \otimes U_{\alpha^{-1}} \cong\left(E \otimes U_{\alpha^{-1}}\right) \oplus\left(E^{\prime} \otimes U_{\alpha^{-1}}\right)
$$

But this is impossible, since $V$ is known to be irreducible. 


\subsection{The Representations $W_{\alpha, \beta}$}

Consider the Borel subgroup

$$
B=\left\{\left(\begin{array}{ll}
a & b \\
0 & d
\end{array}\right): a, d \in \mathbb{F}_{q}^{*} \text { and } b \in \mathbb{F}_{q}\right\}
$$

in $G$. Its order is $(q-1)^{2} q$, and hence $[G: B]=q+1$.

Also, consider the group of upper triangular unipotent matrices $N=\left\{\left(\begin{array}{ll}1 & b \\ 0 & 1\end{array}\right): b \in \mathbb{F}_{q}\right\}$.

Clearly, $N \subset B \subset G$.

Lemma 4.3. With notation as above, $N$ is a normal subgroup of $B$.

Proof. Let $\left(\begin{array}{ll}a & b \\ 0 & d\end{array}\right) \in B$ and let $\left(\begin{array}{ll}1 & c \\ 0 & 1\end{array}\right) \in N$. Consider

$$
\left(\begin{array}{ll}
a & b \\
0 & d
\end{array}\right)\left(\begin{array}{ll}
1 & c \\
0 & 1
\end{array}\right)\left(\begin{array}{ll}
a & b \\
0 & d
\end{array}\right)^{-1}=\left(\begin{array}{cc}
a & a c+b \\
0 & d
\end{array}\right)\left(\begin{array}{cc}
a^{-1} & -b(a d)^{-1} \\
0 & d^{-1}
\end{array}\right)=\left(\begin{array}{cc}
1 & a c d^{-1} \\
0 & 1
\end{array}\right),
$$

which is in $N$. Hence $N$ is normal in $B$.

Let $B / N$ denote the quotient group consisting of left-cosets of $N$ in $B$. Define $f: B \rightarrow \mathbb{F}_{q}^{*} \times \mathbb{F}_{q}^{*}$ by the formula $f\left(\left(\begin{array}{ll}a & b \\ 0 & d\end{array}\right)\right)=(a, d)$.

Lemma 4.4. The morphism $f$ is surjective with kernel $N$. Hence we have an isomorphism $B / N \simeq$ $\mathbb{F}_{q}^{*} \times \mathbb{F}_{q}^{*}$

PROOF. It is immediate that $f$ is surjective. Moreover, $g=\left(\begin{array}{ll}a & b \\ 0 & d\end{array}\right)$ is in the kernel of $f$, if and only if $(a, d)=(1,1)$ which happens exactly when $g \in N$. This completes the proof. 


\subsection{1}

Let $\alpha, \beta: \mathbb{F}_{q}^{*} \longrightarrow \mathbb{C}^{*}$ be two homomorphisms. This gives a homomorphism $B \longrightarrow \mathbb{C}^{*}$ which takes $\left(\begin{array}{ll}a & b \\ 0 & d\end{array}\right)$ to $\alpha(a) \beta(d)$. Thus we get a one-dimensional representation of $B$. Let $W_{\alpha, \beta}$ be the representation induced from $B$ to $G$. We know that

$$
\operatorname{dim} W_{\alpha, \beta}=[G: B]=q+1 .
$$

We will find the character of $W_{\alpha, \beta}$ using Proposition 2.30. The statements below about conjugacy classes and stabilizers follow from Propositions 3.2 and 3.3 in Chapter 3 . We have

$$
\begin{array}{r}
C_{G}\left(c_{x, y}\right) \bigcap B=C_{B}\left(c_{x, y}\right) \bigcup C_{B}\left(c_{y, x}\right), \\
\text { where } c_{x, y}=\left(\begin{array}{ll}
x & 0 \\
0 & y
\end{array}\right) \text { and } c_{y, x}=\left(\begin{array}{ll}
y & 0 \\
0 & x
\end{array}\right) \text {. Moreover, } \\
\left|G_{c_{x, y}}\right|=\left|B_{c_{x, y}}\right|=\left|B_{c_{y, x}}\right|=(q-1)^{2} .
\end{array}
$$

Hence

$$
\begin{aligned}
\chi_{W_{\alpha, \beta}}\left(c_{x, y}\right) & =\chi_{\operatorname{Ind}_{B}^{G}}\left(c_{x, y}\right)=\left|G_{c_{x, y}}\right|\left(\frac{\alpha(x) \beta(y)}{\left|B_{c_{x, y}}\right|}+\frac{\alpha(y) \beta(x)}{\left|B_{c_{y, x}}\right|}\right) \\
& =(q-1)^{2}\left(\frac{\alpha(x) \beta(y)}{(q-1)^{2}}+\frac{\alpha(y) \beta(x)}{(q-1)^{2}}\right)=\alpha(x) \beta(y)+\alpha(y) \beta(x) .
\end{aligned}
$$

We have $C_{G}\left(a_{x}\right) \cap B=C_{B}\left(a_{x}\right)$, and

$$
\left|G_{a_{x}}\right|=|G|=q(q-1)^{2}(q+1), \quad\left|B_{a_{x}}\right|=|B|=q(q-1)^{2} .
$$


Thus

$$
\begin{aligned}
\chi_{W_{\alpha, \beta}}\left(a_{x}\right) & =\chi_{\operatorname{Ind}_{B}^{G}}\left(a_{x}\right)=\left|G_{b_{x}}\right|\left(\frac{\alpha(x) \beta(x)}{\left|B_{a_{x}}\right|}\right) \\
& =q(q-1)^{2}(q+1)\left(\frac{\alpha(x) \beta(x)}{q(q-1)^{2}}\right)=(q+1) \alpha(x) \beta(x) .
\end{aligned}
$$

Also, $C_{G}\left(b_{x}\right) \bigcap B=C_{B}\left(b_{x}\right)$ and $\left|G_{b_{x}}\right|=\left|B_{b_{x}}\right|=q(q-1)$. Thus

$$
\begin{aligned}
\chi_{W_{\alpha, \beta}}\left(b_{x}\right) & =\chi_{\operatorname{Ind}_{B}^{G}}\left(b_{x}\right)=\left|G_{b_{x}}\right|\left(\frac{\alpha(x) \beta(x)}{\left|B_{b_{x}}\right|}\right) \\
& =q(q-1)\left(\frac{\alpha(x) \beta(x)}{q(q-1)}\right)=\alpha(x) \beta(x) .
\end{aligned}
$$

Lastly, $C\left(d_{x, y}\right) \bigcap B=\emptyset$, and thus

$$
\chi_{W_{\alpha, \beta}}\left(d_{x, y}\right)=\chi_{\operatorname{Ind}_{B}^{G}}\left(d_{x, y}\right)=0 .
$$

In conclusion, the character table of $W_{\alpha, \beta}$ is as follows:

\begin{tabular}{|c||c|c|c|c|}
\hline & $a_{x}$ & $b_{x}$ & $c_{x, y}$ & $d_{x, y}$ \\
\hline \hline$\chi_{W_{\alpha, \beta}}$ & $(q+1) \alpha(x) \beta(x)$ & $\alpha(x) \beta(x)$ & $\alpha(x) \beta(y)+\alpha(y) \beta(x)$ & 0 \\
\hline
\end{tabular}

From the table, we observe that $\chi_{W_{\alpha, \beta}}=\chi_{W_{\beta, \alpha}}$. We know that two representations are isomorphic if they have the same character values, hence $W_{\alpha, \beta} \cong W_{\beta, \alpha}$. Also, by comparing the character values of $U_{\alpha}, V_{\alpha}$ and $W_{\alpha, \alpha}$, we observe that $W_{\alpha, \alpha} \cong U_{\alpha} \oplus V_{\alpha}$.

Now, we will prove that $W_{\alpha, \beta}$ is irreducible for $\alpha \neq \beta$ using the following lemma. Choose a generator $r$ for the multiplicative group $\mathbb{F}_{q}^{*}$. Let $\alpha: \mathbb{F}_{q}^{*} \rightarrow \mathbb{C}^{*}$ be a character. Then $\alpha(r)=z$, where $z$ is a complex $(q-1)$-th root of unity. 
Lemma 4.5. With notation as above,

$$
\sum_{x \in \mathbb{F}_{q}^{*}} \alpha(x)= \begin{cases}q-1 & \text { if } \alpha \text { is trivial } \\ 0 & \text { if } \alpha \text { is non-trivial. }\end{cases}
$$

Proof. For $\alpha$ is trivial, $\alpha(x)=1$ for all $x \in \mathbb{F}_{q}^{*}$, hence the sum is $q-1$. If $\alpha$ is non-trivial, then the sum is $1+z+z^{2}+\cdots+z^{q-2}=\frac{z^{q-1}-1}{z-1}=0$.

The same argument can be used to prove the following result. It will prove useful in Section 4.6

Lemma 4.6. If $\beta: \mathbb{F}_{q^{2}}^{*} \longrightarrow \mathbb{C}^{*}$ is a character, then

$$
\sum_{w \in \mathbb{F}_{q^{2}}^{*}} \beta(w)= \begin{cases}q^{2}-1 & \text { if } \beta \text { is trivial } \\ 0 & \text { if } \beta \text { is non-trivial. }\end{cases}
$$

\subsection{2}

Now let $\alpha, \beta: \mathbb{F}_{q}^{*} \longrightarrow \mathbb{C}^{*}$ be two distinct characters. Then

$$
\mathbb{F}_{q}^{*} \rightarrow \mathbb{C}^{*}, \quad x \rightarrow \alpha(x) \overline{\beta(x)},
$$


is a non-trivial character to which Lemma 4.5 applies. Hence

$$
\begin{aligned}
& \left\|\chi_{W_{\alpha, \beta}}\right\|^{2} \\
& =\frac{1}{|G|}\left[\left(\frac{q^{2}+q}{2}\right) \sum_{x, y \in \mathbb{F}_{q}^{*}, x \neq y}(\alpha(x) \beta(y)+\alpha(y) \beta(x) \overline{(\alpha(x) \beta(y)+\alpha(y) \beta(x))}\right. \\
& \left.+\left(q^{2}-1\right) \sum_{x \in \mathbb{F}_{q}^{*}} \alpha(x) \overline{\alpha(x)}+\sum_{x \in \mathbb{F}_{q}^{*}}(q+1)^{2} \alpha(x) \overline{\alpha(x)}\right] \\
& =\frac{1}{q(q-1)^{2}(q+1)}\left[\frac { q ^ { 2 } + q } { 2 } \left(\sum_{x, y \in \mathbb{F}_{q}^{*}, x \neq y}[\alpha(x) \beta(y) \overline{\alpha(x) \beta(y)}+\alpha(x) \beta(y) \overline{\alpha(y) \beta(x)}\right.\right. \\
& \left.+\alpha(y) \beta(x) \overline{\alpha(x) \beta(y)}+\alpha(y) \beta(x) \overline{\alpha(y) \beta(x)}])+\left(q^{2}-1\right) \sum_{x \in \mathbb{F}_{q}^{*}} 1+(q+1)^{2} \sum_{x \in \mathbb{F}_{q}^{*}} 1\right] \\
& =\frac{1}{q(q-1)^{2}(q+1)}\left[\frac{q^{2}+q}{2}\left(\sum_{x, y \in \mathbb{F}_{q}^{*}, x \neq y}[1+\alpha(x) \overline{\alpha(y)} \overline{\beta(x)} \beta(y)+\overline{\alpha(x)} \alpha(y) \beta(x) \overline{\beta(y)}+1]\right)\right. \\
& \left.+\left(q^{2}-1\right)(q-1)+(q+1)^{2}(q-1)\right] \\
& =\frac{1}{q(q-1)^{2}(q+1)}\left[\frac { q ^ { 2 } + q } { 2 } \left(\sum_{x, y \in \mathbb{F}_{q}^{*}, x \neq y} 2+\sum_{x, y \in \mathbb{F}_{q}^{*}, x \neq y} \alpha(x) \overline{\alpha(y)} \overline{\beta(x)} \beta(y)\right.\right. \\
& \left.+\sum_{x \in \mathbb{F}_{q}^{*}} \alpha(x) \overline{\alpha(x)} \overline{\beta(x)} \beta(x)+\sum_{x, y \in \mathbb{F}_{q}^{*}, x \neq y} \overline{\alpha(x)} \alpha(y) \beta(x) \overline{\beta(y)}+\sum_{x \in \mathbb{F}_{q}^{*}} \overline{\alpha(x)} \alpha(x) \beta(x) \overline{\beta(x)}\right) \\
& \left.-\left(q^{2}+q\right) \sum_{x \in \mathbb{F}_{q}^{*}} \overline{\alpha(x)} \alpha(x) \beta(x) \overline{\beta(x)}+(q-1)(q-1)(q+1)+(q+1)^{2}(q-1)\right] \\
& =\frac{1}{q(q-1)^{2}(q+1)}\left[\left(q^{2}+q\right)(q-1)(q-2)+\left(q^{2}+q\right)\left(\sum_{x \in \mathbb{F}_{q}^{*}} \alpha(x) \overline{\beta(x)}\right)\left(\sum_{x \in \mathbb{F}_{q}^{*}} \overline{\alpha(x)} \beta(x)\right)\right. \\
& \left.-\left(q^{2}+q\right)(q-1)+(q-1)^{2}(q+1)+(q+1)^{2}(q-1)\right] .
\end{aligned}
$$

After applying the lemma, this reduces to

$$
\frac{(q-1)(q+1)}{q(q-1)^{2}(q+1)}\left[q^{2}-2 q-q+q-1+q+1\right]=\frac{1}{q(q-1)}\left(q^{2}-q\right)=1
$$

This shows that $W_{\alpha, \beta}$ is irreducible. We conclude that $\operatorname{GL}\left(2, \mathbb{F}_{q}\right)$ has $\frac{(q-1)(q-2)}{2}$ mutually non-isomorphic irreducible representations of the form $W_{\alpha, \beta}$. 


\subsection{The Representation $X_{\phi}$}

Let $\phi: \mathbb{F}_{q^{2}}^{*} \longrightarrow \mathbb{C}^{*}$ be a character, such that $\phi \neq \phi^{q}$. The goal of this section is to construct a new irreducible representation $X_{\phi}$ associated to $\phi$. For two such characters $\phi$ and $\psi$, we have $X_{\phi} \cong X_{\psi}$ exactly when

$$
\phi=\psi, \quad \text { or } \quad \phi=\psi^{q} \quad \text { or } \quad \phi^{q}=\psi
$$

Thus we get $\frac{\left(q^{2}-1\right)-(q-1)}{2}=\frac{1}{2}\left(q^{2}-q\right)$ number of representations of this type. This will complete the list of irreducible $G$-representations. The construction of $X_{\phi}$ is somewhat indirect.

\subsection{1}

Recall that in the previous chapter we have fixed a non-square element $\epsilon \in \mathbb{F}_{q}$. We identify the field $\mathbb{F}_{q^{2}}$ with $\mathbb{F}_{q}[t] /\left(t^{2}-\epsilon\right)$. We have defined the element

$$
d_{x, y}=\left(\begin{array}{ll}
x & \epsilon y \\
y & x
\end{array}\right)
$$

in $G=\operatorname{GL}\left(2, \mathbb{F}_{q}\right)$. Let

$$
K=\left\{d_{x, y}: x, y \in \mathbb{F}_{q} \text { such that } x, y \text { are not both zero }\right\}
$$

Lemma 4.7. The set $K \subseteq G$ is a subgroup.

PROOF. By direct calculation, we can check the equality $d_{x_{1}, y_{1}} d_{x_{2}, y_{2}}=d_{x_{3}, y_{3}}$, where

$$
x_{3}=x_{1} x_{2}+\epsilon y_{1} y_{2}, \quad y_{3}=x_{1} y_{2}+x_{2} y_{1} .
$$


Moreover, $d_{x, y}^{-1}=d_{x^{\prime}, y^{\prime}}$ where

$$
x^{\prime}=\frac{x}{x^{2}-\epsilon y^{2}}, \quad y^{\prime}=\frac{-y}{x^{2}-\epsilon y^{2}} .
$$

This proves the lemma.

Now consider the map

$$
K \stackrel{f}{\longrightarrow} \mathbb{F}_{q^{2}}^{*}, \quad f\left(d_{x, y}\right)=x+t y .
$$

Lemma 4.8. The map $f$ is an isomorphism.

PROOF. It is straightforward to check that

$$
f\left(d_{x_{1}, y_{1}}\right) f\left(d_{x_{2}, y_{2}}\right)=f\left(d_{x_{3}, y_{3}}\right),
$$

with $x_{3}, y_{3}$ as above, hence $f$ is a group homomorphism. Moreover, it is injective and surjective by construction.

Henceforth we identify $K$ with $\mathbb{F}_{q^{2}}^{*}$ via $f$. Let

$$
\phi: \mathbb{F}_{q^{2}}^{*}(\cong K) \rightarrow \mathbb{C}^{*}
$$

be a character and let $Y_{\phi}$ be the corresponding induced representation of $\operatorname{GL}\left(2, \mathbb{F}_{q}\right)$. We have $|K|=q^{2}-1$, and hence

$$
\operatorname{dim} Y_{\phi}=[G: K]=\frac{q(q+1)(q-1)^{2}}{q^{2}-1}=q(q-1)
$$




\subsection{2}

Let $\alpha=\left.\phi\right|_{\mathbb{F}_{q}^{*}}$ be the restriction of $\phi$. This is a character of $\mathbb{F}_{q}^{*}$. When $X_{\phi}$ is constructed, it will be the case that

$$
V \otimes W_{\alpha, 1} \cong W_{\alpha, 1} \oplus Y_{\phi} \oplus X_{\phi}
$$

Let $E_{1}, \ldots, E_{m}$ be all the mutually non-isomorphic irreducible representations of $G$. We may assume $E_{1}=W_{\alpha, 1}$. Then we have isomorphisms

$$
V \otimes W_{\alpha, 1} \cong \bigoplus_{i=1}^{m} E_{i}^{a_{i}}, \quad Y_{\phi} \cong \bigoplus_{i=1}^{m} E_{i}^{b_{i}},
$$

for some nonnegative integers $a_{i}, b_{i}$. Using inner products of characters, we will prove the following proposition.

Proposition 4.9. With notation as above, we have equalities

1. $\sum_{i=1}^{m} a_{i}^{2}=q+3$.
2. $\sum_{i=1}^{m} b_{i}^{2}=q-1$.

3. $a_{1}=2$.

4. $b_{1}=1$.

5. $\sum_{i=1}^{m} a_{i} b_{i}=q$.

Let us tentatively assume the proposition. Then

$$
\begin{aligned}
\sum_{i=2}^{m}\left(a_{i}-b_{i}\right)^{2} & =\sum_{i=2}^{m} a_{i}^{2}+\sum_{i=2}^{m} b_{i}^{2}-2 \sum_{i=2}^{m} a_{i} b_{i} \\
& =(q+3-4)+(q-2)-2(q-2)=1 .
\end{aligned}
$$


Hence, after renumbering $E_{2}, \ldots, E_{m}$ if necessary, we can assume that $a_{i}=b_{i}$ for $2 \leqslant i \leqslant m-1$ and $a_{m}-b_{m}= \pm 1$. Using (4.2), we have

$$
\underbrace{\operatorname{dim}\left(V \otimes W_{\alpha, 1}\right)-\operatorname{dim} Y_{\phi}-\operatorname{dim} E_{1}}_{(\sharp)}=\left(a_{m}-b_{m}\right) \operatorname{dim} E_{m} .
$$

Since

$$
(\sharp)=q(q+1)-q(q-1)-(q+1)=q-1>0,
$$

we deduce that $a_{m}-b_{m}=1$ and $\operatorname{dim} E_{m}=q-1$. Then we set $X_{\phi}=E_{m}$. This also establishes the isomorphism (4.1).

The rest of this section is devoted to proving Proposition 4.9 .

\subsubsection{The character of $Y_{\phi}$}

We will use Proposition 2.30 to find the character values of $Y_{\phi}$. The statements below about conjugacy classes and stabilizers follow from Propositions 3.2 and 3.3 .

We have $C_{G}\left(c_{x, y}\right) \cap K=\emptyset$, hence $\chi_{Y_{\phi}}\left(c_{x, y}\right)=0$.

Also, $C_{G}\left(a_{x}\right) \cap K=C_{K}\left(a_{x}\right)$. By Proposition $3.2(\mathrm{i})$, we get

$$
\left|G_{a_{x}}\right|=|G|=q(q-1)^{2}(q+1), \quad \text { and } \quad\left|K_{a_{x}}\right|=|K|=q^{2}-1 .
$$

Hence

$$
\chi_{Y_{\phi}}\left(a_{x}\right)=\left|G_{a_{x}}\right|\left(\frac{\phi(x)}{\left|K_{a_{x}}\right|}\right)=q(q-1)^{2}(q+1)\left(\frac{\phi(x)}{q^{2}-1}\right)=q(q-1) \phi(x) .
$$

Now $C_{G}\left(b_{x}\right) \bigcap K=\emptyset$, hence $\chi_{Y_{\phi}}\left(b_{x}\right)=0$. Finally, $C_{G}\left(d_{x, y}\right) \cap K=C_{K}\left(d_{x, y}\right) \cup C_{K}\left(d_{x,-y}\right)$.

Let $w=x+y t=x+y \sqrt{\epsilon}$. We will write $\tilde{w}=x-y t=x-y \sqrt{\epsilon}$. By Proposition 3.4(i),

$$
\left|G_{d_{x, y}}\right|=\left|K_{d_{x, y}}\right|=\left|K_{d_{x,-y}}\right|=(q-1)(q+1) .
$$


Therefore,

$$
\chi_{Y_{\phi}}\left(d_{x, y}\right)=(q-1)(q+1)\left(\frac{\phi(w)}{(q-1)(q+1)}+\frac{\phi(\tilde{w})}{(q-1)(q+1)}\right)=\phi(w)+\phi(\tilde{w})
$$

In conclusion, the character table of $Y_{\phi}$ is as follows:

\begin{tabular}{|c||c|c|c|c|}
\hline & $a_{x}$ & $b_{x}$ & $c_{x, y}$ & $d_{x, y}$ \\
\hline$\chi_{Y_{\phi}}$ & $q(q-1) \phi(x)$ & 0 & 0 & $\phi(w)+\phi(\tilde{w})$ \\
\hline
\end{tabular}

We will compare this with the character corresponding to $\phi^{q}$. First, note that $\left.\phi^{q}\right|_{\mathbb{F}_{q}^{*}}=\alpha^{q}=\alpha$, since $\alpha$ is a character of the group $\mathbb{F}_{q}^{*}$ of order $q-1$.

Moreover, note that $\tilde{w}=w^{q}$ is the conjugate of $w$ over the $\mathbb{F}_{q}$. Then

$$
\phi^{q}(w)+\phi^{q}(\tilde{w})=\phi^{q}(w)+\phi^{q}\left(w^{q}\right)=\phi(\tilde{w})+\phi^{q^{2}}(w)=\phi(\tilde{w})+\phi(w)
$$

and hence the character of $Y_{\phi^{q}}$ is the same as that of $Y_{\phi}$. This implies $X_{\phi} \cong X_{\phi^{q}}$. 
We have $\left\|\chi_{Y_{\phi}}\right\|^{2}=\sum_{i=1}^{m} b_{i}^{2}$. Using the table above,

$$
\begin{aligned}
& \left\|\chi_{Y_{\phi}}\right\|^{2} \\
& =\frac{1}{|G|}\left[\sum_{x \in \mathbb{F}_{q}^{*}}(q(q-1) \phi(x))(\overline{q(q-1) \phi(x)})+\left(\frac{q(q-1)}{2}\right) \sum_{w \in \mathbb{F}_{q^{*}} \backslash \mathbb{F}_{q}^{*}}\left(\phi(w)+\phi\left(w^{q}\right)\right)\left(\overline{\phi(w)+\phi\left(w^{q}\right)}\right)\right] \\
& =\frac{1}{q(q-1)^{2}(q+1)}\left[(q-1)^{2} q^{2} \sum_{x \in \mathbb{F}_{q}^{*}} 1+\left(\frac{q(q-1)}{2}\right) \sum_{w \in \mathbb{F}_{q^{2}}^{*} \backslash \mathbb{F}_{q}^{*}}\left(1+\phi(w) \overline{\phi\left(w^{q}\right)}+\phi\left(w^{q}\right) \overline{\phi(w)}+1\right)\right] \\
& =\frac{1}{q(q-1)^{2}(q+1)}\left[(q-1)^{2} q^{2}(q-1)+\left(\frac{q(q-1)}{2}\right) \cdot\left(\sum_{w \in \mathbb{F}_{q^{2}}^{*} \backslash \mathbb{F}_{q}^{*}} \phi(w) \overline{\phi^{q}(w)}+\sum_{x \in \mathbb{F}_{q}^{*}} \phi(x) \overline{\phi^{q}(x)}\right.\right. \\
& \left.+\sum_{w \in \mathbb{F}_{q^{2}}^{*} \backslash \mathbb{F}_{q}^{*}} 2+\sum_{w \in \mathbb{F}_{q^{2}}^{*} \backslash \mathbb{F}_{q}^{*}} \phi^{q}(w) \overline{\phi(w)}+\sum_{x \in \mathbb{F}_{q}^{*}} \phi^{q}(x) \overline{\phi(x)}\right)-\frac{q(q-1)}{2} \sum_{x \in \mathbb{F}_{q}^{*}} \phi^{q}(x) \overline{\phi(x)} \\
& \left.-\frac{q(q-1)}{2} \sum_{x \in \mathbb{F}_{q}^{*}} \phi(x) \overline{\phi^{q}(x)}\right] \\
& =\frac{1}{q(q-1)^{2}(q+1)}\left[(q-1)^{2} q^{2}(q-1)+\left(\frac{q(q-1)}{2}\right) \cdot\left(\sum_{w \in \mathbb{F}_{q^{*}}^{*}} \phi(w) \overline{\phi^{q}(w)}\right.\right. \\
& \left.\left.+2 q(q-1)+\sum_{w \in \mathbb{F}_{q^{2}}^{*}} \phi^{q}(w) \overline{\phi(w)}\right)-\frac{q(q-1)}{2} \sum_{x \in \mathbb{F}_{q}^{*}} \phi(x) \overline{\phi(x)}-\frac{q(q-1)}{2} \sum_{x \in \mathbb{F}_{q}^{*}} \phi(x) \overline{\phi(x)}\right] \\
& =\frac{1}{q(q-1)^{2}(q+1)}\left[(q-1)^{3} q^{2}+q^{2}(q-1)^{2}-\frac{q(q-1)}{2}(q-1)-\frac{q(q-1)}{2}(q-1)\right] \\
& =\frac{1}{q(q-1)^{2}(q+1)}\left[(q-1)^{3} q^{2}+q^{2}(q-1)^{2}-q(q-1)^{2}\right] \\
& =\frac{q(q-1)^{2}}{q(q-1)^{2}(q+1)}\left[q^{2}-q+q-1\right]=q-1 \text {. }
\end{aligned}
$$

Here we have used the fact that, since $\phi^{q} \neq \phi$, we have $\sum_{w \in \mathbb{F}_{q^{*}}} \phi^{q}(w) \overline{\phi(w)}=0$ by Lemma 4.6. This proves part (2) of Proposition 4.9. 


\subsection{4}

The character table of $V \otimes W_{\alpha, 1}$ is as follows.

\begin{tabular}{|c||c|c|c|c|}
\hline & $a_{x}$ & $b_{x}$ & $c_{x, y}$ & $d_{x, y}$ \\
\hline \hline$\chi_{V \otimes W_{\alpha, 1}}$ & $q(q+1) \alpha(x)$ & 0 & $\alpha(x)+\alpha(y)$ & 0 \\
\hline
\end{tabular}

We have

$$
\left\langle\chi_{V \otimes W_{\alpha, 1}}, \chi_{V \otimes W_{\alpha, 1}}\right\rangle=\sum_{i=1}^{m} a_{i}^{2}
$$

Using the table,

$$
\begin{aligned}
& \left\|\chi_{V \otimes W_{\alpha, 1}}\right\|^{2} \\
= & \frac{1}{|G|}\left[\sum_{x \in \mathbb{F}_{q}^{*}}\left(q^{2}(q+1)^{2} \alpha(x) \overline{\alpha(x)}+\left(\frac{q^{2}+q}{2}\right) \sum_{x, y \in \mathbb{F}_{q}^{*}, x \neq y}(\alpha(x)+\alpha(y)) \overline{(\alpha(x)+\alpha(y))}\right]\right. \\
= & \frac{1}{q(q-1)^{2}(q+1)}\left[\sum_{x \in \mathbb{F}_{q}^{*}} q^{2}(q+1)^{2} \cdot 1+\frac{q^{2}+q}{2}\left(\sum_{x, y \in \mathbb{F}_{q}^{*}, x \neq y}[\alpha(x) \overline{\alpha(x)}+\alpha(x) \overline{\alpha(y)}\right.\right. \\
+ & \alpha(y) \overline{\alpha(x)}+\alpha(y) \overline{\alpha(y)}])] \\
= & \frac{1}{q(q-1)^{2}(q+1)}\left[q^{2}(q+1)^{2}(q-1)+\frac{q^{2}+q}{2}\left(2(q-1)(q-2)+\sum_{x, y \in \mathbb{F}_{q}^{*}, x \neq y} \alpha(x) \overline{\alpha(y)}\right.\right. \\
& \left.+\sum_{x \in \mathbb{F}_{q}^{*}} \alpha(x) \overline{\alpha(x)}+\sum_{x, y \in \mathbb{F}_{q}^{*}, x \neq y} \alpha(y) \overline{\alpha(x)}+\sum_{x \in \mathbb{F}_{q}^{*}} \alpha(x) \overline{\alpha(x)}-2 \sum_{x \in \mathbb{F}_{q}^{*}} \alpha(x) \overline{\alpha(x)}\right] \\
= & \frac{1}{q(q-1)^{2}(q+1)}\left[q^{2}(q+1)^{2}(q-1)+q(q-1)(q-2)(q+1)+\right. \\
& \left.\frac{q^{2}+q}{2}\left(\sum_{x \in \mathbb{F}_{q}^{*}} \alpha(x)\right)\left(\sum_{y \in \mathbb{F}_{q}^{*}} \overline{\alpha(y)}\right)+\frac{q^{2}+q}{2}\left(\sum_{y \in \mathbb{F}_{q}^{*}} \alpha(y)\right)\left(\sum_{x \in \mathbb{F}_{q}^{*}} \overline{\alpha(x)}\right)-q(q-1)(q+1)\right] \\
= & \frac{q(q-1)(q+1)}{q(q-1)^{2}(q+1)}\left[\left(q^{2}+q\right)+(q-2)-1\right]=\frac{1}{(q-1)}(q+3)(q-1)=q+3 .
\end{aligned}
$$

Here we have used the fact that $\sum_{x \in \mathbb{F}_{q}^{*}} \alpha(x)=0$, since $\alpha \neq 1$. This proves part (1) of Proposition 4.9 


\subsection{5}

Next we calculate

$$
\left\langle\chi_{V \otimes W_{\alpha, 1}}, \chi_{W_{\alpha, 1}}\right\rangle=a_{1}
$$

We have

$$
\begin{aligned}
& \left\langle\chi_{V \otimes W_{\alpha, 1}}, \chi_{W_{\alpha, 1}}\right\rangle \\
& =\frac{1}{|G|}\left[\sum _ { a _ { x } } \left(q(q+1)^{2} \alpha(x) \overline{\alpha(x)}+\left(\frac{q^{2}+q}{2}\right) \sum_{c_{x, y}}(\alpha(x)+\alpha(y) \overline{(\alpha(x)+\alpha(y))}]\right.\right. \\
& =\frac{1}{q(q-1)^{2}(q+1)}\left[\sum_{x \in \mathbb{F}_{q}^{*}} q(q+1)^{2} \cdot 1+\frac{q^{2}+q}{2}\left(\sum_{x, y \in \mathbb{F}_{q}^{*}, x \neq y}[\alpha(x) \overline{\alpha(x)}+\alpha(x) \overline{\alpha(y)}\right.\right. \\
& +\alpha(y) \overline{\alpha(x)}+\alpha(y) \overline{\alpha(y)}])] \\
& =\frac{1}{q(q-1)^{2}(q+1)}\left[q^{2}(q+1)^{2}(q-1)+\frac{q^{2}+q}{2}\left((2 q-2)(q-2)+\sum_{\substack{x, y \in \mathbb{F}_{q}^{*} \\
x \neq y}} \alpha(x) \overline{\alpha(y)}\right.\right. \\
& \left.\left.+\sum_{x \in \mathbb{F}_{q}^{*}} \alpha(x) \overline{\alpha(x)}+\sum_{\substack{x, y \in \mathbb{F}_{q}^{*} \\
x \neq y}} \alpha(y) \overline{\alpha(x)}+\sum_{x \in \mathbb{F}_{q}^{*}} \alpha(x) \overline{\alpha(x)}-2 \sum_{x \in \mathbb{F}_{q}^{*}} \alpha(x) \overline{\alpha(x)}\right)\right] \\
& =\frac{1}{q(q-1)^{2}(q+1)}\left[q(q+1)^{2}(q-1)+q(q-1)(q-2)(q+1)\right. \\
& +\frac{q^{2}+q}{2}\left(\sum_{x \in \mathbb{F}_{q}^{*}} \alpha(x)\right)\left(\sum_{y \in \mathbb{F}_{q}^{*}} \overline{\alpha(y)}\right) \\
& \left.+\frac{q^{2}+q}{2}\left(\sum_{y \in \mathbb{F}_{q}^{*}} \alpha(y)\right)\left(\sum_{x \in \mathbb{F}_{q}^{*}} \overline{\alpha(x)}\right)-q(q-1)(q+1)\right] \\
& =\frac{q(q-1)(q+1)}{q(q-1)^{2}(q+1)}[(q+1)+(q-2)-1]=\frac{1}{(q-1)} 2(q-1)=2 .
\end{aligned}
$$

This proves part (3) of Proposition 4.9.

Next we calculate

$$
b_{1}=\left\langle\chi_{Y_{\phi}}, \chi_{W_{\alpha, 1}}\right\rangle=\frac{1}{|G|}(q-1) q(q+1) \sum_{x \in \mathbb{F}_{q}^{*}} \phi(x) \overline{\alpha(x)}=1,
$$


which proves part (4) of Proposition 4.9.

\subsection{6}

Finally, we have

$$
\sum_{i=1}^{m} a_{i} b_{i}=\left\langle\chi_{V \otimes W_{\alpha, 1}}, \chi_{Y_{\phi}}\right\rangle=\frac{1}{|G|} q^{2}(q-1)(q+1) \sum_{x \in \mathbb{F}_{q}^{*}} \phi(x) \overline{\alpha(x)}=q
$$

which proves part (5) of Proposition 4.9. This completes the proof of the proposition, and hence the construction of $X_{\phi}$. We have seen that $X_{\phi} \cong X_{\phi^{q}}$. Using inner product of characters as above, we can show that if $\psi$ is another character such that $\psi \neq \phi, \phi^{q}$, then $\left\langle\chi_{X_{\phi}}, \chi_{X_{\psi}}\right\rangle=0$ implying that $X_{\phi} \nRightarrow X_{\psi}$. We omit this calculation for brevity.

Since

$$
\chi_{X_{\phi}}=\chi_{V \otimes W_{\alpha, 1}}-\chi_{W_{\alpha, 1}}-\chi_{Y_{\phi}}
$$

we get the following character table:

\begin{tabular}{|c||c|c|c|c|}
\hline & $a_{x}$ & $b_{x}$ & $c_{x, y}$ & $d_{x, y}$ \\
\hline \hline$\chi_{X_{\phi}}$ & $(q-1) \phi(x)$ & $-\phi(x)$ & 0 & $-\left(\phi(w)+\phi\left(w^{q}\right)\right)$ \\
\hline
\end{tabular}

Note that there are $q^{2}-1$ characters $\phi: \mathbb{F}_{q^{2}}^{*} \longrightarrow \mathbb{C}^{*}$, out of which $q-1$ satisfy the condition $\phi^{q}=\phi$. Moreover, $\phi$ and $\phi^{q}$ lead to the same representation. Hence there are $\frac{\left(q^{2}-1\right)-(q-1)}{2}=$ $\frac{1}{2}\left(q^{2}-q\right)$ mutually non-isomorphic representations of type $X_{\phi}$. The total number of representations $U_{\alpha}, V_{\alpha}, W_{\alpha, \beta}, X_{\phi}$ constructed so far is

$$
(q-1)+(q-1)+\frac{(q-1)(q-2)}{2}+\frac{q^{2}-q}{2}=q^{2}-1
$$

which is the same as the number of conjugacy classes in $\operatorname{GL}\left(2, \mathbb{F}_{q}\right)$. Hence we have a complete list 
of irreducible representations.

\subsection{The Character Table of $\mathbf{G L}\left(2, \mathbb{F}_{q}\right)$}

We have constructed four types of mutually non-isomorphic irreducible representations of $G$, which give a total $q^{2}-1$ irreducible representations. In summary,

- There are $q-1$ representations $U_{\alpha}$ each of dimension 1 .

- There are $q-1$ representations $V_{\alpha}$ each of dimension $q$.

- There are $\frac{(q-1)(q-2)}{2}$ representations $W_{\alpha, \beta}$ each of dimension $q+1$.

- There are $\frac{q^{2}-q}{2}$ representations $X_{\phi}$ each of dimension $q-1$.

\subsection{1}

Recall that by Proposition 2.18, if $E_{1}, \ldots, E_{m}$ are all the pairwise non-isomorphic irreducible representations of a group $G$, then

$$
|G|=\sum_{i=1}^{m}\left(\operatorname{dim} E_{i}\right)^{2} .
$$

Using the data above, we check that

$(q-1) \cdot 1^{2}+(q-1) q^{2}+\frac{(q-1)(q-2)}{2}(q+1)^{2}+\frac{q^{2}-q}{2}(q-1)^{2}=q(q+1)(q-1)^{2}=\left|\mathrm{GL}\left(2, \mathbb{F}_{q}\right)\right|$ 
The entire character table of $\mathrm{GL}\left(2, \mathbb{F}_{q}\right)$ is as follows:

\begin{tabular}{|c||c|c|c|c|}
\hline & $a_{x}$ & $b_{x}$ & $c_{x, y}$ & $d_{x, y}$ \\
\hline \hline class size & 1 & $q^{2}-1$ & $q^{2}+q$ & $q^{2}-q$ \\
\hline \hline$\chi_{U_{\alpha}}$ & $\alpha\left(x^{2}\right)$ & $\alpha\left(x^{2}\right)$ & $\alpha(x y)$ & $\alpha\left(w^{q+1}\right)$ \\
\hline$\chi_{V_{\alpha}}$ & $q \alpha\left(x^{2}\right)$ & 0 & $\alpha(x y)$ & $-\alpha\left(w^{q+1}\right)$ \\
\hline$\chi_{W_{\alpha, \beta}}$ & $(q+1) \alpha(x) \beta(x)$ & $\alpha(x) \beta(x)$ & $\alpha(x) \beta(y)+\alpha(y) \beta(x)$ & 0 \\
\hline$\chi_{X_{\phi}}$ & $(q-1) \phi(x)$ & $-\phi(x)$ & 0 & $-\left(\phi(w)+\phi\left(w^{q}\right)\right)$ \\
\hline
\end{tabular}

This table will be useful for calculating the Clebsch-Gordan decompositions in the next chapter.

\subsubsection{Dual representations}

From the character table, it is straightforward to identify the dual of each irreducible representation (see Section 2.8.1). We have isomorphisms

$$
\left(U_{\alpha}\right)^{\vee} \cong U_{\alpha^{-1}}, \quad\left(V_{\alpha}\right)^{\vee} \cong V_{\alpha^{-1}}, \quad\left(W_{\alpha, \beta}\right)^{\vee} \cong W_{\alpha^{-1}, \beta^{-1}}, \quad\left(X_{\phi}\right)^{\vee} \cong X_{\phi^{-1}}
$$

It follows that if $E$ is an irreducible $G$-representation, then $E^{\vee}$ is also irreducible of the same type as $E$. 


\section{Chapter 5}

\section{Clebsch-Gordan Decompositions}

In the previous chapter, we have constructed a total of $q^{2}-1$ irreducible representations of $\mathrm{GL}\left(2, \mathbb{F}_{q}\right)$. It turned out that there are four types of representations:

- For each character $\alpha: \mathbb{F}_{q}^{*} \longrightarrow \mathbb{C}^{*}$, we have the representations $U_{\alpha}$ and $V_{\alpha}=V \otimes U_{\alpha}$.

- For each unordered pair of distinct characters $\alpha, \beta: \mathbb{F}_{q}^{*} \longrightarrow \mathbb{C}$, we have the representation $W_{\alpha, \beta}$.

- For each character $\phi: \mathbb{F}_{q^{2}}^{*} \longrightarrow \mathbb{C}^{*}$ such that $\phi \neq \phi^{q}$, we have the representation $X_{\phi}$. Moreover, $X_{\phi} \cong X_{\phi^{q}}$.

If $E_{1}$ and $E_{2}$ are any two irreducible representations, then their tensor product $E_{1} \otimes E_{2}$ will in general decompose into a direct sum of irreducibles. These are usually called Clebsch-Gordan decompositions, since in the 19th century Clebsch and Gordan studied them in the context of representations of the group $\mathrm{GL}(2, \mathbb{C})$ (see $[9, \mathrm{Ch} .4])$.

The theme of this chapter is to compute the decompositions of the following six tensor products:

$$
V \otimes V, \quad V \otimes W_{\alpha, 1}, \quad V \otimes X_{\phi}, \quad W_{\alpha, 1} \otimes X_{\phi}, \quad X_{\phi} \otimes X_{\psi}, \quad W_{\alpha, 1} \otimes W_{\beta, 1},
$$


where $\alpha, \beta$ denote characters of $\mathbb{F}_{q}^{*}$, and $\phi, \psi$ denote characters of $\mathbb{F}_{q^{2}}^{*}$. Moreover, if $E$ is an arbitrary irreducible representation, then we give precise formulae for the representation $E \otimes U_{\alpha}$ which is also irreducible.

All the general Clebsch-Gordan decompositions follow from these computations. For example, we can rewrite $V_{\alpha} \otimes V_{\beta}$ as $(V \otimes V) \otimes U_{\alpha \beta}$ and then decompose it using the results above.

We mention some preliminary results in the next section. In section 5.2, we discuss all representations of the type $E \otimes U_{\alpha}$, where $E$ is an arbitrary irreducible representation. The six calculations listed in $(\dagger)$ are given in Sections 5.3 through 5.8 . They are an original contribution of this thesis in the sense that we haven't found them in the existing literature.

\subsection{Preliminary Results}

Recall that we have fixed an element $\epsilon \in \mathbb{F}_{q}$, such that $t^{2}-\epsilon$ is an irreducible polynomial in $\mathbb{F}_{q}[t]$. In other words, $\epsilon$ is not the square of any element in $\mathbb{F}_{q}$. We have identified the field $\mathbb{F}_{q^{2}}$ with $\mathbb{F}_{q}[t] /\left(t^{2}-\epsilon\right)$, and $t$ with the symbol $\sqrt{\epsilon}$. Each element in $\mathbb{F}_{q^{2}}$ can be written as $x+y \sqrt{\epsilon}$ for unique elements $x, y \in \mathbb{F}_{q}$.

The conjugate of $w=x+y \sqrt{\epsilon}$ over $\mathbb{F}_{q}$ is $w^{q}=x-y \sqrt{\epsilon}$. Then

$$
w^{q+1}=w w^{q}=x^{2}-\epsilon y^{2} \in \mathbb{F}_{q}
$$

Define the norm map

$$
\nu: \mathbb{F}_{q^{2}}^{*} \longrightarrow \mathbb{F}_{q}^{*}, \quad \nu(w)=w^{q+1}
$$

Lemma 5.1. The map $\nu$ is a surjective group homomorphism.

PROOF. It is clear from the definition that $\nu$ is a homomorphism. We have $\operatorname{ker}(\nu)=\left\{w \in \mathbb{F}_{q}\right.$ : 
$\left.w^{q+1}=1\right\}$, and hence $|\operatorname{ker}(\nu)|=q+1$. This implies that

$$
|\operatorname{Im}(\nu)|=\frac{q^{2}-1}{q+1}=q-1=\left|\mathbb{F}_{q}^{*}\right| .
$$

Hence $\nu$ is surjective.

Let $\alpha: \mathbb{F}_{q}^{*} \longrightarrow \mathbb{C}^{*}$ be a character. Then $\widetilde{\alpha}=\alpha \circ \nu$ is a character $\mathbb{F}_{q^{2}}^{*} \longrightarrow \mathbb{C}^{*}$. Since $\nu$ is surjective, we know that if $\alpha$ is non-trivial, then so is $\widetilde{\alpha}$.

\subsection{1}

We have an inclusion of cyclic groups $\mathbb{F}_{q}^{*} \subseteq \mathbb{F}_{q^{2}}^{*}$. We fix a cyclic generator $s$ for $\mathbb{F}_{q^{2}}^{*}$, i.e., $\mathbb{F}_{q^{2}}^{*}=\langle s\rangle$. Then $\mathbb{F}_{q}^{*}=\langle r\rangle$, where $r=s^{q+1}$.

A character $\phi: \mathbb{F}_{q^{2}}^{*} \longrightarrow \mathbb{C}^{*}$ is completely given by an integer $k$ (determined modulo $\left.q^{2}-1\right)$ such that

$$
\phi(s)=\exp \left(\frac{2 \pi \iota k}{q^{2}-1}\right)
$$

Then $\phi^{q} \neq \phi$ translates into the condition that $k$ is not divisible by $q+1$. Similarly, a character $\alpha: \mathbb{F}_{q}^{*} \longrightarrow \mathbb{C}^{*}$ is specified by an integer $k$ (determined modulo $q-1$ ) such that

$$
\alpha(r)=\exp \left(\frac{2 \pi \iota k}{q-1}\right) .
$$

\subsection{2}

If $E$ is an irreducible $G$-representation and $E^{\prime}$ is any representation, we write $m\left(E, E^{\prime}\right)$ for the multiplicity of $E$ in the irreducible decomposition of $E^{\prime}$. By Proposition 2.23, we have

$$
m\left(E, E^{\prime}\right)=\left\langle\chi_{E}, \chi_{E^{\prime}}\right\rangle .
$$


As usual, let $U_{\mathbb{T}}$ denote the one-dimensional trivial representation. We will repeatedly use the following lemma in our calculations.

Lemma 5.2. Let $E_{1}, E_{2}, E_{3}$ be irreducible representations. Then we have

$$
m\left(E_{1}^{\vee}, E_{2} \otimes E_{3}\right)=m\left(E_{2}^{\vee}, E_{1} \otimes E_{3}\right)=m\left(E_{3}^{\vee}, E_{1} \otimes E_{2}\right) .
$$

PROOF. This follows because each number is equal to

$$
m\left(U_{\mathbb{T}}, E_{1} \otimes E_{2} \otimes E_{3}\right)=\frac{1}{|G|} \sum_{g \in G} \chi_{E_{1}}(g) \chi_{E_{2}}(g) \chi_{E_{3}}(g)
$$

The advantage of the lemma is that it reduces the number of calculations. For example, suppose that we know the number of copies of $X_{\phi}$ in $V_{\alpha} \otimes V_{\beta}$, for any characters $\alpha, \beta$ of $\mathbb{F}_{q}^{*}$ and $\phi$ of $\mathbb{F}_{q^{2}}^{*}$. Then the lemma allows us to switch between representations of types $V$ and $X$. Using the isomorphisms in Section 4.7.2, we can then deduce the number of copies of $V_{\beta^{\prime}}$ in $X_{\phi^{\prime}} \otimes V_{\alpha^{\prime}}$ for arbitrary $\alpha^{\prime}, \beta^{\prime}, \phi^{\prime}$.

\subsection{Tensor Products with $U_{\alpha}$}

In this section, we will discuss the tensor products of all irreducible representations with the onedimensional representation $U_{\alpha}$. We will use the character table given in the previous chapter. Recall that we have elements

$$
a_{x}=\left(\begin{array}{cc}
x & 0 \\
0 & x
\end{array}\right), \quad b_{x}=\left(\begin{array}{ll}
x & 1 \\
0 & x
\end{array}\right), \quad c_{x, y}=\left(\begin{array}{ll}
x & 0 \\
0 & y
\end{array}\right), \quad d_{x, y}=\left(\begin{array}{ll}
x & \epsilon y \\
y & x
\end{array}\right)
$$


which represent conjugacy classes in $G=\operatorname{GL}\left(2, \mathbb{F}_{q}\right)$. Let $w=x+y \sqrt{\epsilon} \in \mathbb{F}_{q^{2}}$ denote the element corresponding to $d_{x, y}$.

Let $\alpha, \beta$ and $\gamma$ denote characters of $\mathbb{F}_{q}^{*}$. We have the following character table:

\begin{tabular}{|c||c|c|c|c|}
\hline & $a_{x}$ & $b_{x}$ & $c_{x, y}$ & $d_{x, y}$ \\
\hline \hline$U_{\alpha}$ & $\alpha\left(x^{2}\right)$ & $\alpha\left(x^{2}\right)$ & $\alpha(x y)$ & $\alpha\left(w^{q+1}\right)$ \\
\hline$V_{\alpha}$ & $q \alpha\left(x^{2}\right)$ & 0 & $\alpha(x y)$ & $-\alpha\left(w^{q+1}\right)$ \\
\hline$W_{\alpha, \beta}$ & $(q+1) \alpha(x) \beta(x)$ & $\alpha(x) \beta(x)$ & $\alpha(x) \beta(y)+\alpha(y) \beta(x)$ & 0 \\
\hline$X_{\phi}$ & $(q-1) \phi(x)$ & $-\phi(x)$ & 0 & $-\left(\phi(w)+\phi\left(w^{q}\right)\right)$ \\
\hline$U_{\alpha} \otimes U_{\beta}$ & $\alpha\left(x^{2}\right) \beta\left(x^{2}\right)$ & $\alpha\left(x^{2}\right) \beta\left(x^{2}\right)$ & $\alpha(x y) \beta(x y)$ & $\alpha\left(w^{q+1}\right) \beta\left(w^{q+1}\right)$ \\
\hline$V_{\alpha} \otimes U_{\beta}$ & $q \alpha\left(x^{2}\right) \beta\left(x^{2}\right)$ & 0 & $\alpha(x y) \beta(x y)$ & $-\alpha\left(w^{q+1}\right) \beta\left(w^{q+1}\right)$ \\
\hline$W_{\alpha, \beta} \otimes U_{\gamma}$ & $(q+1)[\alpha(x) \gamma(x)$ & $\alpha(x) \gamma(x)+$ & $\alpha(x) \gamma(x) \beta(y) \gamma(y)+$ & 0 \\
\hline$X_{\phi} \otimes U_{\alpha}$ & $(q-1) \phi(x) \alpha(\nu(x))$ & $-\phi(x) \alpha(\nu(x))$ & $\alpha(y) \gamma(y) \beta(x) \gamma(x)$ & \\
\hline
\end{tabular}

We know that two representations are isomorphic, if and only if they have same character values on each conjugacy class. This gives the following isomorphisms:

$$
\begin{array}{ll}
U_{\alpha} \otimes U_{\beta} \cong U_{\alpha \beta}, & V_{\alpha} \otimes U_{\beta} \cong V_{\alpha \beta}, \\
W_{\alpha, \beta} \otimes U_{\gamma} \cong W_{\alpha \gamma, \beta \gamma}, & X_{\phi} \otimes U_{\alpha} \cong X_{\phi \widetilde{\alpha}} .
\end{array}
$$

It follows that if $E$ is any irreducible representation of $G$, then $E \otimes U_{\alpha}$ is also irreducible of the same type. Hence we deduce the following statement:

Lemma 5.3. If $E_{1}, E_{2}$ are of different types, then $U_{\alpha}$ is not a summand in the decomposition of $E_{1} \otimes E_{2}$

PROOF. By Lemma 5.2, if $U_{\alpha}$ is a summand then $E_{1}^{\vee}$ would be a summand in $E_{2} \otimes U_{\alpha}$, which is impossible. 
For example, $U_{\alpha}$ cannot be a summand in $V_{\beta} \otimes X_{\phi}$.

\subsection{The Decomposition of $V \otimes V$}

Recall the definition of the irreducible representation $V$ from Section 4.3. For each type of irreducible representation $E$ of $\operatorname{GL}\left(2, \mathbb{F}_{q}\right)$, we will find the number of copies of $E$ in the decomposition of $V \otimes V$. The final result will be summarized in Proposition 5.4 below. Consider the following character table:

\begin{tabular}{|c||r|r|r|r|}
\hline & $a_{x}$ & $b_{x}$ & $c_{x, y}$ & $d_{x, y}$ \\
\hline \hline$\chi_{V}$ & $q$ & 0 & 1 & -1 \\
\hline$\chi_{V \otimes V}$ & $q^{2}$ & 0 & 1 & 1 \\
\hline
\end{tabular}

Recall that the number of copies of $E$ in $V \otimes V$ is given by the inner product $\left\langle\chi_{V \otimes V}, \chi_{E}\right\rangle$.

\subsection{1}

First, we will find copies of $U_{\alpha}$ in $V \otimes V$, where $\alpha$ is an arbitrary character of $\mathbb{F}_{q}^{*}$. We have

$$
\begin{aligned}
& \mathbb{S}=\left\langle\chi_{V \otimes V}, \chi_{U_{\alpha}}\right\rangle \\
= & \frac{1}{|G|}\left[\sum_{x \in \mathbb{F}_{q}^{*}} q^{2} \alpha\left(x^{2}\right) \cdot 1+0+\frac{1}{2} \sum_{\substack{x, y \in \mathbb{F}_{q}^{*} \\
x \neq y}} \alpha(x) \alpha(y)\left(q^{2}+q\right)+\frac{1}{2} \sum_{\substack{x, y \in \mathbb{F}_{q} \\
y \neq 0}} \alpha\left(x^{2}-\epsilon y^{2}\right)\left(q^{2}-q\right)\right],
\end{aligned}
$$


where the factor $\frac{1}{2}$ appears because $c_{x, y}$ and $c_{y, x}$ are in the same conjugacy class, and similarly for $d_{x, y}$ and $d_{x,-y}$. This expression equals

$$
\begin{aligned}
& \frac{1}{q(q-1)^{2}(q+1)} \sum_{x \in \mathbb{F}_{q}^{*}}\left(\frac{1}{2}\left(q^{2}+q\right)+\frac{1}{2}\left(q^{2}-q\right)\right) \alpha\left(x^{2}\right) .1 \\
& +\frac{1}{2} \sum_{x, y \in \mathbb{F}_{q}^{*}, x \neq y} \alpha(x) \alpha(y)\left(q^{2}+q\right)+\frac{1}{2} \sum_{x, y \in \mathbb{F}_{q}, y \neq 0} \alpha\left(x^{2}-\epsilon y^{2}\right)\left(q^{2}-q\right) \\
= & \frac{1}{|G|}\left[\frac{1}{2}\left(q^{2}+q\right)\left(\sum_{x \in \mathbb{F}_{q}^{*}} \alpha\left(x^{2}\right)+\sum_{x, y \in \mathbb{F}_{q}^{*}, x \neq y} \alpha(x) \alpha(y)\right)\right. \\
& \left.+\frac{1}{2}\left(q^{2}-q\right)\left(\sum_{x \in \mathbb{F}_{q}^{*}} \alpha\left(x^{2}\right)+\sum_{x, y \in \mathbb{F}_{q}, y \neq 0} \alpha\left(x^{2}-\epsilon y^{2}\right)\right)\right] \\
= & \frac{1}{\left.q(q-1)^{2}(q+1)\right)}\left[\frac{1}{2}\left(q^{2}+q\right)\left(\sum_{x \in \mathbb{F}_{q}^{*}} \alpha(x)\right)\left(\sum_{y \in \mathbb{F}_{q}^{*}} \alpha(y)\right)\right. \\
+ & \left.\frac{1}{2}\left(q^{2}-q\right)\left(\sum_{w \in \mathbb{F}_{q^{2}}^{*}} \alpha(\nu(w))\right)\right] .
\end{aligned}
$$

We use Lemma 4.5 to simplify the sums. If $\alpha$ is non-trivial, then $\mathbb{S}=0$. If $\alpha$ is trivial, then

$$
\left.\mathbb{S}=\frac{1}{\left.q(q-1)^{2}(q+1)\right)}\left[\frac{1}{2}\left(q^{2}+q\right)(q-1)(q-1)+\frac{1}{2}\left(q^{2}-q\right)\right)\left(q^{2}-1\right)\right]=1
$$

Hence, there is one copy of the trivial representation $U_{\mathbb{T}}$ inside $V \otimes V$, and no copies of $U_{\alpha}$ if $\alpha$ is non-trivial. 


\section{3 .2}

Now, we will find the copies of $V_{\alpha}$ in $V \otimes V$. We have

$$
\begin{aligned}
\mathbb{S}= & \left\langle\chi_{V \otimes V}, \chi_{V_{\alpha}}\right\rangle \\
= & \frac{1}{|G|}\left[\sum_{x \in \mathbb{F}_{q} *} q^{3} \alpha\left(x^{2}\right) .1+0+\frac{1}{2} \sum_{x, y \in \mathbb{F}_{q}^{*}, x \neq y} \alpha(x) \alpha(y)\left(q^{2}+q\right)-\frac{1}{2} \sum_{x, y \in \mathbb{F}_{q}, y \neq 0} \alpha\left(x^{2}-\epsilon y^{2}\right)\left(q^{2}-q\right)\right] \\
= & \frac{1}{q(q-1)^{2}(q+1)} \sum_{x \in \mathbb{F}_{q^{*}}} q^{3} \alpha\left(x^{2}\right)+\frac{1}{2}\left(q^{2}+q\right) \sum_{x, y \in \mathbb{F}_{q}^{*}, x \neq y} \alpha(x) \alpha(y)+\frac{1}{2}\left(q^{2}+q\right) \sum_{x \in \mathbb{F}_{q} *} \alpha\left(x^{2}\right) \\
& -\frac{1}{2}\left(q^{2}+q\right) \sum_{x \in \mathbb{F}_{q} *} \alpha\left(x^{2}\right)-\frac{1}{2}\left(q^{2}-q\right) \sum_{x, y \in \mathbb{F}_{q}, y \neq 0} \alpha\left(x^{2}-\epsilon y^{2}\right)+\frac{1}{2}\left(q^{2}-q\right) \sum_{x \in \mathbb{F}_{q} *} \alpha\left(x^{2}\right) \\
& -\frac{1}{2}\left(q^{2}-q\right) \sum_{x \in \mathbb{F}_{q} *} \alpha\left(x^{2}\right) \\
= & \frac{1}{|G|}\left[\frac{1}{2}\left(q^{2}+q\right)\left(\sum_{x \in \mathbb{F}_{q}} \alpha\left(x^{2}\right)+\sum_{x, y \in \mathbb{F}_{q}^{*}, x \neq y} \alpha(x) \alpha(y)\right)\right. \\
- & \frac{1}{2}\left(q^{2}-q\right)\left(\sum_{x \in \mathbb{F}_{q} *} \alpha\left(x^{2}\right)+\sum_{x, y \in \mathbb{F}_{q}, y \neq 0} \alpha\left(x^{2}-\epsilon y^{2}\right)\right) \\
& +\frac{2 q^{3}-q^{2}-q+q^{2}-q}{2} \alpha\left(x_{x \in \mathbb{F}_{q} *}\right],
\end{aligned}
$$

which equals

$$
\begin{aligned}
& \frac{1}{\left.q(q-1)^{2}(q+1)\right)}\left[\frac{1}{2}\left(q^{2}+q\right)\left(\sum_{x \in \mathbb{F}_{q}^{*}} \alpha(x)\right)\left(\sum_{y \in \mathbb{F}_{q}^{*}} \alpha(y)\right)-\frac{1}{2}\left(q^{2}-q\right) \sum_{w \in \mathbb{F}_{q^{2}}^{*}} \alpha(\nu(w))\right. \\
& \left.+q\left(q^{2}-1\right) \sum_{x \in \mathbb{F}_{q}^{*}} \alpha^{2}(x)\right] .
\end{aligned}
$$

If $\alpha$ is trivial, then $\alpha^{2}$ is trivial. Then $\mathbb{S}$ reduces to

$$
\left.\frac{1}{\left.q(q-1)^{2}(q+1)\right)}\left[\frac{1}{2}\left(q^{2}+q\right)(q-1)(q-1)-\frac{1}{2}\left(q^{2}-q\right)\right)\left(q^{2}-1\right)+q\left(q^{2}-1\right)(q-1)\right]=1 .
$$


When $\alpha$ is non-trivial, we have two sub-cases.

(i) When $\alpha^{2}$ is non-trivial, we have $\mathbb{S}=0$.

(ii) When $\alpha^{2}$ is trivial then $\alpha(x)=\overline{\alpha(x)}$ for all $x \in \mathbb{F}_{q}^{*}$, which implies $\alpha(r)=-1$. This case divides into two sub-cases depending on the parity of $q$.

○ Assume $q$ to be even. Since $q-1$ is odd, $(-1)^{q-1} \neq 1$. Hence this case cannot occur.

○ If $q$ is odd, then we get $\mathbb{S}=1$.

\subsection{3}

Next, we will find copies of $W_{\alpha, \beta}$ in $V \otimes V$, where $\alpha, \beta$ are distinct characters of $\mathbb{F}_{q}^{*}$.

$$
\begin{aligned}
& \mathbb{S}=\left\langle\chi_{V \otimes V}, \chi_{W_{\alpha, \beta}}\right\rangle \\
= & \frac{1}{|G|}\left[\sum_{x \in \mathbb{F}_{q}^{*}} q^{2}(q+1) \alpha(x) \beta(x) .1+0+\frac{1}{2} \sum_{x, y \in \mathbb{F}_{q}^{*}, x \neq y}(\alpha(x) \beta(y)+\beta(x) \alpha(y))\left(q^{2}+q\right)+0\right] \\
= & \frac{1}{q(q-1)^{2}(q+1)}\left[\sum_{x \in \mathbb{F}_{q}^{*}} q^{2}(q+1) \alpha(x) \beta(x)+\frac{1}{2}\left(q^{2}+q\right)\left(\sum_{x, y \in \mathbb{F}_{q}^{*}, x \neq y} \alpha(x) \beta(y)+\sum_{x \in \mathbb{F}_{q}^{*}} \alpha(x) \beta(x)\right)\right. \\
& \left.+\frac{1}{2}\left(q^{2}+q\right)\left(\sum_{x, y \in \mathbb{F}_{q}^{*}, x \neq y} \beta(x) \alpha(y)+\sum_{x \in \mathbb{F}_{q}^{*}} \alpha(x) \beta(x)\right)-\left(q^{2}+q\right) \sum_{x \in \mathbb{F}_{q}^{*}} \alpha(x) \beta(x)\right] \\
= & \frac{1}{q(q-1)^{2}(q+1)}\left[\frac{1}{2}\left(q^{2}+q\right)\left(\sum_{x \in \mathbb{F}_{q}^{*}} \alpha(x)\right)\left(\sum_{y \in \mathbb{F}_{q}^{*}} \beta(y)\right)+\frac{1}{2}\left(q^{2}+q\right)\left(\sum_{x \in \mathbb{F}_{q}^{*}} \alpha(x)\right)\left(\sum_{y \in \mathbb{F}_{q}^{*}} \beta(y)\right)\right. \\
& \left.+q\left(q^{2}-1\right) \sum_{x \in \mathbb{F}_{q}^{*}} \alpha(x) \beta(x)\right] \\
= & \frac{1}{(q-1)^{2}}\left[\left(\sum_{x \in \mathbb{F}_{q}^{*}} \alpha(x)\right)\left(\sum_{y \in \mathbb{F}_{q}^{*}} \beta(y)\right)+(q-1) \sum_{x \in \mathbb{F}_{q}^{*}} \alpha(x) \beta(x)\right] .
\end{aligned}
$$


The sum is zero if either $\alpha$ or $\beta$ is non-trivial, and $\alpha \beta$ is non-trivial. Since $\alpha, \beta$ cannot both be trivial (since they are unequal), we assume that $\alpha$ and $\beta$ are both non-trivial but $\alpha \beta$ is trivial. Then

$$
\mathbb{S}=\frac{1}{(q-1)^{2}}[0+(q-1)(q-1)]=1
$$

Now, consider the relation $\alpha(x) \cdot \beta(x)=1$ for all $x$, which is true if and only if $\beta(x)=\overline{\alpha(x)}$ for all $x$. In particular, $\beta$ is determined by $\alpha$. As before, this subdivides into cases according to the parity of $q$.

- If $q$ is even, then $q-1$ is odd. Since $\alpha$ is non-trivial, there are $q-2$ possibilities for $\alpha$. Since $W_{\alpha, \beta} \cong W_{\beta, \alpha}$, the number of distinct irreducible representations of the form $W_{\alpha, \beta}$ is $\frac{q-2}{2}$.

- If $q$ is odd, then $q-1$ is even. We cannot have $\alpha(r)= \pm 1$, since that would lead to $\alpha=\beta$. Hence there are $q-3$ possibilities for $\alpha(r)$, and $\frac{q-3}{2}$ possibilities for $W_{\alpha, \beta}$.

\subsection{4}

Finally, we will detect copies of $X_{\phi}$ in $V \otimes V$, where we may assume $\phi \neq \phi^{q}$. In particular, $\phi$ is a non-trivial character. Moreover, $\phi^{q}$ is also non-trivial, since $q$ does not divide $q^{2}-1$. We will use Lemmas 4.5 and 4.6 to simplify the expressions below. We have

$$
\begin{aligned}
& \mathbb{S}=\left\langle\chi_{V \otimes V}, \chi_{X_{\phi}}\right\rangle \\
= & \frac{1}{|G|}\left[\sum_{x \in \mathbb{F}_{q}^{*}} q^{2}(q-1) \phi(x) \cdot 1+0+0-\frac{1}{2} \sum_{x, y \in \mathbb{F}_{q}^{*}, y \neq 0}\left(\phi(x+y \sqrt{\epsilon})+\phi\left((x+y \sqrt{\epsilon})^{q}\right)\left(q^{2}-q\right)\right]\right. \\
= & \frac{q(q-1)}{q(q-1)^{2}(q+1)}\left[q \sum_{x \in \mathbb{F}_{q}^{*}} \phi(x)-\frac{1}{2} \sum_{w \in \mathbb{F}_{q^{2}}^{*} \backslash \mathbb{F}_{q}^{*}}(\phi(w))-\frac{1}{2} \sum_{w \in \mathbb{F}_{q^{2}}^{*} \mathbb{F}_{q}^{*}}\left(\phi^{q}(w)\right)\right] \\
= & \frac{1}{q^{2}-1}\left[q \sum_{x \in \mathbb{F}_{q}^{*}} \phi(x)-\frac{1}{2} \sum_{w \in \mathbb{F}_{q^{2}}^{*}}(\phi(w))+\frac{1}{2} \sum_{x \in \mathbb{F}_{q}^{*}} \phi(x)-\frac{1}{2} \sum_{w \in \mathbb{F}_{q^{2}}^{*}}\left(\phi^{q}(w)\right)+\frac{1}{2} \sum_{x \in \mathbb{F}_{q}^{*}} \phi^{q}(x)\right] .
\end{aligned}
$$


Now $\sum_{w \in \mathbb{F}_{q^{*}}^{*}}(\phi(w))=\sum_{w \in \mathbb{F}_{q^{2}}^{*}}\left(\phi^{q}(w)\right)=0$. For $\mathbb{S}$ to be non-zero, the restriction $\hat{\phi}=\left.\phi\right|_{\mathbb{F}_{q}^{*}}$ must be trivial. In that case,

$$
\mathbb{S}=\frac{1}{q^{2}-1}\left[q(q-1)-0+\frac{1}{2}(q-1)-0+\frac{1}{2}(q-1)\right]=\frac{(q-1)(q+1)}{q^{2}-1}=1
$$

Let $s$ be as in Section 5.1.1, and let $\delta=\phi(s)$. By assumption, $\delta^{q+1}=\phi\left(s^{q+1}\right)=1$, and hence $\delta$ must be a $(q+1)$-th root of unity except 1 .

- Assume $q$ to be even, so that $q+1$ is odd. Then there are $q$ possibilities for $\delta$. Since $X_{\phi} \cong X_{\phi^{q}}$, this gives $\frac{q}{2}$ copies of $X_{\phi}$.

- Assume $q$ to be odd, so that $q+1$ is even. Excluding the case $\delta=-1$ (since otherwise $\left.\phi=\phi^{q}\right)$, there are $q-1$ possibilities for $\delta$ and hence $\frac{q-1}{2}$ copies of $X_{\phi}$.

We summarize these calculations in the following proposition.

Proposition 5.4. If $q$ is odd, then we have a decomposition

$$
V \otimes V \cong U_{\mathbb{T}} \oplus V \oplus V_{\alpha_{0}} \oplus\left(\bigoplus_{\alpha \in Q_{1}} W_{\alpha, \bar{\alpha}}\right) \oplus\left(\bigoplus_{\phi \in Q_{2}} X_{\phi}\right)
$$

where we use the following conventions:

- $\alpha_{0}: \mathbb{F}_{q}^{*} \longrightarrow \mathbb{C}^{*}$ is the unique character of order two.

- $Q_{1}$ is the set of characters $\alpha: \mathbb{F}_{q}^{*} \longrightarrow \mathbb{C}^{*}$ such that $\alpha(r) \neq \pm 1$. We make the convention that $W_{\alpha, \bar{\alpha}}$ and $W_{\bar{\alpha}, \alpha}$ represent the same summand.

- $Q_{2}$ is the set of non-trivial characters $\phi: \mathbb{F}_{q^{2}}^{*} \longrightarrow \mathbb{C}^{*}$ such that $\hat{\phi}$ is trivial and ord $(\phi) \neq 2$. We make the convention that $X_{\phi}$ and $X_{\phi^{q}}$ represent the same summand. 
If $q$ is even, then we have a decomposition

$$
V \otimes V \cong U_{\mathbb{T}} \oplus V \oplus\left(\bigoplus_{\alpha \in Q_{1}} W_{\alpha, \bar{\alpha}}\right) \oplus\left(\bigoplus_{\phi \in Q_{2}} X_{\phi}\right)
$$

where $Q_{1}, Q_{2}$ have the same meaning as above with the following provisos: in the definition of $Q_{1}$, the restriction $\alpha(r) \neq-1$ is unnecessary, and in the definition of $Q_{2}$ the restriction ord $(\phi) \neq 2$ is unnecessary.

We will tally that the dimensions of the two sides in equation (5.1) coincide. The dimension of the left-hand side is $q^{2}$, whereas that of the right-hand side is also

$$
1+q+q+\frac{q-3}{2}(q+1)+\frac{q-1}{2}(q-1)=q^{2}
$$

Similarly, the right-hand side of 5.2 has dimension $1+q+\frac{q-2}{2}(q+1)+\frac{q}{2}(q-1)=q^{2}$. We have performed similar dimension checks for all the decompositions below. Since these calculations are very similar to the present case, the details are omitted.

Example 5.5. We will make this decomposition explicit for $q=5$. We take $\epsilon=2$ (which is a non-square in $\mathbb{F}_{5}$ ), and make the identification

$$
\mathbb{F}_{25}=\frac{\mathbb{F}_{5}[t]}{\left(t^{2}-2\right)}
$$

It is easy to check that $s=t+3$ is a cyclic generator of $\mathbb{F}_{25}^{*}$, and then $r=(t+3)^{6}=2$ is a cyclic generator of $\mathbb{F}_{5}^{*}$.

Each character $\alpha: \mathbb{F}_{5}^{*} \longrightarrow \mathbb{C}^{*}$ is completely specified by $\alpha(r) \in\{1,-1, i,-i\}$. The unique non-trivial order 2 character is the one such that $\alpha_{0}(r)=-1$. Moreover, $Q_{1}$ consists of the two characters

$$
\alpha_{1}(r)=i, \quad \alpha_{2}(r)=-i
$$


which are inverses of each other. Each non-trivial character $\mathbb{F}_{25}^{*} \longrightarrow \mathbb{C}^{*}$ is of the form

$$
\phi_{k}(s)=\exp \left(\frac{2 \pi i k}{24}\right)
$$

for some $k$ such that $1 \leqslant k \leqslant 23$. Then

$$
\phi_{k}(r)=\phi_{k}\left(s^{6}\right)=\exp \left(\frac{\pi i k}{2}\right)
$$

Then $\hat{\phi}_{k}=\left.\phi_{k}\right|_{\mathbb{F}_{5}^{*}}$ is trivial exactly when 4 divides $k$, i.e., $k=4,8,12,16$ or 20 . $\operatorname{But}$ ord $\left(\phi_{k}\right) \neq 2$ implies that $k \neq 12$. Moreover, $\phi_{4}^{5}=\phi_{20}$ and $\phi_{8}^{5}=\phi_{16}$. This gives the following decomposition:

$$
V \otimes V \cong U_{\mathbb{T}} \oplus V \oplus V_{\alpha_{0}} \oplus W_{\alpha_{1}, \alpha_{2}} \oplus X_{\phi_{4}} \oplus X_{\phi_{8}}
$$

Remark 5.6. If $\alpha, \beta$ are arbitrary characters $\mathbb{F}_{q}^{*} \longrightarrow \mathbb{C}$, then

$$
V_{\alpha} \otimes V_{\beta} \cong\left(V \otimes U_{\alpha}\right) \otimes\left(V \otimes U_{\beta}\right) \cong(V \otimes V) \otimes U_{\alpha \beta},
$$

which can be decomposed into irreducibles using the proposition above.

\subsection{The Decomposition of $V \otimes W_{\alpha, 1}$}

Now we will find the irreducible decomposition of $V \otimes W_{\alpha, 1}$ using the same technique. The character table is as follows:

\begin{tabular}{|c||c|c|c|c|}
\hline & $a_{x}$ & $b_{x}$ & $c_{x, y}$ & $d_{x, y}$ \\
\hline$\chi_{V \otimes W_{\alpha, 1}}$ & $q(q+1) \alpha(x)$ & 0 & $\alpha(x)+\alpha(y)$ & 0 \\
\hline
\end{tabular}




\section{4 .1}

By Lemma 5.3, there are no copies of $U_{\beta}$ in $V \otimes W_{\alpha, 1}$.

Let $\beta$ be an arbitrary character of $\mathbb{F}_{q}^{*}$. By Lemma 5.2, we have equalities

$$
m\left(V_{\beta}, V \otimes W_{\alpha, 1}\right)=m\left(W_{\alpha^{-1}, 1}, V \otimes V_{\beta^{-1}}\right)=m\left(W_{\alpha^{-1} \beta, \beta}, V \otimes V\right) .
$$

The last number is given by Proposition 5.4. We deduce that for each character $\beta$ of $\mathbb{F}_{q}^{*}$ such that $\alpha^{-1} \beta=\beta^{-1}$ (i.e., $\beta^{2}=\alpha$ ), we get one copy of $V_{\beta}$ in the decomposition.

\section{4 .2}

Now we will find copies of $W_{\beta, \gamma}$, where $\beta, \gamma$ are distinct characters of $\mathbb{F}_{q}^{*}$. We have

$$
\begin{aligned}
\mathbb{S}=\left\langle\chi_{V \otimes W_{\alpha, 1}}, \chi_{W_{\beta, \gamma}}\right\rangle \\
=\frac{1}{|G|}\left[\sum_{a_{x}}\left(q(q+1)^{2} \alpha \overline{\beta \gamma}(x)+\left(\frac{q^{2}+q}{2}\right) \sum_{c_{x, y}}(\alpha(x)+\alpha(y)) \overline{(\beta(x) \gamma(y)+\beta(y) \gamma(x))}\right]\right. \\
=\frac{1}{q(q-1)^{2}(q+1)}\left[\sum_{x \in \mathbb{F}_{q}^{*}} q(q+1)^{2} \alpha \cdot \overline{\beta \cdot \gamma}(x)+\frac{q^{2}+q}{2}\left(\sum_{x, y \in \mathbb{F}_{q}^{*}, x \neq y} \alpha(x) \overline{\beta(x) \gamma(y)}\right.\right. \\
\left.\left.+\sum_{x, y \in \mathbb{F}_{q}^{*}, x \neq y} \alpha(x) \overline{\beta(y) \gamma(x)}+\sum_{x, y \in \mathbb{F}_{q}^{*}, x \neq y} \alpha(y) \overline{\beta(x) \gamma(y)}+\sum_{x, y \in \mathbb{F}_{q}^{*}, x \neq y} \alpha(y) \overline{\beta(y) \gamma(x)}\right)\right] \\
=\frac{1}{q(q-1)^{2}(q+1)}\left[\left(q(q+1)^{2}-2\left(q^{2}+q\right)\right) \sum_{x \in \mathbb{F}_{q}^{*}} \alpha \cdot \overline{\beta \cdot \gamma}(x)\right. \\
+\left(q^{2}+q\right)\left(\sum_{x \in \mathbb{F}_{q}^{*}} \alpha \cdot \bar{\beta}(x)\right)\left(\sum_{y \in \mathbb{F}_{q}^{*}} \bar{\gamma}(y)\right)+\left(q^{2}+q\right)\left(\sum_{y \in \mathbb{F}_{q}^{*}} \alpha \cdot \bar{\gamma}(y)\right)(\underbrace{\left.\left.\sum_{x \in \mathbb{F}_{q}^{*}} \bar{\beta}(x)\right)\right]}_{(1)} \\
=\frac{1}{(q-1)^{2}}[(q-1) \underbrace{\alpha \cdot \bar{\beta} \cdot \gamma(x)}_{\left(2 \in \mathbb{F}_{q}^{*}\right.}+(\underbrace{\left.\left(\sum_{y \in \mathbb{F}_{q}^{*}} \alpha \cdot \bar{\gamma}(y)\right)\left(\sum_{x \in \mathbb{F}_{q}^{*}} \bar{\beta}(x)\right)\right]}_{\left(\sum_{x \in \mathbb{F}_{q}^{*}} \alpha \cdot \bar{\beta}(x)\right)\left(\sum_{y \in \mathbb{F}_{q}^{*}} \bar{\gamma}(y)\right)} .
\end{aligned}
$$

For $\mathbb{S}$ to be non-zero, at least one of the terms from (1), (2), (3) must be non-zero. 
- The term (2) is non-zero exactly when $\gamma$ is trivial and $\alpha=\beta$.

- The term (3) is non-zero exactly when $\beta$ is trivial and $\alpha=\gamma$.

- The term (1) is non-zero exactly when $\alpha=\beta \gamma$.

Since $\beta \neq \gamma$, either (2) or (3) must be zero. We have the following cases:

- If (2) is non-zero, then (1) is also non-zero and then $\mathbb{S}=2$.

- If (3) is non-zero, then (1) is also non-zero and then $\mathbb{S}=2$. But this is the same as the previous case, since $W_{\beta, \gamma} \cong W_{\gamma, \beta}$.

- Assume (2), (3) are both zero, but (1) is non-zero. This case is further divided into two sub-cases:

- Assume that $\alpha$ is not a square, so that $q$ is necessarily odd. Then there are $\frac{(q-3)}{2}$ possibilities for the unordered pair $\{\beta, \gamma\}=\left\{\beta, \alpha \beta^{-1}\right\}$. For each such pair, there is one copy of $W_{\beta, \gamma}$ in the tensor product.

○ Assume that $\alpha$ is a square. Then we must exclude those $\beta$ such that $\beta=\alpha \beta^{-1}$. Hence, if $q$ is even, there are $\frac{q-4}{2}$ possibilities for the pair $\left\{\beta, \alpha \beta^{-1}\right\}$. When $q$ is odd, there are $\frac{q-5}{2}$ possibilities. For each such pair, there is one copy of $W_{\beta, \gamma}$ in the tensor product.

This gives a precise description of the summands $W_{\beta, \gamma}$ in $V \otimes W_{\alpha, 1}$. 


\section{4 .3}

Finally, we find copies of $X_{\phi}$, where $\phi: \mathbb{F}_{q^{2}}^{*} \longrightarrow \mathbb{C}$ is a character such that $\phi \neq \phi^{q}$. We have

$$
\begin{aligned}
\mathbb{S} & =\left\langle\chi_{V \otimes W_{\alpha, 1}}, \chi_{X_{\phi}}\right\rangle=\frac{1}{|G|}\left[\sum_{a_{x}}(q(q+1)(q-1) \alpha(x) \overline{\phi(x)}]\right. \\
& =\frac{1}{q(q-1)^{2}(q+1)}\left[q(q+1)(q-1) \sum_{x \in \mathbb{F}_{q}^{*}} \alpha(x) \overline{\phi(x)}\right] .
\end{aligned}
$$

Then $\mathbb{S}=1$ when $\hat{\phi}=\left.\phi\right|_{\mathbb{F}_{q}^{*}}=\alpha$ and $\mathbb{S}=0$ otherwise. Thus, for each character $\phi$ satisfying the condition $\hat{\phi}=\alpha$, we have one copy of $X_{\phi}$ in $V \otimes W_{\alpha, 1}$.

These calculations are summarized in the following proposition.

Proposition 5.7. When $\alpha$ is a non-square, we have a decomposition

$$
V \otimes W_{\alpha, 1} \cong\left(W_{\alpha, 1}\right)^{\oplus 2} \oplus\left(\bigoplus_{\beta \in Q_{1}} W_{\beta, \alpha \beta^{-1}}\right) \oplus\left(\bigoplus_{\phi \in Q_{2}} X_{\phi}\right)
$$

where we use the following conventions:

- $Q_{1}$ is the set of characters $\{\beta: \beta \neq \alpha, 1\}$ of $\mathbb{F}_{q}^{*}$. Moreover, it is understood that $W_{\beta, \alpha \beta^{-1}}$ and $W_{\alpha \beta^{-1, \beta}}$ represent the same summand.

- $Q_{2}$ is the set of characters $\phi$ of $\mathbb{F}_{q^{2}}^{*}$ such that $\phi \neq \phi^{q}$ and $\left.\phi\right|_{\mathbb{F}_{q}^{*}}=\alpha$. Moreover, it is understood that $X_{\phi}$ and $X_{\phi^{q}}$ represent the same summand.

When $\alpha$ is a square, we have a decomposition

$$
V \otimes W_{\alpha, 1} \cong\left(W_{\alpha, 1}\right)^{\oplus 2} \oplus\left(\bigoplus_{\beta \in Q_{1}} W_{\beta, \alpha \beta^{-1}}\right) \oplus\left(\bigoplus_{\phi \in Q_{2}} X_{\phi}\right) \oplus\left(\bigoplus_{\beta^{2}=\alpha} V_{\beta}\right)
$$

where $Q_{1}, Q_{2}$ have the same meaning as before. 
As mentioned earlier, we have carried out a dimension check as in the previous section but omitted the details.

Remark 5.8. If $\alpha, \beta, \gamma: \mathbb{F}_{q}^{*} \longrightarrow \mathbb{C}$ are arbitrary characters (such that $\beta \neq \gamma$ ), then

$$
V_{\alpha} \otimes W_{\beta, \gamma} \cong\left(V \otimes U_{\alpha}\right) \otimes\left(W_{\beta \gamma^{-1}, 1} \otimes U_{\gamma}\right) \cong\left(V \otimes W_{\beta \gamma^{-1}, 1}\right) \otimes U_{\alpha \gamma}
$$

which can be decomposed using the calculation above.

Example 5.9. We will continue Example 5.5 from the previous section. Let $\alpha_{0}$ be the non-trivial character of order 2 , and consider $V \otimes W_{\alpha_{0}, 1}$. Then $\beta=\alpha_{1}, \alpha_{2}$ are the characters such that $\beta^{2}=\alpha$. However, the set $Q_{1}$ is empty.

The condition $\hat{\phi}_{k}=\alpha_{0}$ implies that $\exp \left(\frac{\pi i k}{2}\right)=-1$, i.e., $k=2,6,10,14,18$ or 22 . But $\phi^{5} \neq \phi$ implies that $k \neq 6,18$. Since $X_{\phi_{2}}=X_{\phi_{10}}$ and $X_{\phi_{14}}=X_{\phi_{22}}$, we get the decomposition

$$
V \otimes W_{\alpha_{0}, 1} \cong W_{\alpha_{0}, 1}^{\oplus 2} \oplus X_{\phi_{2}} \oplus X_{\phi_{14}} \oplus V_{\alpha_{1}} \oplus V_{\alpha_{2}}
$$

It is a general feature of our calculations that, given specific irreducible representations $E_{1}$ and $E_{2}$, finding the summands in $E_{1} \otimes E_{2}$ reduces to solving a simple set of congruence equations modulo $q-1$ or $q^{2}-1$

Henceforth we will increasingly make use of Lemma 5.2 to reduce the number of computations. We will confine ourselves to identifying the representations of each type in the decomposition instead of stating the corresponding propositions explicitly.

\subsection{The Decomposition of $V \otimes X_{\phi}$}

Now we consider the decomposition of $V \otimes X_{\phi}$, where $\phi$ is a character of $\mathbb{F}_{q^{2}}^{*}$. The character table is as follows: 


\begin{tabular}{|c||c|c|c|c|}
\hline & $a_{x}$ & $b_{x}$ & $c_{x, y}$ & $d_{x, y}$ \\
\hline \hline$\chi_{V \otimes X_{\phi}}$ & $q(q-1) \phi(x)$ & 0 & 0 & $\left(\phi(w)+\phi\left(w^{q}\right)\right)$ \\
\hline
\end{tabular}

Using Lemmas 5.2 and 5.3 , we deduce the following statements:

- There are no copies of $U_{\alpha}$ in $V \otimes X_{\phi}$.

- We have

$$
m\left(V_{\alpha}, V \otimes X_{\phi}\right)=m\left(X_{\phi^{-1}} \otimes U_{\alpha}, V \otimes V\right)=m\left(X_{\phi^{-1} \widetilde{\alpha}}, V \otimes V\right) .
$$

The last number is known from the calculations in Section 5.3. By Proposition 5.4, the condition $\phi^{-1} \widetilde{\alpha} \in Q_{2}$ implies that $\left.\left(\phi^{-1} \widetilde{\alpha}\right)\right|_{\mathbb{F}_{q}^{*}}=1$. Thus we get $\phi^{-1}(x) \widetilde{\alpha}(x)=1$ for all $x \in \mathbb{F}_{q}^{*}$, i.e., $\hat{\phi}=\alpha^{2}$. Hence we get one copy of $V_{\alpha}$ when $\hat{\phi}=\alpha^{2}$ and $\operatorname{ord}\left(\phi^{-1} \widetilde{\alpha}\right) \neq 2$.

- We have

$$
m\left(W_{\alpha, \beta}, V \otimes X_{\phi}\right)=m\left(X_{\phi^{-1}}, V \otimes W_{\alpha^{-1}, \beta^{-1}}\right)=m\left(X_{\phi^{-1} \tilde{\beta}}, V \otimes W_{\alpha^{-1} \beta, 1}\right) .
$$

The last number is known from the calculations in Section 5.4. By Proposition 5.7, the condition $\phi^{-1} \widetilde{\beta} \in Q_{2}$ implies that $\left.\left(\phi^{-1} \widetilde{\beta}\right)\right|_{\mathbb{F}_{q}^{*}}=\alpha^{-1} \beta$. Thus we get $\phi^{-1}(x) \widetilde{\beta}(x)=\alpha^{-1}(x) \beta(x)$ for all $x \in \mathbb{F}_{q}^{*}$, i.e., $\hat{\phi}^{-1} \beta^{2}=\alpha^{-1} \beta$ for all $x \in \mathbb{F}_{q}^{*}$. Hence there will be one copy of $W_{\alpha, \beta}$ exactly when $\hat{\phi}=\alpha \beta$. 


\subsection{1}

Now we detect copies of $X_{\psi}$, where $\psi$ is a character of $\mathbb{F}_{q^{2}}^{*}$. As usual, assume $\psi \neq \psi^{q}$. Recall that $\hat{\phi}=\left.\phi\right|_{\mathbb{F}_{q}^{*}}$ and $\hat{\psi}=\left.\psi\right|_{\mathbb{F}_{q}^{*}}$. Then

$$
\begin{aligned}
& \mathbb{S}=\left\langle\chi_{V \otimes X_{\phi}}, \chi_{X_{\psi}}\right\rangle \\
= & \left.\frac{1}{|G|}\left[\sum_{x \in \mathbb{F}_{q}^{*}} q(q-1) \phi(x)\right)(\overline{(q-1) \psi(x)})-\left(\frac{q(q-1)}{2}\right) \cdot \sum_{w \in \mathbb{F}_{q^{*}} \mathbb{F}_{q}^{*}}\left(\phi(w)+\phi\left(w^{q}\right)\right)\left(\overline{\psi(w)+\psi\left(w^{q}\right)}\right)\right] \\
= & \frac{1}{q(q-1)^{2}(q+1)}\left[(q-1)^{2} q \sum_{x \in \mathbb{F}_{q}^{*}} \phi \cdot \bar{\psi}(x)\right. \\
- & \left(\frac{q(q-1)}{2}\right) \sum_{w \in \mathbb{F}_{q^{2}}}\left(\phi \mathbb{F}_{q}^{*}\right. \\
= & \frac{1}{q(q-1)^{2}(q+1)}\left[(q-1)^{2} q \sum_{x \in \mathbb{F}_{q}^{*}} \phi \cdot \bar{\psi}(x)-\left(\frac{q(q-1)}{2}\right) \cdot\left(\sum_{w \in \mathbb{F}_{q^{2}}} \phi \bar{\psi}(w)+\sum_{w \in \mathbb{F}_{q^{2}}} \phi(w) \overline{\psi\left(w^{q}\right)}+\phi\left(w^{q}\right) \overline{\psi(w)}+\phi \cdot \bar{\psi}\left(w^{q}\right)\right)\right] \\
+ & \left.\sum_{w \in \mathbb{F}_{q^{2}}} \phi^{q}(w) \overline{\psi(w)}+\sum_{w \in \mathbb{F}_{q^{2}}}(\phi \bar{\psi})^{q}(w)\right)+\frac{q(q-1)}{2} \sum_{x \in \mathbb{F}_{q}^{*}} \phi^{q}(x) \overline{\psi(x)}+\frac{q(q-1)}{2} \sum_{x \in \mathbb{F}_{q}^{*}} \phi(x) \overline{(\psi)^{q}(x)} \\
& \left.+q(q-1) \sum_{x \in \mathbb{F}_{q}^{*}} \phi \bar{\psi}(x)\right] \cdot
\end{aligned}
$$

Since $\hat{\phi}^{q}=\hat{\phi}$ and $\hat{\psi}^{q}=\hat{\psi}$, this simplifies to

$$
\frac{q+1}{q^{2}-1} \underbrace{\sum_{x \in \mathbb{F}_{q}^{*}} \phi \bar{\psi}(x)}_{S_{0}}-\frac{1}{2\left(q^{2}-1\right)}[\underbrace{\sum_{w \in \mathbb{F}_{q^{2}}} \phi \bar{\psi}(w)}_{S_{1}}+\underbrace{\sum_{w \in \mathbb{F}_{q^{*}}} \phi \bar{\psi}^{q}(w)}_{S_{2}}+\underbrace{\sum_{w \in \mathbb{F}_{q^{*}}} \phi^{q} \bar{\psi}(w)}_{S_{3}}+\underbrace{\sum_{w \in \mathbb{F}_{q^{*}}} \phi^{q} \bar{\psi}^{q}(w)}_{S_{4}}] .
$$

We have five sums labelled $S_{0}, \ldots S_{4}$. Now consider the following five conditions on $\phi$ and $\psi$.

$$
C 0 . \hat{\phi}=\hat{\psi}, \quad C 1 . \phi=\psi, \quad C 2 . \phi=\psi^{q}, \quad C 3 . \phi^{q}=\psi, \quad C 4 . \phi^{q}=\psi^{q} .
$$

By Lemma 4.5 we know that $S_{0}=q-1$ if condition $C 0$ is satisfied, and $S_{0}=0$ otherwise. For 
$1 \leqslant i \leqslant 4$, by Lemma 4.6 we know that $S_{i}=q^{2}-1$ if $C i$ is satisfied, and $S_{i}=0$ otherwise. Moreover, $C 1 \Longleftrightarrow C 4$ and $C 2 \Longleftrightarrow C 3$. (This follows because $\phi^{q^{2}}=\phi$ and $\psi^{q^{2}}=\psi$.) If any of the conditions $C 1, \ldots, C 4$ holds, then $C 0$ also holds. Moreover, at most one of the conditions from $C 1, C 2$ can hold, since otherwise $\psi=\psi^{q}$.

Thus we have the following possibilities:

- If none of the conditions $C 0, \ldots, C 4$ holds, then $\mathbb{S}=0$.

- If any of the conditions $C 1, \ldots, C 4$ holds, then two of them hold and $C 0$ also holds. Then

$$
\mathbb{S}=\frac{q+1}{q^{2}-1}(q-1)-\frac{2\left(q^{2}-1\right)}{2\left(q^{2}-1\right)}=0
$$

- If only $C 0$ holds and none of $C 1, \ldots, C 4$ holds, then $\mathbb{S}=\frac{q+1}{q^{2}-1}(q-1)=1$.

Hence $X_{\psi}$ appears in the decomposition of $V \otimes X_{\phi}$, if and only if $\hat{\phi}=\hat{\psi}$ but $\psi \neq \phi, \phi^{q}$. Moreover, then it appears with multiplicity one. This completes the calculation for the case $V \otimes X_{\phi}$.

We summarize the result in the following proposition.

Proposition 5.10. When $\hat{\phi}$ is a non-square, we have a decomposition

$$
V \otimes X_{\phi} \cong\left(\bigoplus_{\alpha} W_{\alpha, \hat{\phi} \alpha^{-1}}\right) \oplus\left(\bigoplus_{\psi \in Q} X_{\psi}\right)
$$

where $Q$ is the set of characters $\psi$ of $\mathbb{F}_{q^{2}}^{*}$ such that $\hat{\psi}=\hat{\phi}$ but $\psi \neq \phi, \phi^{q}$. Moreover, it is understood that $X_{\psi}$ and $X_{\psi^{q}}$ stand for the same summand.

When $\hat{\phi}$ is a square, we have a decomposition

$$
V \otimes X_{\phi} \cong\left(\bigoplus_{\alpha} W_{\alpha, \hat{\phi} \alpha^{-1}}\right) \oplus\left(\bigoplus_{\psi \in Q} X_{\psi}\right) \oplus\left(\bigoplus_{\eta} V_{\eta}\right)
$$


where $Q$ has the same meaning as above, and in the first summand those $\alpha$ for which $\alpha^{2}=\hat{\phi}$ are disallowed. The last sum is over characters $\eta$ of $\mathbb{F}_{q}^{*}$ such that $\eta^{2}=\hat{\phi}$.

Remark 5.11. For any character $\alpha$ of $\mathbb{F}_{q}^{*}$, we have an isomorphism

$$
V_{\alpha} \otimes X_{\phi} \cong\left(V \otimes X_{\phi}\right) \otimes U_{\alpha}
$$

which can decomposed using the calculation above.

\subsection{The Decomposition of $W_{\alpha, 1} \otimes X_{\phi}$}

Let $\alpha: \mathbb{F}_{q}^{*} \longrightarrow \mathbb{C}^{*}$ and $\phi: \mathbb{F}_{q^{2}}^{*} \longrightarrow \mathbb{C}^{*}$ be characters. We proceed to decompose the tensor product $W_{\alpha, 1} \otimes X_{\phi}$. As usual, assume $\phi \neq \phi^{q}$. The character table is as follows:

\begin{tabular}{|c||c|c|c|c|}
\hline & $a_{x}$ & $b_{x}$ & $c_{x, y}$ & $d_{x, y}$ \\
\hline \hline$\chi_{W_{\alpha, 1} \otimes X_{\phi}}$ & $\left(q^{2}-1\right) \alpha(x) \phi(x)$ & $-\alpha(x) \phi(x)$ & 0 & 0 \\
\hline
\end{tabular}

Using Lemmas 5.2 and 5.3 , we deduce the following statements:

- There are no copies of $U_{\beta}$ in the decomposition.

- We have

$$
m\left(V_{\beta}, W_{\alpha, 1} \otimes X_{\phi}\right)=m\left(X_{\phi^{-1}}, V_{\beta^{-1}} \otimes W_{\alpha, 1}\right)=m\left(X_{\phi^{-1} \widetilde{\beta}}, V \otimes W_{\alpha, 1}\right) .
$$

The last number is known by the calculations in Section 5.4. By Proposition 5.7, the condition $\phi^{-1} \widetilde{\beta} \in Q_{2}$ implies that $\left.\left(\phi^{-1} \widetilde{\beta}\right)\right|_{\mathbb{F}_{q}^{*}}=\alpha$. Thus we get $\phi^{-1}(x) \widetilde{\beta}(x)=\alpha(x)$ for all $x \in \mathbb{F}_{q}^{*}$. Hence, $\phi^{-1}(x) \beta^{2}(x)=\alpha(x)$ for all $x \in \mathbb{F}_{q}^{*}$. Thus there will be one copy of $V_{\beta}$ when $\alpha \hat{\phi}=\beta^{2}$. 
Now we will find copies of $W_{\beta, \gamma}$, where $\beta$ and $\gamma$ are distinct characters of $\mathbb{F}_{q}^{*}$. We have

$$
\begin{aligned}
& \mathbb{S}=\left\langle\chi_{W_{\alpha, 1} \otimes X_{\phi}}, \chi_{W_{\beta, \gamma}}\right\rangle \\
= & \frac{1}{|G|}\left[\sum_{a_{x}}\left(q^{2}-1\right)(q+1) \alpha(x) \phi(x) \overline{\beta(x) \gamma(x)}-\sum_{b_{x}} \alpha(x) \phi(x) \overline{\beta(x) \gamma(x)}\left(q^{2}-1\right) .\right] \\
= & \frac{q\left(q^{2}-1\right)}{q(q-1)^{2}(q+1)} \sum_{x \in \mathbb{F}_{q}^{*}} \alpha(x) \phi(x) \overline{\beta(x) \gamma(x)} .
\end{aligned}
$$

Hence $\mathbb{S}=1$ when $\beta \gamma=\alpha \hat{\phi}$, and $\mathbb{S}=0$ otherwise.

Finally, we will find copies of $X_{\psi}$, where $\psi$ is a character of $\mathbb{F}_{q^{2}}^{*}$. We have

$$
\begin{aligned}
& \mathbb{S}=\left\langle\chi_{W_{\alpha, 1} \otimes X_{\phi}}, \chi_{X_{\psi}}\right\rangle \\
= & \frac{1}{|G|}\left[\sum_{a_{x}}\left(q^{2}-1\right)(q-1) \alpha(x) \phi(x) \overline{\psi(x)}+\sum_{b_{x}} \alpha(x) \phi(x) \overline{\psi(x)}\left(q^{2}-1\right) .\right] \\
= & \frac{q\left(q^{2}-1\right)}{q(q-1)^{2}(q+1)} \sum_{x \in \mathbb{F}_{q}^{*}} \alpha(x) \phi(x) \overline{\psi(x)} .
\end{aligned}
$$

Hence $\mathbb{S}=1$ when $\hat{\psi}=\alpha \hat{\phi}$, and $\mathbb{S}=0$ otherwise. This completes the calculation.

We summarize the result in the following proposition.

Proposition 5.12. When $\alpha \hat{\phi}$ is a non-square, we have a decomposition

$$
W_{\alpha, 1} \otimes X_{\phi} \cong\left(\bigoplus_{\beta} W_{\beta, \alpha \hat{\phi} \beta^{-1}}\right) \oplus\left(\bigoplus_{\psi \in Q} X_{\psi}\right)
$$

where $Q$ is the set of characters $\psi$ of $\mathbb{F}_{q^{2}}^{*}$ such that $\hat{\psi}=\alpha \hat{\phi}$.

When $\alpha \hat{\phi}$ is a square, then we have a decomposition

$$
W_{\alpha, 1} \otimes X_{\phi} \cong\left(\bigoplus_{\beta} W_{\beta, \alpha \hat{\phi} \beta^{-1}}\right) \oplus\left(\bigoplus_{\psi \in Q} X_{\psi}\right) \oplus\left(\bigoplus_{\eta} V_{\eta}\right)
$$


where $Q$ has the same meaning as before, and in the first summand those $\beta$ such that $\beta^{2}=\alpha \hat{\phi}$ are disallowed. The last summand is over those $\eta$ such that $\eta^{2}=\alpha \hat{\phi}$.

As a consequence, we can decompose any representation of the form

$$
W_{\alpha, \beta} \otimes X_{\phi} \cong\left(W_{\alpha \beta^{-1}, 1} \otimes X_{\phi}\right) \otimes U_{\beta}
$$

\subsection{The Decomposition of $X_{\phi} \otimes X_{\psi}$}

In this section, we will find the irreducible decomposition of $X_{\phi} \otimes X_{\psi}$, where $\phi$ and $\psi$ are characters of $\mathbb{F}_{q^{2}}^{*}$. As usual, assume $\phi \neq \phi^{q}$ and $\psi \neq \psi^{q}$. We have the following character table:

\begin{tabular}{|c||c|c|c|c|}
\hline & $a_{x}$ & $b_{x}$ & $c_{x, y}$ & $d_{x, y}$ \\
\hline \hline$\chi_{X_{\phi} \otimes X_{\psi}}$ & $(q-1)^{2} \phi(x) \psi(x)$ & $\phi(x) \psi(x)$ & 0 & $\left(\phi(w)+\phi\left(w^{q}\right)\right)\left(\psi(w)+\psi\left(w^{q}\right)\right)$ \\
\hline
\end{tabular}

Recall that for $d_{x, y}=\left(\begin{array}{cc}x & \epsilon y \\ y & x\end{array}\right)$, we have the notation $w=x+y t=x+y \sqrt{\epsilon}$.

\section{7 .1}

Using Lemma 5.2 and 5.3 , we deduce the following statements:

- We have

$$
m\left(U_{\alpha}, X_{\phi} \otimes X_{\psi}\right)=m\left(X_{\phi^{-1}}, X_{\psi}\right)
$$

This number can be non-zero only if both $X$-type representations are in fact isomorphic, and in that case it is $=1$. Consider the following four conditions:
(a) $\phi \psi=\tilde{\alpha}$
(b) $\phi \psi^{q}=\tilde{\alpha}$
(c) $\phi^{q} \psi=\tilde{\alpha}$,
(d) $\phi^{q} \psi^{q}=\tilde{\alpha}$. 
Recall that $\tilde{\alpha}^{q}=\tilde{\alpha}$. Hence (a), (d) are equivalent (i.e., they are both simultaneously true or false), and (b), (c) are equivalent. Moreover, if (a), (d) are true then (b), (c) must be false and conversely, since otherwise we would get $\psi^{q}=\psi$. Now $X_{\phi^{-1} \tilde{\alpha}} \cong X_{\psi}$ if and only if, either the pair (a), (d) is true or the pair (b), (c) is true. We have a single copy of $U_{\alpha}$ in each case. By restricting the conditions to $\mathbb{F}_{q}^{*}$, we deduce that $\hat{\phi} \hat{\psi}=\alpha^{2}$.

- As to $V_{\alpha}$, we have

$$
m\left(V_{\alpha}, X_{\phi} \otimes X_{\psi}\right)=m\left(X_{\psi^{-1}}, V_{\alpha^{-1}} \otimes X_{\phi}\right)=m\left(X_{\psi^{-1} \tilde{\alpha}}, V \otimes X_{\phi}\right)
$$

The last number is known by the calculations in Section 5.5. By Proposition 5.10, the condition $\psi^{-1} \widetilde{\alpha} \in Q_{2}$ implies that $\left.\left(\psi^{-1} \widetilde{\alpha}\right)\right|_{\mathbb{F}_{q}^{*}}=\left.\phi\right|_{\mathbb{F}_{q}^{*}}$. We get $\psi^{-1}(x) \widetilde{\alpha}(x)=\phi(x)$ for all $x \in \mathbb{F}_{q}^{*}$, and thus there will be one copy of $V_{\alpha}$ when $\hat{\phi} \hat{\psi}=\alpha^{2}$ and $\psi^{-1} \tilde{\alpha} \neq \phi, \phi^{q}$.

- We have

$$
m\left(W_{\alpha, \beta}, X_{\phi} \otimes X_{\psi}\right)=m\left(X_{\psi^{-1}}, W_{\alpha^{-1}, \beta^{-1}} \otimes X_{\phi}\right)=m\left(X_{\psi^{-1} \tilde{\beta}}, W_{\alpha^{-1} \beta, 1} \otimes X_{\phi}\right)
$$

The last number is known from the calculations in Section 5.6. By Proposition 5.12, the condition $\psi^{-1} \tilde{\beta} \in Q_{2}$ implies that $\left.\left(\psi^{-1} \tilde{\beta}\right)\right|_{\mathbb{F}_{q}^{*}}=\left(\alpha^{-1} \beta\right)$. $\left.\phi\right|_{\mathbb{F}_{q}^{*}}$. Hence we get $\psi^{-1}(x) \widetilde{\beta}(x)=$ $\alpha^{-1}(x) \beta(x) \phi(x)$ for all $x \in \mathbb{F}_{q}^{*}$, i.e., $\psi^{-1}(x) \beta^{2}(x)=\alpha^{-1}(x) \beta(x) \phi(x)$ for all $x \in \mathbb{F}_{q}^{*}$. Thus there will be one copy of $W_{\alpha, \beta}$ when $\hat{\phi} \hat{\psi}=\alpha \beta$. 


\section{7 .2}

Finally, we find copies of $X_{\theta}$, where as usual we assume $\theta^{q} \neq \theta$. We have

$$
\begin{aligned}
& \mathbb{S}=\left\langle\chi_{X_{\phi} \otimes X_{\psi}}, \chi_{X_{\theta}}\right\rangle \\
& =\frac{1}{|G|}\left[\sum_{a_{x}}(q-1)^{3} \phi(x) \psi(x)(\overline{\theta(x)})-\sum_{b_{x}} \phi(x) \psi(x)(\overline{\theta(x)})\left(q^{2}-1\right)\right. \\
& \left.-\left(\frac{q(q-1)}{2}\right) \cdot \sum_{w \in \mathbb{F}_{q^{2}}^{*} \backslash \mathbb{F}_{q}^{*}}\left(\phi(w)+\phi\left(w^{q}\right)\right)\left(\psi(w)+\psi\left(w^{q}\right)\right)\left(\overline{\theta(w)+\theta\left(w^{q}\right)}\right)\right] \\
& =\frac{1}{q(q-1)^{2}(q+1)}\left[q(q-1)(q-3) \sum_{x \in \mathbb{F}_{q}^{*}} \phi \cdot \psi \cdot \bar{\theta}(x)-\left(\frac{q(q-1)}{2}\right) \sum_{w \in \mathbb{F}_{q^{2}} \backslash \mathbb{F}_{q}^{*}}(\phi(w) \psi(w) \overline{\theta(w)}\right. \\
& +\phi(w) \psi\left(w^{q}\right) \overline{\theta(w)}+\phi(w) \psi(w) \overline{\theta\left(w^{q}\right)}+\phi(w) \psi\left(w^{q}\right) \overline{\theta\left(w^{q}\right)}+\phi\left(w^{q}\right) \psi\left(w^{q}\right) \overline{\theta(w)} \\
& \left.\left.+\phi\left(w^{q}\right) \psi(w) \overline{\theta(w)}+\phi\left(w^{q}\right) \psi(w) \overline{\theta\left(w^{q}\right)}+\phi\left(w^{q}\right) \psi\left(w^{q}\right) \overline{\theta\left(w^{q}\right)}\right)\right] \\
& =\frac{1}{q(q-1)^{2}(q+1)}\left[q(q-1)(q-3) \sum_{x \in \mathbb{F}_{q}^{*}} \phi \cdot \psi \cdot \bar{\theta}(x)-\left(\frac{q(q-1)}{2}\right)\left(\left(\sum_{w \in \mathbb{F}_{q^{*}}^{*}} \phi(w) \psi(w) \overline{\theta(w)}\right)\right.\right. \\
& +\left(\sum_{w \in \mathbb{F}_{q^{*}}^{*}} \phi(w) \psi\left(w^{q}\right) \overline{\theta(w)}\right)+\left(\sum_{w \in \mathbb{F}_{q^{*}}^{*}} \phi(w) \psi(w) \overline{\theta\left(w^{q}\right)}\right)+\left(\sum_{w \in \mathbb{F}_{q^{2}}^{*}} \phi(w) \psi\left(w^{q}\right) \overline{\theta\left(w^{q}\right)}\right) \\
& +\left(\sum_{w \in \mathbb{F}_{q^{*}}^{*}} \phi\left(w^{q}\right) \psi\left(w^{q}\right) \overline{\theta(w)}\right)+\left(\sum_{w \in \mathbb{F}_{q^{2}}} \phi\left(w^{q}\right) \psi(w) \overline{\theta(w)}\right)+\left(\sum_{w \in \mathbb{F}_{q^{2}}^{*}} \phi\left(w^{q}\right) \psi(w) \overline{\theta\left(w^{q}\right)}\right) \\
& \left.\left.+\left(\sum_{w \in \mathbb{F}_{q^{2}}^{*}} \phi\left(w^{q}\right) \psi\left(w^{q}\right) \overline{\theta\left(w^{q}\right)}\right)\right)+4 q(q-1) \sum_{x \in \mathbb{F}_{q}^{*}} \phi \cdot \psi \cdot \bar{\theta}(x)\right] .
\end{aligned}
$$


This reduces to the expression

$$
\begin{aligned}
& \frac{1}{q-1} \sum_{x \in \mathbb{F}_{q}^{*}} \phi \cdot \psi \cdot \bar{\theta}(x)-\frac{1}{2\left(q^{2}-1\right)}\left(\sum_{w \in \mathbb{F}_{q^{2}}^{*}} \phi(w) \psi(w) \overline{\theta(w)}\right. \\
& +\sum_{w \in \mathbb{F}_{q^{*}}^{*}} \phi(w) \psi^{q}(w) \overline{\theta(w)}+\sum_{w \in \mathbb{F}_{q^{*}}^{*}} \phi(w) \psi(w) \overline{\theta^{q}(w)}+\sum_{w \in \mathbb{F}_{q^{2}}} \phi(w) \psi^{q}(w) \overline{\theta^{q}(w)} \\
& +\sum_{w \in \mathbb{F}_{q^{2}}^{*}} \phi^{q}(w) \psi^{q}(w) \overline{\theta(w)}+\sum_{w \in \mathbb{F}_{q^{2}}^{*}} \phi^{q}(w) \psi(w) \overline{\theta(w)}+\sum_{w \in \mathbb{F}_{q^{2}}^{*}} \phi^{q}(w) \psi(w) \overline{\theta^{q}(w)} \\
& \left.+\sum_{w \in \mathbb{F}_{q^{2}}^{*}} \phi^{q}(w) \psi^{q}(w) \overline{\theta^{q}(w)}\right) .
\end{aligned}
$$

We need a compact notation to analyse this sum. For three integers $a, b, c \in\{1, q\}$, we write

$$
\Gamma_{[a, b, c]}=\sum_{w \in \mathbb{F}_{q^{*}}} \phi^{a}(w) \psi^{b}(w) \overline{\theta^{c}(w)}
$$

Now consider the condition

$$
\phi^{a} \psi^{b}=\theta^{c}
$$

which we denote by $C[a, b, c]$. By Lemma 4.6, we know that

$$
\Gamma_{[a, b, c]}= \begin{cases}q^{2}-1, & \text { if } C[a, b, c] \text { holds } \\ 0 & \text { otherwise }\end{cases}
$$

Thus we have eight conditions $C[a, b, c]$ for all possible values of $a, b, c$. However, we have the following pattern of equivalences:

C1. $C[1,1,1] \Longleftrightarrow C[q, q, q]$,

C2. $C[1,1, q] \Longleftrightarrow C[q, q, 1]$,

C3. $C[1, q, 1] \Longleftrightarrow C[q, 1, q]$, 
C4. $C[q, 1,1] \Longleftrightarrow C[1, q, q]$.

This can be seen as follows. For example, suppose $C[1,1, q]$ holds, i.e., $\phi \psi=\theta^{q}$. Then raising this equation to the $q$-th power gives $C[q, q, 1]$, since $\theta^{q^{2}}=\theta$. Similarly, starting from $C[q, q, 1]$ we can deduce $C[1,1, q]$. All other cases are similar. Henceforth we will say that $\mathrm{C} 1$ holds if both the corresponding equivalent conditions hold, and similarly for $\mathrm{C} 2, \mathrm{C} 3, \mathrm{C} 4$.

Notice also that at most one of the conditions $\mathrm{C} 1, \ldots, \mathrm{C} 4$ can hold. For example, suppose $\mathrm{C} 2, \mathrm{C} 3$ both hold. Then

$$
\phi^{q} \psi^{q}=\theta \text { and } \phi \psi^{q}=\theta \Longrightarrow \phi=\phi^{q},
$$

which is disallowed. Now consider the additional condition

C0. $\hat{\phi} \hat{\psi}=\hat{\theta}$.

If any of the conditions $\mathrm{C} 1, \ldots, \mathrm{C} 4$ holds, then $\mathrm{C} 0$ also holds. For example, assume $\phi^{q} \psi^{q}=\theta$. Then restricting this equality to $\mathbb{F}_{q}^{*}$ gives $\hat{\phi}^{q} \hat{\psi}^{q}=\hat{\theta}$. Since every character $\alpha$ of $\mathbb{F}_{q}^{*}$ satisfies $\alpha^{q}=\alpha$, we get $\mathrm{C} 0$. The other cases are similar.

Now observe that

$$
\mathbb{S}=\frac{1}{q-1} \sum_{x \in \mathbb{F}_{q}^{*}} \phi(x) \psi(x) \overline{\theta(x)}-\frac{1}{2\left(q^{2}-1\right)} \sum_{[a, b, c]} \Gamma_{[a, b, c]},
$$

where the second summation is over all the eight choices of $[a, b, c]$.

- If none of the conditions $\mathrm{C} 0, \ldots, \mathrm{C} 4$ holds, then $\mathbb{S}=0$.

- If any of the conditions $\mathrm{C} 1, \ldots, \mathrm{C} 4$ holds, then we get

$$
\mathbb{S}=\frac{q-1}{q-1}-\frac{2\left(q^{2}-1\right)}{2\left(q^{2}-1\right)}=0
$$


- If $\mathrm{C} 0$ holds and none of the conditions $\mathrm{C} 1, \ldots, \mathrm{C} 4$ holds, then $\mathbb{S}=1$. These are precisely the characters $\theta$ which give one copy of $X_{\theta}$ in the decomposition.

This completes the calculation for the case $X_{\phi} \otimes X_{\psi}$. We summarize the results in the following proposition.

Proposition 5.13. When $\hat{\phi} \hat{\psi}$ is a non-square, then we have a decomposition

$$
X_{\phi} \otimes X_{\psi} \cong\left(\bigoplus_{\alpha} W_{\alpha, \hat{\phi} \hat{\psi} \alpha^{-1}}\right) \oplus\left(\bigoplus_{\theta \in Q} X_{\theta}\right)
$$

where $Q$ is the set of characters $\theta$ of $\mathbb{F}_{q^{2}}^{*}$ such that $\hat{\phi} \hat{\psi}=\hat{\theta}$, but

$$
\phi \psi \neq \theta, \theta^{q}, \quad \phi \psi^{q} \neq \theta, \theta^{q}
$$

As usual, we make the convention that $X_{\theta}$ and $X_{\theta^{q}}$ represent the same summand.

When $\hat{\phi} \hat{\psi}$ is a square, then we have a decomposition

$$
X_{\phi} \otimes X_{\psi} \cong\left(\bigoplus_{\alpha} W_{\alpha, \hat{\phi} \hat{\psi} \alpha^{-1}}\right) \oplus\left(\bigoplus_{\theta \in Q} X_{\theta}\right) \oplus\left(\bigoplus_{\eta} V_{\eta}\right) \oplus\left(\bigoplus_{\alpha \in Q^{\prime}} U_{\alpha}\right)
$$

where $Q$ has the same meaning as above, and in the first summand those $\alpha$ such that $\alpha^{2}=\hat{\phi} \hat{\psi}$ are disallowed. The third summand is over all characters $\eta$ of $\mathbb{F}_{q}^{*}$ such that $\eta^{2}=\hat{\phi} \hat{\psi}$. Moreover, $Q^{\prime}$ is the set of characters $\alpha$ of $\mathbb{F}_{q}^{*}$ such that $\widetilde{\alpha}=\phi \psi$ or $\phi \psi^{q}$.

We will not state such a proposition explicitly for the last calculation, since the statements tend to become unwieldy. However, the summands in the tensor product are explicitly described via Lemmas 5.2 and 5.3 . 


\subsection{The Decomposition of $W_{\alpha, 1} \otimes W_{\beta, 1}$}

In this case, $\alpha$ and $\beta$ are non-trivial (not necessarily distinct) characters of $\mathbb{F}_{q}^{*}$. The character table is as follows:

\begin{tabular}{|c||c|c|c|c|}
\hline & $a_{x}$ & $b_{x}$ & $c_{x, y}$ & $d_{x, y}$ \\
\hline \hline$\chi_{W_{\alpha, 1} \otimes W_{\beta, 1}}$ & $(q+1)^{2} \alpha(x) \beta(x)$ & $\alpha(x) \beta(x)$ & $(\alpha(x)+\alpha(y))(\beta(x)+\beta(y))$ & 0 \\
\hline
\end{tabular}

Using Lemmas 5.2 and 5.3 we deduce the following statements:

- Let $\gamma$ be a character of $\mathbb{F}_{q}^{*}$. We have

$$
m\left(U_{\gamma}, W_{\alpha, 1} \otimes W_{\beta, 1}\right)=m\left(W_{\alpha^{-1}, 1}, W_{\beta \gamma^{-1}, \gamma^{-1}}\right)
$$

This number is non-zero exactly when the two $W$-type representations on the right are isomorphic, and then it is $=1$. They are isomorphic in either of the following cases:

$$
\begin{aligned}
& \circ \alpha^{-1}=\beta \gamma^{-1} \text { and } 1=\gamma^{-1} \text {, i.e., } \gamma=1 \text { and } \alpha^{-1}=\beta \text {. } \\
& \circ \alpha^{-1}=\gamma^{-1} \text { and } \beta \gamma^{-1}=1 \text {, i.e., } \alpha=\beta=\gamma
\end{aligned}
$$

- We have

$$
m\left(V_{\gamma}, W_{\alpha, 1} \otimes W_{\beta, 1}\right)=m\left(W_{\beta^{-1} \gamma, \gamma}, V \otimes W_{\alpha, 1}\right) .
$$

The last number is known by the calculations in Section 5.4 .

- We have

$$
m\left(X_{\phi}, W_{\alpha, 1} \otimes W_{\beta, 1}\right)=m\left(W_{\beta^{-1}, 1}, W_{\alpha, 1} \otimes X_{\phi^{-1}}\right) .
$$

The last number is known by the calculations in Section 5.6. 


\section{8 .1}

Now we complete the last remaining calculation, i.e., the number of copies of $W_{\gamma, \delta}$ in $W_{\alpha, 1} \otimes W_{\beta, 1}$, where $\gamma \neq \delta$. We have

$$
\begin{aligned}
\mathbb{S} & =\left\langle\chi_{W_{\alpha, 1} \otimes W_{\beta, 1}}, \chi_{W_{\gamma, \delta}}\right\rangle \\
= & \frac{1}{|G|}\left[\sum_{a_{x}}(q+1)^{3} \alpha(x) \beta(x) \overline{\gamma(x) \delta(x)}+\sum_{b_{x}} \alpha(x) \beta(x) \overline{\gamma(x) \delta(x)}\left(q^{2}-1\right)\right. \\
& \left.+\frac{1}{2} \sum_{c_{x, y}}(\alpha(x)+\alpha(y))(\beta(x)+\beta(y)) \overline{(\gamma(x) \delta(y)+\delta(x) \gamma(y)} \cdot\left(q^{2}+q\right)\right] \\
= & \frac{1}{|G|}\left[(q+1)\left(q^{2}+3 q\right) \sum_{x \in \mathbb{F}_{q}^{*}} \alpha(x) \beta(x) \overline{\gamma(x) \delta(x)}+\frac{\left(q^{2}+q\right)}{2}\left(\sum_{c_{x, y}} \alpha(x) \beta(x) \overline{\gamma(x) \delta(y)}\right.\right. \\
& +\alpha(y) \beta(y) \overline{\gamma(x) \delta(y)}+\alpha(x) \beta(y) \overline{\gamma(x) \delta(y)}+\alpha(y) \beta(x) \overline{\gamma(x) \delta(y)}+\alpha(x) \beta(x) \overline{\gamma(y) \delta(x)} \\
& +\alpha(y) \beta(y) \overline{\gamma(y) \delta(x)}+\alpha(x) \beta(y) \overline{\gamma(y) \delta(x)}+\alpha(y) \beta(x) \overline{\gamma(y) \delta(x)})] .
\end{aligned}
$$

This expression reduces to

$$
\begin{aligned}
\mathbb{S} & =\frac{1}{(q-1)} \underbrace{\sum_{x \in \mathbb{F}_{q}^{*}} \alpha \beta \overline{\gamma \delta}(x)}_{S_{0}}+\frac{1}{(q-1)^{2}}[\underbrace{\left(\sum_{x \in \mathbb{F}_{q}^{*}} \alpha \beta \bar{\gamma}(x)\right)\left(\sum_{y \in \mathbb{F}_{q}^{*}} \bar{\delta}(y)\right)}_{S_{1}} \\
+ & \underbrace{\left(\sum_{x \in \mathbb{F}_{q}^{*}} \alpha \beta \bar{\delta}(x)\right)\left(\sum_{y \in \mathbb{F}_{q}^{*}} \bar{\gamma}(y)\right)}_{S_{2}}+\underbrace{\left(\sum_{x \in \mathbb{F}_{q}^{*}} \alpha \bar{\gamma}(x)\right)\left(\sum_{y \in \mathbb{F}_{q}^{*}} \beta \bar{\delta}(y)\right)}_{S_{3}} \\
+ & \underbrace{\left(\sum_{x \in \mathbb{F}_{q}^{*}} \beta \bar{\gamma}(x)\right)\left(\sum_{y \in \mathbb{F}_{q}^{*}} \alpha \bar{\delta}(y)\right)}_{S_{4}} .
\end{aligned}
$$

We need to find out when the expressions $S_{i}$ are non-zero. Consider the following conditions:

C0. $\alpha \beta=\gamma \delta$,

C1. $\alpha \beta=\gamma$ and $\delta=1$, 
C2. $\alpha \beta=\delta$ and $\gamma=1$,

C3. $\alpha=\gamma$ and $\beta=\delta$,

C4. $\beta=\gamma$ and $\alpha=\delta$.

By Lemma 4.5, we know that $S_{0}=q-1$ if $\mathrm{C} 0$ is satisfied, and $S_{0}=0$ otherwise. For $i=1,2,3,4$, we have $S_{i}=(q-1)^{2}$ if $\mathrm{Ci}$ is satisfied and $S_{i}=0$ otherwise.

Notice that at most one of the conditions from C1, C2, C3, C4 can hold simultaneously. For example, if $\mathrm{C} 1$ and $\mathrm{C} 4$ were both true, then we would have $\alpha=1$ which is impossible. The other cases are similar. Moreover, any of the conditions from C1, C2, C3, C4 implies C0. Thus we get the following possibilities:

- If none of the conditions from $\mathrm{C} 0, \ldots, \mathrm{C} 4$ holds, then $\mathbb{S}=0$.

- If $\mathrm{C} 1$ holds, then $\mathbb{S}=2$ and $\{\gamma, \delta\}=\{\alpha \beta, 1\}$. The same conclusion follows if $\mathrm{C} 2$ holds. This case is impossible if $\alpha \beta=1$, i.e., $\alpha=\beta^{-1}$.

- If C3 holds, then $\mathbb{S}=2$ and $\{\gamma, \delta\}=\{\alpha, \beta\}$. The same conclusion follows if $\mathrm{C} 4$ holds. This case is impossible if $\alpha=\beta$.

- If $\mathrm{C} 0$ alone holds, then $\mathbb{S}=1$ and $\{\gamma, \delta\}=\left\{\gamma, \alpha \beta \gamma^{-1}\right\}$ for some $\gamma \neq 1, \alpha, \beta, \alpha \beta$. Moreover, if $\alpha \beta$ is a square, then those $\gamma$ such that $\alpha \beta \gamma^{-1}=\gamma$ (i.e., $\gamma^{2}=\alpha \beta$ ) are also disallowed.

Now consider the following conditions:

D1. $\alpha=\beta^{-1}$,

D2. $\alpha=\beta$,

D3. $\alpha \beta$ is a square, say $\alpha \beta=\mu^{2}$ for some character $\mu$. 
If either D1 or D2 holds, then D3 also holds.

From this analysis, we conclude that the $W$-type summands in $W_{\alpha, 1} \otimes W_{\beta, 1}$ are as follows:

- If D1, D2 both hold, then we get one copy each of $W_{\gamma, \gamma^{-1}}$ for $\gamma \neq 1, \alpha$.

- If D1 (but not D2) holds, then we have two copies of $W_{\alpha, \alpha^{-1}}$ and one copy each of $W_{\gamma, \gamma^{-1}}$ for $\gamma \neq 1, \alpha, \alpha^{-1}$.

- If D2 (but not D1) holds, then we have two copies of $W_{\alpha^{2}, 1}$ and one copy each of $W_{\gamma, \alpha^{2} \gamma^{-1}}$ for $\gamma \neq 1, \alpha, \alpha^{2}$.

- If D3 (but not D1, D2) holds, then we have two copies each of $W_{\alpha, \beta}$ and $W_{\alpha \beta, 1}$, and one copy each of $W_{\gamma, \alpha \beta \gamma^{-1}}$ for $\gamma \neq 1, \alpha, \beta, \mu, \alpha \beta$.

- If none of the Di holds, then we have two copies each of $W_{\alpha, \beta}$ and $W_{\alpha \beta, 1}$, and one copy each of $W_{\gamma, \alpha \beta \gamma^{-1}}$ for $\gamma \neq 1, \alpha, \beta, \alpha \beta$.

This calculation allows us to decompose any representation of the form

$$
W_{\alpha_{1}, \alpha_{2}} \otimes W_{\beta_{1}, \beta_{2}} \cong\left(W_{\alpha_{1} \alpha_{2}^{-1}, 1} \otimes W_{\beta_{1} \beta_{2}^{-1}, 1}\right) \otimes U_{\alpha_{2} \beta_{2}}
$$

At this point, we have completed the analysis of all Clebsch-Gordan decompositions for the group $\operatorname{GL}\left(2, \mathbb{F}_{q}\right)$. 


\section{Bibliography}

[1] Jonathan L. Alperin and Rowen B. Bell, Groups and Representations, Springer, 1995.

[2] Charles W. Curtis and Irving Reiner, Representation Theory of Finite Groups and Associative Algebras, AMS Chelsea Publishing, American Mathematical Society reprint, 2006.

[3] David S. Dummit and Richard M. Foote, Abstract Algebra, Prentice-Hall, 1991.

[4] William Fulton and Joe Harris, Representation Theory, A First Course, Springer-Verlag, New York, 1991.

[5] Joseph A. Gallian, Contemporary Abstract Algebra, Fourth Edition, Narosa Publishing House, 1998.

[6] Kenneth Hoffman and Ray Kunze, Linear Algebra, Second Edition, Prentice Hall, 1971.

[7] Gordon James and Martin Liebeck, Representations and Characters of Groups, Cambridge University Press, 2001.

[8] Dudley E. Littlewood, The Theory of Group Characters and Matrix Representations of Groups, Clarendon Press, Oxford, 1940.

[9] Berndt Sturmfels, Algorithms in Invariant Theory, Springer-Verlag, 1993.

[10] Constantin Teleman, Notes on Representation Theory. Available at https://math.berkeley.edu/ teleman/math/RepThry.pdf (accessed on September 28, 2020). 